\title{
THE SUMMER MEETING IN EAST LANSING
}

The fifty-seventh Summer Meeting and thirty-third Colloquium of the American Mathematical Society were held at Michigan State College, East Lansing, Michigan, Tuesday to Friday, September 2-5, in conjunction with meetings of the Mathematical Association of America, The Institute of Mathematical Statistics, and the Econometric Society.

Over 600 people registered for the meeting, including the following 409 members of the Society:

C. R. Adams, R. B. Adams, G. E. Albert, M. W. Al-Dhahir, B. E. Allen, E. B. Allen, W. R. Allen, C. B. Allendoerfer, A. G. Anderson, R. D. Anderson, T. W. Anderson, R. V. Andree, J. J. Andrews, H. A. Antosiewicz, B. M. Armstrong, K. J. Arnold, Nachmann Aronszajn, M. C. Ayer, W. L. Ayres, H. M. Bacon, I. A. Barnett, D. Y. Barrer, R. C. Bartels, P. T. Bateman, W. F. Bauer, H. W. Becker, E. M. Beesley, E. G. Begle, J. H. Bell, Arthur Bernhart, Benjamin Bernholtz, D. L. Bernstein, W. A. Beyer, R. H. Bing, D. W. Blackett, David Blackwell, H. D. Block, I. M. Blyth, R. P. Boas, H. F. Bohnenblust, R. C. Bose, J. W. Bower, Barron Brainerd, A. T. Brauer, J. L. Brenner, Leonard Bristow, J. C. Brixey, F. E. Browder, M. C. Brown, R. H. Bruck, C. C. Buck, R. C. Buck, C. E. Buell, C. E. Burgess, J. H. Bushey, H. E. Campbell, K. H. Carlson, J. W. Carr, R. E. Carr, E. D. Cashwell, Lamberto Cesari, Abraham Charnes, Y. W. Chen, S. S. Chern, Joshua Chover, A. B. Clarke, F. M. Clarke, H. E. Clarkson, Nathaniel Coburn, D. E. Coffey, Harvey Cohn, R. H. Cole, E. G. H. Comfort, N. B. Conkwright, Geraldine Coon, T. F. Cope, A. H. Copeland, Max Coral, R. R. Coveyou, V. F. Cowling, H. S. M. Coxeter, J. W. Coy, C. C. Craig, M. V. Cross, A. B. Cunningham, D. A. Darling, Robert Davies, W. M. Davis, G. P. Dinneen, W. J. Dixon, J. M. Dobbie, C. L. Dolph, W. F. Donoghue, J. L. Doob, A. C. Downing, D. W. Dubois, R. J. Duffin, W. L. Duren, Ben Dushnik, P. S. Dwyer, J. M. Earl, W. F. Eberlein, J. G. Elliott, D. O. Ellis, Benjamin Epstein, D. H. Erkiletian, M. E. Estill, H. P. Evans, W. H. Fagerstrom, D. T. Finkbeiner, C. D. Firestone, W. H. Fleming, M. M. Flood, K. W. Folley, M. K. Fort, J. S. Frame, Evelyn Frank, Bernard Friedman, W. B. Fulks, R. E. Fullerton, H. K. Fulmer, I. S. Gál, H. L. Garabedian, H. M. Gehman, J. J. Gergen, Murray Gerstenhaber, Leonard Gillman, M. A. Girschick, Wallace Givens, Casper Goffman, S. I. Goldberg, R. A. Good, A. W. Goodman, Lillian Gough, S. H. Gould, Cornelius Gouwens, A. A. Grau, L. M. Graves, R. E. Graves, R. L. Graves, F. L. Griffin, H. C. Griffith, R. K. Haddad, Franklin Haimo, P. R. Halmos, P. C. Hammer, H. A. Hanson, Frank Harary, T. E. Harris, O. G. Harrold, G. E. Hay, R. M. Hayes, L. J. Heider, R. G. Helsel, M. S. Hendrickson, A. S. Henriques, I. N. Herstein, Fritz Herzog, G. W. Hess, D. G. Higman, T. H. Hildebrandt, J. G. Hocking, J. L. Hodges, J. E. Hoffman, R. V. Hogg, F. E. Hohn, D. L. Holl, T. C. Holyoke, Harold Hotelling, Ralph Hull, C. C. Hurd, J. W. Hurst, W. R. Hutcherson, C. G. Jaeger, R. D. James, T. A. Jeeves, W. E. Jenner, L. W. Johnson, R. E. Johnson, B. W. Jones, P. S. Jones, Joachim Kaiser, Shizuo Kakutani, Jan Kalicki, Wilfred Kaplan, Leo Katz, D. E. Kearney, W. H. Keen, M. W. Keller, L. M. Kelly, S. H. Khamis, D. E. Kibbey, W. M. Kincaid, J. M. Kingston, Evelyn Kinney, S. C. Kleene, Erwin Kleinfeld, L. A. Knowler, F. W. Kokomoor, T. C. Koopmans, H. W. Kuhn, E. G. Kundert, 
R. M. Lakness, H. G. Landau, R. E. Langer, Leo Lapidus, E. H. Larguier, H. D. Larsen, C. G. Latimer, L. M. LeCam, J. R. Lee, G. F. Leger, E. L. Lehmann, Walter Leighton, Frank Levin, D. J. Lewis, F. A. Lewis, H. M. Lieberstein, A. J. Lohwater, C. I. Lubin, J. M. McArtney, Dorothy McCoy, N. H. McCoy, R. W. MacDowell, C. C. MacDuffee, G. W. Mackey, Saunders MacLane, H. M. MacNeille, H. B. Mann, E. C. Marth, W. T. Martin, Imanuel Marx, F. J. Massey, K. O. May, J. P. Mayberry, L. E. Mehlenbacher, C. W. Mendel, B. E. Meserve, D. M. Mesner, E. J. Mickle, W. E. Milne, Hazleton Mirkil, Benjamin Ernest Mitchell, Josephine Mitchell, Don Mittleman, E. E. Moise, H. F. Montague, M. D. Montgomery, J. C. Moore, W. K. Moore, D. C. Morrow, Frederick Mosteller, T. S. Motzkin, E. J. Moulton, S. T. C. Moy, H. T. Muhly, M. E. Munroe, C. W. Munshower, W. L. Murdock, G. G. Murray, S. B. Myers, Zeev Nehari, A. L. Nelson, W. J. Nemerever, C. J. Nesbitt, Jerzy Neyman, Ivan Niven, E. A. Nordhaus, E. S. Northam, Ilse Novak, R. E. O’Donnell, E. G. Olds, Ingram Olkin, Richard Otter, J. C. Oxtoby, N. H. Painter, O. O. Pardee, Emanuel Parzen, M. H. Payne, L. L. Pennisi, F. W. Perkins, H. P. Pettit, George Piranian, Everett Pitcher, J. C. Polley, Pasquale Porcelli, J. E. Powell, G. B. Price, G. C. E. Prins, L. E. Pursell, Gustave Rabson, C. B. Rader, Tibor Rad6, J. F. Randolph, G. E. Raynor, M. O. Reade, Mina Rees, Paul Reichelderfer, W. T. Reid, H. J. Reiter, R. R. Reynolds, H. B. Ribeiro, H. G. Rice, C. E. Rickart, F. A. Rickey, P. R. Rider, F. D. Rigby, J. H. Roberts, R. A. Rosenbaum, Murray Rosenblatt, P. C. Rosenbloom, Arthur Rosenthal, M. F. Rosskopf, E. H. Rothe, Herman Rubin, Herbert Ruderfer, Walter Rudin, Charles Saltzer, Hans Samelson, James Sanders, I. R. Savage, A. C. Schaeffer, R. D. Schafer, A. T. Schafer, J. A. Schatz, Henry Scheffe, E. V. Schenkman, E. R. Schneckenburger, Lowell Schoenfeld, Augusta Schurrer, C. H. W. Sedgewick, I. E, Segal, Esther Seiden, D. B. Shaffer, D. H. Shaffer, M. E. Shanks, L. S. Shapley, Seymour Sherman, R. L. Shively, Edward Silverman, M. L. Slater, M. F. Smiley, G. W. Smith, S. S. Smith, V. E. Smith, Ernst Snapper, H. H. Snyder, W. S. Snyder, E. S. Sokolnikoff, D. E. Spencer, V. E. Spencer, C. E. Springer, E. P. Starke, F. H. Steen, Arthur Stein, H. B. Stelson, C. F. Stephens, B. M. Stewart, R. W. Stokes, R. R. Stoll, M. H. Stone, D. D. Strebe, P. C. Sweetland, Gabor Szegö, Alfred Tarski, M. E. Taylor, W. C. Taylor, H. P. Thielman, W. J. Thron, F. H. Tingey, H. E. Tinnappel, E. W. Titt, C. J. Titus, John Todd, Leonard Tornheim, E. A. Trabant, C. K. Tsao, A. W. Tucker, Bryant Tuckerman, J. W. Tukey, W. T. Tutte, Eugene Usdin, E. P. Vance, N. H. Vaughan, D. F. Votaw, L. I. Wade, G. W. Walker, R. M. Walter, S. S. Walters, D. L. Webb, G. C. Webber, C. P. Wells, I. L. Wente, John Wermer, G. W. Whitehead, W. F. Whitmore, W. M. Whyburn, L. R. Wilcox, J. D. Wilkes, J. E. Wilkins, G. M. Wing, Y. K. Wong, M. A. Woodbury, Oswald Wyler, L. C. Young, Daniel Zelinsky, J. L. Zemmer, J. A. Zilber, J. W. Zimmer.

The Colloquium Lectures, on Arithmetical classes and types of algebraic systems, were presented by Professor Alfred Tarski of the University of California on Tuesday afternoon and Wednesday, Thursday, and Friday mornings. Presiding officers were, in order, Professors S. C. Kleene, C. C. MacDuffee, C. B. Allendoerfer, and R. C. Buck.

The Committee to Select Hour Speakers for Annual and Summer Meetings invited one speaker. On Thursday, September 4, Professor E. E. Moise of the University of Michigan gave an address entitled 
On the topology of 3-manifolds. Professor R. H. Bing presided.

The organized social events provided for the Society consisted of: an informal coffee hour on Monday evening in the Conference Room of the Physics-Mathematics Building, an informal tea on Tuesday afternoon in Phillips Lower Lounge, trips to the Oldsmobile plant in Lansing on Wednesday and Thursday, and a trip to Henry Ford Museum and Greenfield Village on Thursday. A banquet was held on Wednesday evening in the Union Building. Professor C. B. Allendoerfer, of the University of Washington, was toastmaster and the main speaker of the evening was President John A. Hannah of Michigan State College. A concert by members of the Department of Music of Michigan State College was given on Thursday evening in the Music Building, followed by a party sponsored by the Institute of Mathematical Statistics.

Activities for women included campus tours on Tuesday and Wednesday.

The Council met on Tuesday evening, September 2.

The Secretary announced the election of the following fifty-three persons to ordinary membership in the Society:

Mr. Brian Abrahamson, Graduate Student, University of Chicago;

Mr. Maurice J. Aegerter, Student, University of Wisconsin;

Mr. George Bachman, New York University;

Mr. William Aaron Beyer, Graduate Assistant, Pennsylvania State College;

Dr. Victor Wayne Bolie, Mathematician, Collins Radio Company, Cedar Rapids, Iowa;

Mr. Carmen Nicholas Campopiano, Teaching Assistant, Rutgers University;

Mr. Stanley Howard Cohn, Instructor, Fournier Institute of Technology, Lemont, Illinois;

Mr. Pierre Euclide Conner, Jr., Tulane University;

Mr. Walter Cornetz, Communications Engineer, Grumman Aircraft Engineering Corporation, Bethpage, Long Island, New York;

Dr. Charles Raymond DeCarlo, Applied Science Representative, International Business Machines Corporation, Pittsburgh, Pennsylvania;

Sister Mary Perpetual Help Dowling, Incarnate Word College, San Antonio, Texas; Associate Professor Marcellus M. Dreiling, St. Joseph's College, Collegeville, Indiana; Mr. Frederick Robert Eisler, Electronics Engineer, Western Electric Company, Jersey City, New Jersey;

Mr. David Israel Epstein, New York University;

Mr. Sidney Marshall Foulks, Research Physicist, Magnolia Petroleum Company, Dallas, Texas;

Mr. William Franklin Furr, Instructor, Southern University, Baton Rouge, Louisiana;

Dr. R. Wayne Gardner, Olivet Nazarene College, Kankakee, Illinois;

Mr. Herbert B. Goertzel, Mathematics Assistant, Oak Ridge National Laboratory, Oak Ridge, Tennessee;

Dr. Richard Pettengill Gosselin, Mathematician, Institute for Air Weapons Research, University of Chicago;

Mr. Edward Everett Grace, Teaching Fellow, University of North Carolina; 
Assistant Professor Alfred Thomas Hind, Jr., Clemson College;

Mr. John Edward Hoffman, Graduate Assistant, University of Oklahoma;

Mr. Mark Holzman, Research Engineer, Western Geophysical Company, Los Angeles, California;

Mr. William James Hood, Graduate Student, Stanford University;

Mr. Jiro Ishihara, Digital Computer Laboratory, Massachusetts Institute of Technology;

Mr. Ted R. Jenkins, Teaching Fellow, University of Washington;

Mr. Raghunath Prasad Kanoria, Research Student, Calcutta University, Calcutta, India;

Mr. Frank Kozin, Illinois Institute of Technology;

Professor Arwid Ottomar Count Kumin-Kumins, Annhurst College, South Woodstock, Connecticut;

Mr. R. Sherman Lehman, Research Assistant, Stanford University;

Mr. Hiramie Theodore McAdams, Research Chemist, Aluminum Research Laboratories, East St. Louis, Illinois;

Mr. Paul Brainerd McKowen, Aerodynamicist, Bell Aircraft Corporation, Buffalo 5, New York;

Mr. William Harold Mays, Instructor, Pikeville College, Pikeville, Kentucky;

Mr. Norman Wilfred Moir, Jr., Assistant Instructor, Temple University;

Mr. James C. Morelock, Graduate Fellow, University of Florida;

Assistant Professor Vernon Howard Morrill, Hardin-Simmons University, Abilene, Texas;

Dr. William Nachbar, Applied Mathematician, Boeing Airplane Company, Seattle, Washington;

Mr. Louis Legendre Pennisi, Instructor, University of Illinois;

Mr. William James Perry, Instructor, Pennsylvania State College;

Mr. Philip C. Rapp, Supervisor, Analysis Section, Bell Aircraft Corporation, Buffalo 5, New York;

Assistant Professor Dolores Agnes Rickens, College of St. Elizabeth, Convent Station, New Jersey;

Mr. John Theodore Robacker, Mathematician, Northrop Aircraft Company, Los Angeles, California;

Mr. Albert S. Rosenthal, Mathematician, Naval Air Development Center, Johnsville, Pennsylvania;

Dr. William Keyser Saunders, Mathematician, National Bureau of Standards, Washington, D. C.;

Mr. Henry Sharp, Duke University;

Mr. Allen Lowell Shields, Fellow, Massachusetts Institute of Technology;

Mr. Robert Simon, Senior Project Analytical Engineer-Mathematical Consultant, Chance Vought Aircraft, Dallas, Texas;

Mr. David Slepian, Research Mathematician, Bell Telephone Laboratories, Murray Hill, New Jersey;

Mr. Bernard Sohmer, New York University;

Dr. David D. Strebe, Instructor, University of Buffalo;

Mr. Alfred L. Toth, Instructor, United States Military Academy;

Miss Ruth Ann Wagner, Mathematician, The Rand Corporation, Santa Monica, California;

Mr. Michael Yanowitch, Mathematician, Reeves Instrument Corporation, New York, New York. 
It was reported that the following person had been elected as a nominee of the institution as indicated:

Kenyon College: Mr. Roger Harold Geeslin.

The Secretary announced that the following person had been admitted to the Society in accordance with a reciprocity agreement with the Société Mathématique de France: Professor Robert Faure, University of Hanoi, Tonkin, French Indo China. Palais Bella Bista, Square D'Oftende, Nice, France.

The University of California, Davis, California, DePaul University, Chicago, Illinois, and the University of New Hampshire, Durham, New Hampshire, were elected to institutional membership.

The following appointments of representatives of the Society were reported: Professor J. R. Kline at the inauguration of Lewis Webster Jones as President of Rutgers University on May 8, 1952; Professor E. G. Begle at the convocation ceremonies at the United States Military Academy celebrating the Sesquicentennial year of the Academy on May 20, 1952 ; Professor M. H. Martin as the Society's representative at the National Congress of Applied Mechanics in Istanbul on August 20-28, 1952.

The following additional appointments by the President were reported: as a Committee on a Summer Mathematical Institute: A. A. Albert (Chairman), Samuel Eilenberg, Saunders MacLane, D. C. Spencer, and Oscar Zariski; as a committee to study problems of nominations by petition: William Feller (Chairman), Warren Ambrose, J. W. Green, G. A. Hedlund, M. R. Hestenes, and S. B. Myers; as a committee on arrangements for the Annual Meeting to be held at Washington University on December 27-29, 1952: R. R. Middlemiss (Chairman), T. L. Downs, H. Margaret Elliott, H. M. Gehman, Francis Regan, Marlow Sholander, and J. W. T. Youngs; Professor M. H. Martin appointed a representative to the U. S. National Committee on Theoretical and Applied Mechanics for a four-year term beginning January 1, 1953; Professor R. V. Churchill re-appointed a member of the Editorial Committee on Applied Mathematics Symposium Proceedings for a three-year term beginning July 1, 1952 (Committee now consists of: Eric Reissner (Chairman), A. H. Taub, and R. V. Churchill); Professor Eric Reissner re-appointed as Chairman of the Editorial Committee on Applied Mathematics Symposium Proceedings for the period July 1, 1952 to June 30, 1953; Professors B. P. Gill and C. H. W. Sedgewick as auditors for 1952.

The following dates of meetings have been approved by the Council: April 24-25, 1953, at the University of Chicago and May 2, 
1953, at Stanford University. The Council voted to accept an invitation to hold the Sixth Applied Mathematics Symposium at the Corona Laboratories of the National Bureau of Standards on August 26-28, 1953. The topic of this Symposium will be Numerical Analysis.

An invitation to hold the Summer Meeting for 1954 at the University of Wyoming, Laramie, Wyoming, was accepted.

Invitations to give addresses in 1952 were announced: N. Aronszajn, November meeting at Lafayette; H. H. Goldstine, November meeting at Raleigh; and A. M. Gleason, Annual Meeting at St. Louis.

The Secretary reported that the Visiting Lecturer of the Society, Professor Marshall H. Stone of the University of Chicago, had completed his program of lectures. During the academic year 1951-52, Professor Stone visited almost all parts of the country, traveling over 30,000 miles, and delivered a total of 81 lectures.

The Executive Director reported that a combined directory of the membership of the Society and of the Mathematical Association of America is under preparation. This directory will be sent to members of the Society one year and the Association the next year. The directory will be a separate publication and will not be bound with the Bulletin.

The Council voted to have Society membership cards made up. These will be issued to members of the Society on request. Requests for these cards should be sent to the Headquarters of the Society.

The Council voted to recommend to the Trustees that the Society keep available, by reprinting if necessary, volumes of the Transactions for the previous ten years, that no reprinting of back numbers of the Bulletin be done, but that an attempt be made to keep back volumes of the Bulletin for the previous ten years available by ordering sufficient copies from the printer at the time of publication. Until this time, the Society has kept available all back numbers of both the Transactions and Bulletin. This has necessitated reprinting numerous older numbers and has tied up a large part of the Society's funds.

The Council voted to approve the following resolution, similar to one approved by the Mathematical Association of America.

The Council of the American Mathematical Society has voted to instruct the Secretary to send copies of the following resolution to the Governor of the State of Oklahoma, to the presiding officers of the two houses of the Oklahoma Legislature, to the Attorney General of Oklahoma, and to the Presidents of Oklahoma A. \& M. College and the University of Oklahoma. 
"The Council of the American Mathematical Society has considered the effect upon mathematics and mathematicians of the Oklahoma Loyalty Oath Law of April 9, 1951 (Enrolled House Bill No. 8, 23 Legislature, Laws 1951, 510.S.1941 (Supp.) SS 37.1-37.8).

"Without presuming to censure the State of Oklahoma for this action, the Council wishes to convey to the heads of government in Oklahoma its view that many mathematicians regard this type of legislation as dangerous to individual liberty and to the principles which protect freedom of thought in a university from political infringement, and as ineffective for the purpose of eliminating disloyal influences from the universities. Even those who sign such oaths of ten do so more in a spirit of tolerance for the patriotic motives which conceived the law than in respect for the law itself, or more in fear than in concurrence.

"Whenever such laws are enacted, it is to be expected that at least a few of the American idealists, the sincerely religious people, and the courageous lovers of liberty will rebel, with tragic effects upon themselves, their families, and upon the universities where they work. It is not to be expected that such legislation will be effective in eliminating from the faculties men who are dangerous to the national welfare, so that the injury caused is a useless waste, as at Oklahoma A. \& M. College, where a department of mathematics which had achieved much recognition for its mathematical work was seriously damaged."

A general business meeting of the Society was held on Wednesday, September 3. Professor T. H. Hildebrandt presided. The members present voted to amend the by-laws as follows:

(a) to delete the post of Librarian as specified in Section 1 of Article I, Section 1 of Article VII, and Section 6 of Article VII, and to delete Section 3 of Article I concerning the Society's exchanges.

(b) to amend Article III to create an elected Committee on Printing and Publishing, consisting of three members, and to amend Article XI by adding a Section 3 specifying the duties of this Committee.

(c) to amend Article IX of the by-laws by revising Sections 1, 2, 7, 9, and 11. These revisions eliminate fractional years of membership and also eliminate reduced dues for the early years of membership. Dues bills will now be distributed before the end of the calendar year. The Bulletin and Proceedings will be sent, as privileges of membership, only to those members who have discharged their dues for the year in question.

(d) to eliminate from the by-laws, as of December 31,1952, certain provisions which will have become obsolete as of that time. These are the last sentence of Section 1 of Article IV, and the last three paragraphs of Section 1 of Article VII.

The by-laws of the Society, as revised, appear at the end of this number of the Bulletin.

The Secretary reported that the Council, with the approval of the Board of Trustees, has voted that any member on the rolls of 
the Society in 1952 and entitled to reduced dues under the dues schedule in effect at the beginning of 1952 shall continue to have his dues fixed according to the old schedule rather than the new schedule specified in Section 2 of Article IX.

Abstracts of the papers read follow. Presiding officers at the sessions for contributed papers were Professors W. T. Reid, H. T. Muhly, R. H. Bing, P. R. Halmos, L. R. Wilcox, Dr. Seymour Sherman, Professors W. M. Whyburn, Saunders MacLane, O. G. Harrold, C. E. Rickart, A. H. Copeland, G. W. Whitehead, G. S. Young, W. F. Eberlein, P. C. Rosenbloom, Fritz Herzog, B. M. Stewart, and L. M. Kelly.

Papers whose abstract numbers are followed by the letter " $t$ " were read by title. Paper number 575 was presented by Professor Good, 582 by Professor Kalicki, 584 by Professor Olkin, 598 by Professor Aronszajn, 610 by Professor Dolph, 615 by Dr. Fleming, 626 by Professor Halmos, 628 by Professor Herzog, 629 by Professor Piranian, 663 by Professor Titt, 664 by Dr. Titus, 674 by Mr. Shaffer, 679 by Professor Hohn, 691 by Professor Hutcherson, 694 by Mr. Kelly, 695 by Professor Radó, 698 by Professor Motzkin, 701 by Mr. Suppes, 703 by Professor Rosenblatt, and 726 by Professor Shanks.

Dr. Blair was introduced by Professor J. W. T. Youngs, Mr. Kozin, by Professor Gordon Pall, Mr. Burrill by Professor M. F. Smiley, Mr. Hammersley by Mr. John Todd, Professor Kimball by Professor D. L. Holl, Dr. Pennisi by Professor L. M. Graves, Professor Samuelson by Dr. G. E. Forsythe, Mr. Davis by Professor R. M. Thrall, and Mr. Sharp by Professor J. H. Roberts.

\section{Algebra ANd Theory of Numbers}

557t. Frederick Bagemihl and Paul Erdös: Intersections of prescribed cardinality. Preliminary report.

The authors prove the following theorem, which generalizes a recently published result [F. Bagemihl, Ann. of Math. vol. 55 (1952) pp. 34-37]: Let $\mathfrak{A}$ and $\mathfrak{B}$ be sets, and to every $a \in \mathfrak{P}$ let there correspond a subset, $\Gamma(a)$, of $\mathfrak{B}$. Suppose that the following conditions are satisfied: (I) $|\mathfrak{A}| \leqq \boldsymbol{N}_{\alpha}$; (II) $|\Gamma(a)| \geqq \boldsymbol{N}_{\alpha}$ for every $a \in \mathfrak{R}$; (III) if $a \in \mathfrak{A}$, there is a cardinal number $\mathfrak{n}_{a} \geqq 1$ such that, if $a^{\prime} \in \mathfrak{R}$ and $a^{\prime} \neq a$, then $\left|\Gamma(a) \bigcap \Gamma\left(a^{\prime}\right)\right| \leqq \mathfrak{n}_{a}$; and the complex of cardinal numbers $\mathfrak{n}_{a}(a \in \mathfrak{R})$ is strongly less than $\aleph_{\alpha}$ (i.e., every sum of fewer than $\aleph_{\alpha}$ terms belonging to the complex is less than $\mathcal{N}_{\alpha}$ ); (IV) there is a cardinal number $\mathfrak{m} \geqq 1$ with the following properties: (a) $\delta^{\mathfrak{m}}<\boldsymbol{N}_{\alpha}$ for every $\delta<\boldsymbol{N}_{\alpha}$; (b) if $\mathfrak{B}^{\prime}$ is an arbitrary subset of $\mathfrak{B}$, with $\left|\mathfrak{B}^{\prime}\right|=\mathfrak{m}$, and $\mathfrak{m}\left(\mathfrak{B}^{\prime}\right)$ is the number of elements $a$ of $\mathfrak{A}$ for which $\mathfrak{B}^{\prime}$ is a subset of $\Gamma(a)$, then the complex of cardinal numbers $\mathfrak{m}\left(\mathfrak{B}^{\prime}\right)$, obtained by letting $\mathfrak{B}^{\prime}$ run through all the subsets of $\mathfrak{B}$ having $\mathfrak{m}$ elements, is strongly less than $\aleph_{\alpha}$. With every $a \in \mathfrak{A}$, associate a cardinal number $\mathfrak{a}_{a}$ such that $(\mathrm{V}) \mathfrak{m}+\mathfrak{n}_{a}-1 \leqq \mathfrak{a}_{a} \leqq \boldsymbol{N}_{\alpha}$. Then there exists a subset $\mathfrak{B}^{*}$ of $\mathfrak{B}$, 
with $\left|\mathfrak{B}^{*}\right| \leqq \mathfrak{N}_{\alpha}$, such that $|\mathfrak{B} * \cap \Gamma(a)|=\mathfrak{a}_{a}$ for every $a \in \mathfrak{A}$. An illustration of this theorem is the following: With every straight line $l$ in the plane, associate a cardinal number $\mathfrak{a}_{l}\left(2 \leqq \mathfrak{a}_{l} \leqq \aleph\right)$. Then there exists a point set which is intersected by every line $l$ in precisely $\mathfrak{a}_{l}$ points. (Received May 28, 1952.)

\section{8t. H. W. Becker: Dual Williams vectors (Pythagorean tetra- hedrons).}

In the notation of previous abstracts (Bull. Amer. Math. Soc. (1952)) if $u, v, x, y, z, t$ are each of the form $\sum_{0} q_{m} r^{n-2 m} s^{2 m+p}, p=0$ or 1 , then their dual forms $\sum_{0}(-)^{m} q_{m} r^{n-2 m} s^{2 m+p}$ also define W.V. under some permutation of the six components, such as $(t u x)(v z y)$, or $(t y)(u v)(x z)$. When not self-dual, these W.V. have properties surprisingly different from their duals. A family of W.V. solutions consists in all those derivable from a given parametric solution under $r, s \rightarrow f_{r}(r, s), f_{s}(r, s)$, and their transforms $u, v, x, y, z, t \rightarrow t y, v x, v y, x y, x z, u v$, etc. There are six families whose simplest examples are among the 17 sub-miniature W.V. now known, with $t<10^{4}$. Four families are Carmichael vectors: the Fermat I and II, Dickson's History II, p. 175 and repeatedly thereafter; the Hillyer triad, p. 174 (under $r, s \rightarrow r \pm s$ the Euler triad, p. 474); and the Rolle, p. 447 (using first powers instead of squares of the two parameters) or equivalently, Fermat, p. 172. The other two are the Euler, p. 668 (ii); and Lenhart, pp. 506-507. The Hillyer family is self-dual, in that its members are either self-dual or duals of each other. The Rolle and Euler families are duals, when not self-dual. Dualities exist between members of any pair of the Fermat I and II and Lenhart families. So, new discoveries may be expected as twins. (Received July 21, 1952).

\section{9t. H. W. Becker: The tetradization transform on Carmichael} vectors.

A C.V. depends on solution of $y=4\left(a b c d\left(a^{2}-b^{2}\right)\left(c^{2}-d^{2}\right)\right)^{1 / 2}, t=\left(a^{2}+b^{2}\right)\left(c^{2}+d^{2}\right)$ in integers. A W.V. depends on integer $z=2(i \phi k \lambda)^{1 / 2}, x=\left(\left(i^{2}-\phi^{2}\right)\left(k^{2}-\lambda^{2}\right)\right)^{1 / 2}$. The Petrus transform of a W.V. is a C.V., with $T, U=t^{2} \pm y^{2}, X=v^{2}-u^{2}, V=2 u v, Y=2 x z$, $Z=2 t y, T=\left(A^{2}+B^{2}\right)\left(C^{2}+D^{2}\right), A=i, B=\phi, C=k, D=\lambda$. It follows that if $(a, b, c, d)$ is a C.V., then intermediate between it and its Petrus transform $(A, B, C, D)$ there is a tetrad of C.V.: $(a, b, A, B),(a, b, C, D),(c, d, A, B)$, and $(c, d, C, D)$. Numbering the respective $t$ 's and $y$ 's accordingly, then $t T=t_{1} t_{4}=t_{2} t_{3}, y Y=y_{1} y_{4}=y_{2} y_{3}$. In the Rolle C.V., $a=A=r, b=B=s, \quad c=\left(r^{2}+s^{2}\right)^{2}, \quad d=4 r s\left(r^{2}-s^{2}\right), \quad C=\left(r^{4}+6 r^{2} s^{2}-s^{4}\right)^{2}$, $D=s\left(3 r^{4}-6 r^{2} s^{2}-s^{4}\right)^{2}$, so actually only $(c, d, C, D)$ is interposed. With the Hillyer C.V., only two new C.V. are introduced, the other two being members of the triad. But with Fermat I and II C.V., the tetrad is entirely new and distinct, rounding up into formulation many specimens hitherto mavericks. Miniature C.V. are those with $t<10^{8} ; 43$ examples are tabulated. A complete census depends on, and contributes to, the classification of Pythagorean triangles according to area/ $\square$. Apparently the most populous class is that of 1254 , including the Pythagorean couples 11,$8 ; 19,8$; 22,$3 ; 38,11 ; 147,128 ; 486,475 ; 507,32 ; 1350,19 ; 1862,1859 ; \cdots$ besides $185^{2}$, $1254 \cdot 4^{2}$ etc. on substituting these couples into the Rolle $c, d$ and $C, D$ above. (Received July 21,1952 .)

\section{D. W. Blackett: Simple and semisimple near-rings.}

A "near-ring" is an algebraic system which satisfies all the ring axioms except possibly one of the distributive laws (say the right one) and the commutative law of 
addition. The right ideals of a ring are both the right modules of the ring and the kernels of right homomorphisms of the ring. For a near-ring the right modules are not identical with the kernels of right homomorphisms. These kernels will be called the right ideals of the near-ring. For those near-rings in which the additive identity annihilates the near-ring from the left, every right ideal is also a right module. A nearring is called semisimple if the additive identity annihilates the near-ring from the left, the near-ring contains no nonzero nilpotent right modules, and the right modules satisfy the descending chain condition. Every semisimple near-ring has only a finite number of nonisomorphic types of irreducible right representation spaces. If there is only one such type, the semisimple near-ring is called simple. Every semisimple nearring may be represented uniquely as a direct sum of simple sub-near-rings. Each of these sub-near-rings is the sum of the right modules which are irreducible spaces of a particular type. (Received June 25, 1952.)

\section{R. L. Blair: Stone's topology for a binary relation.}

Let $R^{*}$ be the transitive relation generated by a binary relation $R$ on a set $L$. If $T \subseteq L$, let $T^{-}=\left[x \in L ; x R^{*} T\right]$ and $T^{+}=\left[x \in L ; T R^{*} x\right]$. An element $x \in L$ is s-irreducible (relative to $R$ ) in case $L R^{*} x$ does not hold, and, for every two-element subset $[a, b]$ of $L,[a, b]-\subseteq[x]-$ implies $a R^{*} x$ or $b R^{*} x$. A Stone topology $\gamma(R)$ is introduced in the set $S$ of all $s$-irreducible elements of $L$ by defining the closure of $S_{1} \subseteq S$ to be $S \cap S_{1}{ }^{+}$(cf. M. H. Stone, Trans. Amer. Math. Soc. vol. 41 (1937) pp. 375-481). When $L$ is the set of ideals of a ring and $R$ denotes set inclusion, $\gamma(R)$ contains the spaces of prime (McCoy, Amer. J. Math. vol. 71 (1949) pp. 823-833) and primitive (Jacobson, Proc. Nat. Acad. Sci. U.S.A. vol. 31 (1945) pp. 333-338) ideals as, in general, proper subspaces. Additional applications are made to lattices, including lattices of continuous functions. The preceding observations motivate a study of lattices each of whose elements is a meet of meet-irreducible elements. In this connection the following result is proved: If every maximal dual ideal of a complete complemented modular lattice $L$ is completely prime, then $L$ is atomic. This, incidentally, yields an answer to a question raised by Birkhoff and Frink (Trans. Amer. Math. Soc. vol. 64 (1948) pp. 299-316) concerning a type of representation of a lattice by sets. (Received July 21, 1952.)

\section{2t. Volodymyr Bohun-Chudyniv: On orthogonal and non-} orthogonal closed systems of $K$-nions and their applications.

The aims of this paper are: (1) To define the notion of a closed system of $K$-nions of $2^{k+2}$ rank and $K$ th degree composed of Hamilton's systems of quaternions $\left[1, i_{x}, i_{y}, i_{z}\right]$ and demonstrate a method for determining such systems on the basis of $n=2^{k+2}-1$ imaginary units. (2) To define the notion of the volume of a closed system and to demonstrate a method for determining its dimension. (3) To define the notion of a closed manifold of systems of $2^{k+2}$ rank and $K$ th degree; a method is given for determining its value. (4) To indicate the criteria of the classification of such systems. (5) To give a generalized expression of Euler's formula for square polynomials for an arbitrary $n>4$ (Euler has given a formula for $n=4$ ). (6) To give schemes and the method for determining, with the help of these systems, square matrices composed of integers $\left(2^{2 k+4}\right)$ and $\left(2^{k+2}-1\right)^{2}$. These matrices satisfy all conditions of the Euler problem. L. Euler (Novi Comm. Acad. Petrop. vol. 15 (1770) p. 75; Comm. Arith. vol. I, pp. 427-443) gave only the schemes for $k=0$. Bibliography: (I) Hamilton, Lectures on Quaternions, Dublin, 1853; (II) H. Grassmann, Die lineale Ausdehnungslehre, Leipzig, 1844; (III) Frobenius, Grelli Journ. vol. 84 (1878) p. 59; (IV) Cayley, 
J. Reine Angew. Math. vol. 50, pp. 192, 202; (V) D. A. Grave, Akad. Nauk S.S.S.R. (1938), Trak po Alg. Anal., I, str. 48-57; (VI) V. Bohun-Chudyniv, On a method and general scheme for the solution of the Euler problem (Bull. Amer. Math. Soc. Abstract 58-3-269). (Received July 17, 1952.)

\section{A. T. Brauer: On the least primitive root $(\bmod p)$.}

J. Vinogradoff [Journ. Soc. Phys. Math. de Perm. vol. 1 (1918) pp. 94-98] proved the following theorem by analytic methods. Let $p$ be a prime, $g$ the smallest positive primitive root $\bmod p$, and $k$ the number of different prime divisors of $p-1$, then $g<\left\{2^{k}(p-1) p^{1 / 2} \log p\right\} / \phi(p-1)$. In this paper the following much larger bound is obtained, but by purely elementary methods using a theorem of A. Brauer and T. L. Reynolds [Canadian Journal of Mathematics vol. 3 (1951) pp. 367-374]. If $p$ is a prime of form $4 n+1$ and $r=2^{k}$, then $g<p^{(r-1) / r}$. The same bound holds for the absolute value of the absolute smallest primitive root of primes of form $4 n+3$. (Received September 2, 1952.)

\section{J. L. Brenner: The matrix equation $A X=B$.}

Necessary and sufficient conditions are given for the solvability of the matrix equation $A X=B$. Here $A, B$ are given finite matrices; the elements of $A, B, X$ come from a principal ideal ring $R$, or a ring $S$ of slightly more general nature. The method is to find a complete set of invariants of $\{A X\}$ when $X$ runs through the set of all matrices which have two-sided inverses. This amounts to finding a canonical form for $A$. If the canonical form for $B$ can be obtained from the canonical form for $A$ by replacing some columns of the latter by zero columns, then $A X=B$ is solvable. (Received July $17,1952$.

565. H. E. Campbell: $A$ converse of Cartan's criterion for solvability.

Let $R_{x}$ be the transformation $a \rightarrow a x$ in a Lie algebra $\&$ over a field of characteristic zero. Then Cartan's criterion for solvability is that if the trace $t\left(R_{x}^{2}\right)=0$ for all $x$ of $\mathfrak{R}$, then $\mathfrak{R}$ is solvable. A construction is given to prove that if $\mathfrak{R}$ is solvable over a field $F$ of characteristic zero, then $\mathfrak{N}$ can be imbedded in a Lie algebra $\overline{\mathfrak{N}}$ over a field $\bar{F} \supseteq F$ such that $t\left(R_{x} R_{y}\right)=0$ for all $x, y$ of $\overline{\mathfrak{l}}$. More generally if $\mathfrak{l}=\mathfrak{S}+\mathfrak{N}$ where $\mathfrak{S}$ is semi-simple and $\mathfrak{N}$ is solvable, then $\mathfrak{\ell}$ can be imbedded in $\bar{l}=\mathfrak{S}+\overline{\mathfrak{R}}$ over a field $\bar{F} \supseteq F$ such that $t\left(R_{x} R_{y}\right)=0$ for all $x$ of $\overline{\mathfrak{R}}$ and all $y$ of $\overline{\mathfrak{B}}$. Also any faithful representation $x \rightarrow S_{x}$ of $\mathbb{R}=\mathfrak{S}+\mathfrak{N}$ by matrices can be extended to a faithful representation $x \rightarrow \bar{S}_{x}$

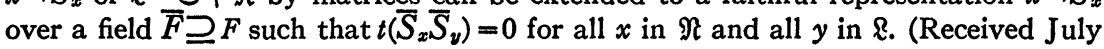
17, 1952.)

566t. Leonard Carlitz: $A$ note on the multiplication formulas for the Bernoulli and Euler polynomials.

As Nielsen has pointed out, if a normalized polynomial of degree $m$ satisfies the multiplication formula for the Bernoulli polynomial for a single value $>1$ of the multiplier, then it is identical with $B_{m}(x)$; a similar result holds for $E_{m}(x)$. In the case of the mixed equation the situation is somewhat different. We show that if normalized polynomials satisfy $g_{m-1}(k x)=-\left(2 k^{m-1} / m\right) \sum_{s-0}^{h-1}(-1)^{8} f_{m}(x+s / k)$ for two distinct even values of $k$, then $f_{m}(x)=B_{m}(x)+a_{m}, g_{m-1}(x)=E_{m-1}(x)$, where $a_{m}$ is an arbitrary constant. A like result holds in the case of the Bernoulli and Euler polynomials of higher order. More generally a result of this sort holds for the Eulerian polynomials 
$\phi_{m}(x, \alpha)$ defined by $\left((1-\alpha) /\left(1-\alpha e^{t}\right)\right) e^{x^{t}}=\sum_{m=0}^{\infty}\left(\phi_{m}(x, \alpha) / m !\right) t^{m}$, where $\alpha \neq 1$ is a root of unity. (Received July 21, 1952.)

567t. Leonard Carlitz: Congruences connected with the power series expansions of the Jacobi elliptic functions.

Extending the results of a previous paper (Duke Math. J. vol. 16 (1949) pp. 297$302)$, it is shown first that $A^{m}(u)\left(A^{p-1}(u)-1\right)^{r} \equiv 0\left(\bmod \left(p^{m}, p^{r}\right)\right)$, where $u$ is an indeterminate, $p$ is a prime $>2$, and $\operatorname{sn} x=\operatorname{sn}(x, u)=\sum_{m=1}^{\infty} A_{m}(u) x^{m} / m !$. Similar results are also obtained for powers of $\operatorname{sn} x$. Next if $A_{m}(u)=\sum_{m=1} a_{m i} u^{i}$, then it is proved that $a_{m+t, i} \equiv(-1)^{t / 2} a_{m i}\left(\bmod p^{m}, p^{a-j}\right)$, where $p^{0-1}(p-1) \mid t, p^{j-1} \leqq i<p^{j}$. If $\beta_{m i}$ denotes the general coefficient in the expansion of $x / \mathrm{sn} x$, then $\beta_{m+t, i} /(m+t) \equiv(-1)^{t / 2}\left(\beta_{m i} / m\right)$ $\left(\bmod \left(p^{m-1}, p^{\sigma-i}\right)\right)$, provided $p-1 \nmid m$; a similar result is also obtained when $p-1 \mid m$. (Received June 16, 1952.)

\section{8t. Leonard Carlitz: Generalized Dedekind sums.}

Put $c_{r}(h, k)=\sum_{\mu_{m=1}}^{k} \bar{B}_{p+1-r}(u / k) \bar{B}_{r}\left(h_{\mu} / k\right)$, where $\bar{B}_{r}(x)$ denotes the Bernoulli function, $p$ is a fixed odd integer $>1$, and $(h, k)=1$. T. M. Apostol has proved a transformation formula for the function $G_{p}(x)=\sum_{1}^{\infty} n^{-p} x^{n} /\left(1-x^{n}\right)$ (Duke Math. J. vol. 17 (1950) pp. 147-157). Using this result we prove a reciprocity formula involving $c_{r}(h, k)$ which includes Apostol's reciprocity theorem for $c_{p}(h, k)$. We also generalize some theorems on Dedekind sums proved by Rademacher and Whiteman (Amer. J. Math. vol. 63 (1941) pp. 377-407). (Received July 21, 1952.)

569t. Leonard Carlitz: Invariantive theory of equations in a finite field.

Consider the reversible transformation $T: \eta_{i}=f_{i}\left(\zeta_{1}, \cdots, \zeta_{r}\right)(i=1, \cdots, r)$, where $f_{i}\left(x_{1}, \cdots, x_{r}\right) \in G F\left[q, x_{1}, \cdots, x_{r}\right]$, and $\zeta_{i}, \eta_{i} \in G F(q)$. Two polynomials $g, h \in G F\left[q, x_{1}, \cdots, x_{r}\right]$ are equivalent provided $T g=h$ for some $T$; thus the totality of polynomials is separated into a number of classes. The determination of this number is a simple combinatorial problem. Applications are made to the equation $f\left(\zeta_{1}, \cdots, \zeta_{r}\right)=\alpha$. (Received July 21,1952 .)

570t. Leonard Carlitz: Some sums connected with quadratic residues.

The well known result $\sum_{1}^{(p-1) / 2}(r / p)>0$ is generalized in the form $(-1)^{k} \sum_{r=1}^{(p-1) / 2}(r / p) E_{2 k}(2 r / p)>0(p \equiv 3(\bmod 4)),(-1)^{k} \sum_{r-1}^{(p-1) / 2}(r / p) E_{2 k}(2 r / p)>0$ $(p \equiv 1(\bmod 4))$, where $E_{m}(x)$ denotes the Euler polynomial. Similar results involving the Bernoulli polynomials also hold. (Received June 16, 1952.)

\section{Harvey Cohn: Stable lattices. II.}

Continuing in the terminology of the previous abstract (Bull. Amer. Math. Soc. Abstract 57-3-203), the author considers lattices representing all integers in a totally real field $K$, for which the norm function, $\phi=\left|x_{1} x_{2} \cdots x_{n}\right|$, has a stable minimum (under infinitesimal variations of the coefficients $a_{i j}$ ). Here $x_{i}=\sum a_{i j} m_{j}$ and $a_{i j}$ is the $i$-conjugate of the $j$-basis element. The condition for stability is that for any two conjugates, $i$ and $I$, a unit $u$ exist for which $u_{i} / u_{I}$ is negative. When $K$ is quadratic this just means there exists a unit of negative norm. When $K=R(\cos 2 \pi / N)$, the condition is satisfied for an extensive class of $N$ including (at least) prime powers. (Research sponsored by Army Ardnance.) (Received July 17, 1952.) 


\section{H. S. M. Coxeter: Hyperbolic honeycombs and indefinite quad-} ratic forms. Preliminary report.

Many indefinite forms $f(x, y, z, \cdots)$ are found to yield symmetrical patterns when the variables are interpreted as homogeneous coordinates in a hyperbolic space whose absolute quadric has the equation $f=0$. The symmetry operations of the pattern represent automorphs of the form. For instance, when $f=x^{2}+y^{2}-z^{2}-x z-y z$ [Markoff, Math. Ann. vol. 56 (1903) pp. 233-251], the solutions of the Diophantine equation $f=-1$ are the vertices of a tessellation of squares and hexagons filling the hyperbolic plane. Again, when $f=x^{2}+y^{2}+z^{2}-t^{2}-x t-y t-z t$ [Dickson, Studies in the theory of numbers, p. 143], the integral solutions of $f=-1$ are the vertices of a honeycomb of cubes and truncated octahedra filling hyperbolic 3-space. (Received June 19, 1952.)

\section{D. W. Dubois: Structure of Archimedean partly ordered fields.}

A field $F$ (commutative) is called a partly ordered field if $F$ has a nonempty subset $P$ of positive elements which is closed under addition, multiplication, and division. " $x>y$ " and " $y<x$ " are abbreviations for " $x-y \in P$." The partial order is Archimedean if for every $y \in F$, and $x \in P$, there exists an integer $n$ with $n x>y$. The following structure theorem is proved: If $F$ is Archimedian partly ordered, then there is a familyS of simple Archimedean orderings of $F$ such that the positive elements of $F$ are those which are positive according to every ordering belonging to $S$. This structure is revealed by showing that a certain Banach algebra $A$ is a subdirect sum of fields isomorphic with the field $R$ of all real numbers, where $A$ is the completion of $F$ relative to the norm $\mu(x)=\inf \{\gamma>0 ;-\gamma<x<\gamma\}, \gamma$ being a rational (every partly ordered field contains the ordered field of rationals). (Received July 11, 1952.)

\section{4t. Oscar Goldman: Ordered abelian groups. Preliminary report.}

If $G$ is a totally ordered abelian group, denote by $G_{+}$its set of non-negative elements. Let $L$ be the set of all complex-valued functions $f$ defined on $G_{+}$for which $\|f\|=\sum_{t} \in a_{+}|f(t)|<\infty$. Then $L$ forms a commutative Banach algebra under $\|f\|$ as norm, and convolution as multiplication; we call $L$ the group algebra of the ordered group $G$. The algebraic structure of $L$ reflects some of the order properties of $G$, as for example: $L$ has no zero divisors if, and only if, the ordering of $G$ is Archimedean. The space of maximal ideals $M$ of $L$, with the usual topology, has some interest. $M$ is compact, and, if one assumes the ascending chain condition on isolated subgroups of $G, M$ is also connected. (The converse of the latter statement has not yet been settled.) The elements of $M$ are in one-to-one correspondence with the order-characters of $G$, i.e., the complex-valued multiplicative functions on $G_{+}$of modulus not exceeding one, and assuming the value 1 at the zero element of $G$. Because of this, $M$ has a sort of analytic structure, and $L$ is an algebra of analytic functions. (Received July 21, 1952.)

\section{R. A. Good and D. R. Hughes: Associated groups for a semi- group.}

Let $S$ be a semigroup, $B$ a subsemigroup of $S$ satisfying $B S B C B$; then $B$ is called a bi-ideal of $S$. An ordered sequence (finite or denumerably infinite) of subsemigroups of $S$, each of which is a bi-ideal of the preceding semigroup, is called a bi-ideal chain of $S$. If and only if $S$ possesses a Kerngruppe in the sense of Suschkewitsch it possesses a finite bi-ideal chain with a member $B_{n}$ such that $B_{n}$ possesses no proper bi-ideals 
and is thus a group. $B_{n}$ is a bi-ideal of $S$ and is called an associated group for $S$. The index of an associated group is defined; all associated groups for $S$ have the same index and are isomorphic to one another. Every group homomorph of $S$ is a homomorph of the (abstract) associated group for $S$; under certain conditions the associated group for $S$ is a homomorph of $S$, and is thus a maximal group homomorph of $S$. (Received July 21, 1952.)

\section{Franklin Haimo: Some fixpoint properties of normal auto- morphisms. Preliminary report.}

Let $G$ be a group with center $Z$. Let $B_{1}(G)$ be the set of all elements of $G$ which are the common fixpoints for all the central automorphisms of $G$. If $B_{k}(G)$ is defined, construct $B_{k+1}(G)$ by $B_{k+1}(G) / B_{k}(G)=B_{1}\left(G / B_{k}(G)\right)$. A typical result is the following: For a central automorphism $\alpha$ of $G$, let $T_{\alpha}$ be the set of all $x^{-1} \alpha(x)$, where $x$ runs through $G$. Then $T_{\alpha} \subset Z$. Suppose that $\alpha$ restricted to $T_{\alpha}$ is the automorphism $x \rightarrow x^{-1}$, and suppose that there exists an integer $n \geqq 2$ such that $G=B_{n}(G)$. Then $\alpha^{2}$ is the identity automorphism on $G$. (Received July 21, 1952.)

577t. Melvin Henriksen and J. R. Isbell: On the continuity of the real roots of an algebraic equation.

While the root of an algebraic equation is a continuous multiple-valued function of its coefficients, there is no general theory of decomposition into single-valued functions. This paper shows that when a polynomial $P(x, w)=w^{n}+\sum_{k=0}^{n-1} a_{k}(x) w^{k}$ has coefficients which are continuous functions on a Hausdorff space $X$, and for each $x \in X$ there is at least one real root of $P(x, w)=0$, there is an open set $U$ on which a real root can be given by a continuous function. In case the number of real roots is constant over $X$, one of them can be given by a function continuous on $X$. With these results one can prove for normal spaces the following theorem, stated by Hewitt [Rings of real-valued continuous functions. I, Trans. Amer. Math. Soc. vol. 64 (1948) p. 76]: Let $C(X, R)$ be the ring of all continuous real-valued functions on a completely regular space $X$; let $M$ be a maximal ideal in $C(X, R)$. Then $C(X, R) / M$ is a real closed field. Hewitt's proof relies on the erroneous assumption that the least real root of a real polynomial is a continuous function of its coefficients [see Mathematical Reviews vol. 10 (1949) p. 126]. Since the present proof relies on the Tietze extension theorem, the question for completely regular spaces is not settled. (Received June 30, 1952.)

\section{I. N. Herstein: A generalization of a theorem of Jacobson. III.}

In the paper $A$ generalization of a theorem of Jacobson [Amer. J. Math. (1951) pp. 756-763] it was proved that if $R$ is a ring with center $Z$ such that $x^{n}-x \in Z$ for all $x \in R$ where $n$ is a fixed integer larger than 1 , then $R$ is commutative. In the second paper in this series this result was extended to the case where $n=n(x)$ might vary but where $n(x) \leqq M$ for all $x \in R$. In this paper the following unrestricted theorem is proved: Let $R$ be such that $x^{n(x)}-x \in Z$ for all $x \in R, n(x)$ depending on $x$. Then $R$ is commutative. The result is first established for the division ring case using a recent field theoretic theorem due to Marc Krasner (forthcoming). By the Jacobson structure theorems the semi-simple case is disposed of. The situation then hinges on the subdirectly irreducible case. The key result here is that all commutators are in the center. From there, by a series of theorems, the problem is reduced to the situation where $n(x)$ is constant. The theorem is known to be true here, settling the general situation completely. (Received July 17, 1952.) 


\section{D. G. Higman: Focal series in finite groups.}

For $S$ a subgroup of the [finite] group $G$, we define a descending chain of normal subgroups i $S$ of $S$, which we term the focal series of $S$ in $G$, by the recursive formulas: $0 S=S, i+1 S=$ the subgroup generated by all commutators $c=[s, g]$ with $c$ and $s$ in $S, g$ in $G$. Let $p$ be a set of primes, and denote by $P(\mathfrak{p})$ the subgroup generated by all those elements in $G$ whose orders have no prime divisors in $\mathfrak{p}$. The central result of our investigation is: if for some $i, p$ contains all the prime divisors of the index $[S: i S]$, then every prime which divides $\left[P(\mathfrak{p}) \wedge S: P(\mathfrak{p}) \wedge \wedge_{i} S\right]$ also divides $[P(\mathfrak{p}): P(\mathfrak{p}) \wedge S]$. Hence, for instance, if $[G: S]$ and $\left[S:{ }_{i} S\right]$ are relatively prime, there exists a normal subgroup $N$ of $G$ such that $G=N S$ and $i S=N \wedge S$. (Received July 16,1952.)

\section{0t. J. R. Jackson: $A$ partial ordering defined by certain matrices.}

Let $\left(p_{m n}\right)$ be an $M \times N$ matrix with real entries. Write $m<{ }^{*} m^{\prime}$ if, for some $n$ $p_{m n}<0<p_{m^{\prime} n}$; and let " $<$ " be the extension of " $<*$ " to be transitive. It is shown that the relation " $\prec$ " is a partial ordering of the integers $1,2, \cdots, M$, if and only" if the following condition is satisfied: $\left(p_{m n}\right)$ can be replaced, by means of interchanges of rows and interchanges of columns, by a matrix $\left(q_{m n}\right)$ with the property that if $q_{\mu \nu}>0$, then whenever $m \leqq \mu$ and $n \leqq \nu$, we have $q_{m n} \geqq 0$. The result is of interest from the point of view of a mathematical description of industrial production scheduling. (Received July 17, 1952.)

\section{1t. R. E. Johnson: Representations of prime rings.}

This paper continues the study of prime rings started in an earlier work (Prime rings, Duke Math. J. vol. 18 (1951) pp. 799-809). It is assumed that the prime ring $R$ has a set $\Re$ of $r$-ideals and a set $R$ of $l$-ideals satisfying the seven closure assumptions of the earlier paper. If we select atoms $M$ from $\mathfrak{R}$ and $N$ from $\Re$ such that $M \cdot N \neq 0$, then the ring $K=M \cap N$ is an integral domain, and $M$ is an $(R, K)$-module while $N$ is a $(K, R)$-module. It is shown that the lattice $\Re$ is isomorphic to a lattice of $K$-submodules of $M$, and dually for $\&$ and $N$. Finally, it is shown that for any set $\left\{x_{1}, \cdots, x_{n}\right\}$ of $K$-linearly independent elements of $N$, and any other set $\left\{y_{1}, \cdots, y_{n}\right\}$ of elements of $N$, there exist an $a$ in $R$ and a nonzero $k$ in $K$ such that $x a=k y_{i}, i=1, \cdots, n$. (Received July 22, 1952.)

582. Jan Kalicki and D. S. Scott: Equational completeness of $a b$ stract algebras. Preliminary report.

Terminology will be that of J6nsson and Tarski, Boolean algebras with operators, Part I (Amer. J. Math. vol. 73 (1951) in particular pp. 894-897). Let Eq (K) be the set of all equations $f=g$ between algebraic functions $f$ and $g$ over a similarity class $K$. Let Id $\left(\mathfrak{A}_{K}\right)$ be the set of equations $f=g$ in $\mathrm{Eq}(K)$ such that $f_{\mathfrak{P}_{K}}=g_{\mathfrak{U}_{K}}$ holds in the algebra $\mathfrak{A}_{K}$ of the similarity class $K$. $\mathfrak{A}_{K}$ is equationally complete $\left(\mathfrak{A}_{K} \in E C\right)$ if (1) Id $\left(\mathfrak{A}_{K}\right) \neq \mathrm{Eq}(K)$ and (2) for any algebra $\mathfrak{B}_{K}$ if $\operatorname{Id}\left(\mathfrak{A}_{K}\right) \subset \mathrm{Id}\left(\mathfrak{B}_{K}\right)$, then $\operatorname{Id}\left(\mathfrak{P}_{K}\right)$ $=\mathrm{Eq}(K)$. It has been shown that: (a) If Id $\left(\mathfrak{A}_{K}\right) \neq \mathrm{Eq}(\boldsymbol{K})$, then there is an algebra $\mathfrak{B}_{K} \in E C$ such that $\operatorname{Id}\left(\mathfrak{A}_{K}\right) \subseteq \operatorname{Id}\left(\mathfrak{B}_{K}\right)$. (b) An algebra $\mathfrak{A}=\langle A, f\rangle$ with one associative binary operation $f$ is equationally complete if and only if one of the following conditions holds for any $w, x, y, z \in A$ : (1) $f(x, y)=x$; (2) $f(x, y)=y$; (3) $f(x, y)=f(w, z)$; (4) $f(x, f(y, z))=f(f(x, y), z), f(x, y)=f(y, x)$, and $f(x, x)=x ;(5) f(x, f(y, z))=f(f(x, y), z)$, $f(x, y)=f(y, x), f\left(x^{p}, y\right)=y$ where $x^{1}=x, x^{i+1}=f\left(x^{i}, x\right)$, and $p$ is a prime. (c) As a simple corollary to (b) it is seen that every equationally complete algebra with one associative binary operation has a finite equational basis. (Received July 21, 1952.) 


\section{3t. Irving Kaplansky: Dual modules over a valuation ring.}

Several authors (Pruffer, Krull, Vilenkin, and others) have studied linearly compact modules over the $p$-adic integers, that is, topological modules with the finite intersection property for cosets of closed submodules. The author shows that these are dual to discrete modules; the duality must be taken, not relative to the reals mod one, but to its primary subgroup (Prüfer's group of type $p^{\infty}$ ). Thus the theory of linearly compact modules coincides with that of ordinary modules. This is the starting point for a more general study of such duality. (Received June 12,1952.)

\section{Leo Katz and Ingram Olkin: Properties and factorizations of matrices defined by the operation of pseudo-transposition.}

A matrix $C, n \times n$, is $(p, q)$ pseudo-orthogonal if it satisfies the relation $C J C^{\prime}=J$, where $J=I_{p}+\left(-I_{q}\right),+$ is the direct sum, and $p+q=n$. Under a pseudo-orthogonal transformation, the quadratic form $\sum_{1}^{p} x_{i}^{2}-\sum_{1}^{a} y_{i}^{2}$ is invariant. Lee (Quarterly Journal of Mathematics, 1944) and Hsu (Quarterly Journal of Mathematics, 1946) have obtained factorizations of such matrices. By defining the operation of pseudotransposition $\left(C^{0}=J C^{\prime} J\right.$ is the pseudo-transpose of $\left.C\right)$, unified definitions, analogous to the definitions using ordinary transposition, are obtained for pseudo-symmetric, pseudo-skew, and pseudo-orthogonal matrices. Analogues of certain theorems involving transposition are proved. These include a new factorization of a pseudo-orthogonal matrix in terms of a pseudo-skew matrix and the analogue of the Toeplitz factorization. The matrices considered are real. (Received July 21, 1952.)

\section{5t. Frank Kozin: On functions of three vectors.}

Let $f(x, y, z)$ be a real function of three vectors belonging to vector spaces of dimensions $>0$. If $f$ is subadditive in $x$ and $y$, and superadditive in $x$ and $z$, then $f$ is additive in $x$ and $y$ as well as in $x$ and $z$. Let $\left\|a_{i k}\right\|$ be a square matrix of order $n$. If $x=\left\{a_{11}\right\}, y=\left\{a_{12}, \cdots, a_{1 n}\right\}, z=\left\{a_{21}, \cdots, a_{2 n}\right\}$, and $f(x, y, z)$ denotes the determinant $\left|a_{i k}\right|$ where $a_{i k}(i, k=2, \cdots, n)$ are kept constant, then the preceding theorem yields the sufficiency of Menger's postulates for determinants (C.R. Acad Sci. Paris vol. 234 (1952) pp. 1941-1943). (Received July 18, 1952.)

\section{6t. W. J. LeVeque: Note on $S$-numbers.}

The transcendental number $\xi$ is said to be an $S$-number if there are constants $\Gamma$ and $\gamma$ such that the inequality $\left|x_{0}+x_{1} \xi+\cdots+x_{n} \xi^{n}\right|>\Gamma\left(\max \left(\left|x_{0}\right|, \cdots,\left|x_{n}\right|\right)\right)^{-\gamma n}$ holds for all $n$ and all sets of rational integers $\left(x_{0}, \cdots, x_{n}\right) \neq(0, \cdots, 0)$. Mahler showed that almost all real numbers (complex numbers) are $S$-numbers, and conjectured that this is even true with $\gamma=1(\gamma=1 / 2)$. It is shown here that almost all real numbers (complex numbers) are $S$-numbers for which it is possible to take $\gamma=2(\gamma=3 / 2)$; this improves a result due to Koksma, that it is possible to take $(\gamma=3)(\gamma=5 / 2)$. (Received June 20, 1952.) $k(i)$.

587t. W. J. LeVeque: Continued fractions and approximations in

The results of the above paper are applied to two problems. The trick is to find an algorithm for producing the best Gaussian rational approximations to an arbitrary complex number $\xi$; a regular continued fraction is constructed, all of whose convergents are best approximations to $\xi$, and among whose convergents are to be found all solutions of the inequality $|\beta(\beta \xi-\alpha)|<0.48 \cdots$ in Gaussian integers $\alpha$ and $\beta$. 
The second question, how well most complex numbers can be approximated by Gaussian rationals, is answered by the following theorem: the inequality $|\beta(\beta \xi-\alpha)|$ $\left\langle f(|\beta|)\right.$ has only finitely many solutions $\alpha, \beta$ for almost all $\xi$ if the integral $\int^{\infty} t f^{2}(t) d t$ converges, and has infinitely many solutions for almost all $\xi$ if this integral diverges. Here $f$ is a positive function satisfying suitable monotonicity conditions. Metric results are also obtained concerning the order of magnitude of the norms of the denominators of the continued fraction mentioned above. (Received June 20,1952.)

588t. W. J. LeVeque: Geometric properties of Farey sections in $k(i)$.

The work of Cassells, Ledermann, and Mahler on complex Farey sections is extended in several directions, the principal new tool being a simple geometric interpretation of the boundaries of the regions associated with Farey elements, in terms of spheres tangent to the complex plane at the Farey points. In particular, the theorem proved by Cassells et al., that these regions are star domains about the Farey points, is here proved much more simply. (Received June 20, 1952.)

\section{9t. W. J. LeVeque: On n-dimensional uniform distribution mod- ulo 1.}

Koksma has considered the distribution modulo 1 of sequences $\{f(n, \theta)\}$ depending on a real parameter $\theta$, and has shown that under suitable restrictions such a sequence is uniformly distributed modulo 1 for almost all $\theta$ in an appropriate interval. The present author gave a different proof of certain of Koksma's results, basing the proof on properties of quasi-orthogonal functions pointed out by Kac, Salem, and Zygmund. This method is here applied to $m$-fold sequences $\left\{f_{1}\left(n_{1}, \cdots, n_{r}, \theta_{1}, \cdots, \theta_{s}\right), \cdots\right.$, $\left.f_{m}\left(n_{1}, \cdots, n_{r}, \theta_{1}, \cdots, \theta_{s}\right)\right\}$ depending on $r$ integral variables and $s$ continuous variables. Analogous but more complicated theorems are proved. (Received June 20,1952.)

\section{0t. Jakob Levitzki: On the structure of $P$-soluble rings.}

A ring is called a $P$-ring if it has no nilpotent $(\neq 0)$ elements modulo its maximal nil ideal. The sum of all right $P$-ideals of a ring $S$ is a two-sided ideal called the $P$-socle of $S$. Iterating transfinitely the process of forming the $P$-socle one obtains an ascending chain of ideals $\left\{A_{\sigma}\right\}$ culminating, say, at $A_{\tau}$. If $S=A_{\tau}$, then $S$ is called $P$-soluble. In the present paper $P$-soluble $I$-rings (i.e. rings with nonzero idempotents in every non-nil right ideal) are studied, and e.g., a necessary and sufficient condition for the $P$-solubility of an $F I$-ring (i.e., a ring whose homomorphic images are $I$-rings) is found. This condition can be formulated as a weak minimum condition imposed on a certain class of principal right ideals. It turns out that an $F I$-ring is $P$-soluble if and only if every primitive image is $P$-soluble. Special cases are $F I$-rings with bounded index and more generally such with a bounded index modulo every primitive ideal, which were studied in the author's paper On the structure of algebraic algebras and related rings (to appear in Trans. Amer. Math. Soc.). These results are applied to the problem of the local finiteness of algebraic algebras and yield a generalization of Kaplansky's results on the problem of Kurosch. (Received August 12, 1952.)

591t. J. E. McLaughlin: The normal completion of complemented modular lattices. Preliminary report.

Let $L$ be a complemented modular point lattice, $L_{i}$ its ideal completion, $L_{\nu}$ its normal completion. Then it is shown that $L_{\nu}$ is modular if and only if $L_{\nu}$ is isomorphic to a sublattice of $L_{i}$. It is also shown that if $L$ is orthocomplemented, then 
$L_{\nu}$ is orthocomplemented. This gives as a corollary the following partial extension of the Stone-Glivenko Theorem for Boolean algebras: the normal completion of an orthocomplemented modular point lattice with the finite dependence property on points is an orthocomplemented modular point lattice. An example is given of an orthocomplemented modular point lattice whose normal completion is not modular. (Received July 21, 1952.)

\section{E. V. Schenkman: A generalization of the central elements of a group.}

For elements $a$ and $g$ in a group $G$, set $g^{-1} a g=a(g)$ or $a^{(1)}(g)$ and set $a^{(n)}(g)$ $=a^{(n-1)}(g)$. If, for some $n$ and every $g \in G, a^{(n)}(g)=a, a$ is said to be weakly selfconjugate. The following results then hold: (1) if in a locally finite group $G$ every element of prime power order $p$ is weakly self-conjugate, then the set of such elements comprise a normal subgroup of $G$. (2) An element is in the nil radical of a locally finite solvable group if and only if it is weakly self-conjugate. (Received July 21, 1952.)

\section{H. E. Stelson: Finding the root of an equation by iteration.}

Assume that a real root of $x=f(x)$ has been isolated and that the sequence $x_{1}, x_{2}, x_{3}, \cdots$ has been computed from an initial value, $x_{0}$, near the root. For $f(x)$ a decreasing function (an increasing function can be transformed into a decreasing function), we have a root between $x_{n}$ and $x_{n+1}$. An upper and lower limit for the root may be obtained by use of the formula $x=x_{i}-\left(\Delta x_{i}\right)^{2} / \Delta^{2} x$ for the points $\left(x_{0}, f\left(x_{0}\right)\right)$, $\left(x_{1}, f\left(x_{1}\right)\right)$ and for the points $\left(x_{0}, f\left(x_{0}\right)\right),\left(x_{2}, f\left(x_{2}\right)\right)$. These limits may be improved by use of the formula $x=x_{i}-\left(\Delta x_{i}+\Delta x_{i+1}\right) /\left(\Delta x_{i+1} / \Delta x_{i}-\left(\Delta x_{i} / \Delta x_{i+1}\right)^{2} \Delta^{2} x_{i+1} / \Delta^{2} x_{i}\right)$ for the points $\left(x_{0}, f\left(x_{0}\right)\right),\left(x_{1}, f\left(x_{1}\right)\right),\left(x_{2}, f\left(x_{2}\right)\right)$ and $\left(x_{1}, f\left(x_{1}\right)\right),\left(x_{2}, f\left(x_{2}\right)\right),\left(x_{3}, f\left(x_{3}\right)\right)$ which is derived by successive approximations. $f^{n}(x)$ must be of constant sign throughout the interval considered. Accuracy can be estimated by means of $R_{n+1}$ for Newton's ordinary interpolation formula for divided differences. (Received March 20, 1952.)

\section{4t. G. L. Walker: Fermat's theorem for algebras.}

If $A$ is an algebra over $F$ and $x$ is an indeterminate, let $I(A)$ be the set of all $f(x)$ in $F[x]$ such that $f(a)=0$ for all $a$ in $A . I(A)$ is then the principal ideal in $F[x]$ consisting of zero and all polynomial identities for $A$ in a single indeterminate. We prove: if $A$ is a total matrix algebra of order $m^{2}$ over $G F\left(p^{n}\right)$, then $I(A)$ is the principal ideal generated by $f=f_{n} f_{2 n} \cdots f_{m n}$ where $f_{i}=x^{p^{i}}-x$. When $m=1$, this is the Fermat theorem. This result is extended to semi-simple algebras of characteristic $p$ and finally to the theorem: if $A$ is an algebra over $F$ with radical $N$, if $I(A-N)=(f), f$ in $F[x]$, and $I(N)=\left(x^{r}\right)$, then $\left(g_{1}\right) \leqq I(A) \leqq\left(g_{2}\right)$ where $g_{2}=[f(x)] r$ and $g_{2}=1 \mathrm{~cm} x^{2}, f(x)$. An example shows that these bounds on $I(A)$ cannot be improved without additional hypothesis. (Received July 18, 1952.)

\section{5t. A. D. Wallace: Topological semigroups (Local stability. I).}

A TSG is a map (=continuous function) $\mu: S \times S \rightarrow S$ such that $S$ is Hausdorff and $\mu$ is associative. As usual $x y=\mu(x, y)$. If $a \in S$ and $T C S$, let $T^{a}$ be the intersection of all sets $U \cdot T, U$ an open set including $a$. If $S$ is locally compact and $T$ is compact then $T^{a}=a \cdot T$. (If $S$ is a TG and $e$ is the unit, then $T^{e}=\bar{T}$ ). Also $\square^{a}=\square, T_{1}^{a} \subset T_{2}^{a}$ if $T_{1} \subset T_{2} \cdot\left(T^{b}\right)^{a} \subset T^{a b},\left(T_{1} \cup T_{2}\right)^{a}=T_{1}^{a} \cup T_{2}^{a}$. (If $e$ is a left unit for $S$ then $T C T^{a}$ so $e$ is a closure operator for $S$.) If $S$ is a regular space, then $\left(T^{a}\right)^{-} C\left(T^{-}\right)^{a}$ so $T^{a}$ is closed if $T$ 
is closed. Note that $a T \subset T^{a}$. We say that $T$ is locally left stable at $a$ if $T=T^{a}$. If $S$ is compact and is locally left and right stable at a set dense in $S$, then $S$ is a topological group. We note that these concepts and results are partially applicable to topological dynamics, i.e., when $\mu: S \times X \rightarrow X$ is such a map that $\mu\left(s_{1}, \mu\left(s_{2}, x\right)\right)$ $=\mu\left(s_{1} s_{2}, x\right)$. (Received July 7, 1952.)

\section{6t. L. R. Wilcox: Addition and harmonic addition in a field. Pre- liminary report.}

A natural beginning of the study of the algebra of electric networks under the binary operations of series and parallel connections is the determination of interrelations between the operations of addition and harmonic addition $a * b \equiv a b(a+b)^{-1}$ in a commutative field $F^{\prime}$. First, an ideal element $\infty$ is appended to $F^{\prime}$ satisfying various conditions such as $a \cdot \infty=\infty(a \neq 0), a+\infty=\infty$, etc. If $a, b \in F \equiv F^{\prime}+\{\infty\}$, then $a+b, a b, a * b,-a$ are defined so that (a) $F-\{0\}$ is a group under $*$ with $\infty$ as identity and $-a$ serving as inverse of $a$, and (b) duality between,$+ *$ is complete. Central in the theory is a relation $\sim$ between pairs of elements of $F$, where $(a, b)$ $\sim(c, d)$ means either $(a+d) *(c+b)=a * c+d * b$ or $b=a=-c=-d$. Many properties of $\sim$ are found, including those expressing reflexivity, symmetries, transitivity, and self-duality. Conversely, the properties found characterize fields in the following sense. Given a system $(F,+, *)$ having these properties, define multiplications $\times_{k}$ by $a \times_{k} b \equiv 2^{-1}\{a *(k-a)+b *(k-b)-(a+b) *(k-(a+b))\}$, where $2^{-1} x$ means $x * x$. (Here the system must not have characteristic 2 in an obvious sense.) For fixed $k \neq 0, \infty,\left(F-\{\infty\},+, X_{k}\right)$ is a field in which $k$ is the multiplicative unit and in which $k \times_{a} k$ serves as reciprocal of $a \neq 0$. In each such field, harmonic addition coincides with $*$. (Received July 21, 1952.)

\section{ANALYsis}

\section{H. A. Antosiewicz: Forced periodic solutions of systems of dif- ferential equations.}

The vector differential equation $d x / d t=A(t) x+p(x, t)+q(x, t, k)$ is considered under the following assumptions: (i) $x$ is a vector in $n$-dimensional space $E_{x}$; (ii) $A(t)$ is a continuous and periodic matrix of period 1 ; (iii) $p(x, t)$ is continuous in $(x, t)$ and periodic in $t$ of period 1 for $x$ in some fixed closed sphere $\bar{S}(0, R)$ and every $t$, satisfies a Lipschitz condition in $\bar{S}$, and $p(0, t) \equiv 0$; (iv) $q(x, t, k)$ is continuous in $(x, t, k)$ and periodic in $t$ of period 1 for $x$ in $\bar{S}$ and every $t$ and every real $k$, satisfies a Lipschitz condition in $\bar{S}$, and $q(x, t, 0) \equiv 0$. It is shown that if the characteristic exponents of $d x / d t=A(t) x$ are all less than unity, then there exists a positive definite quadratic form $V(x)$ such that for sufficiently small $k$ a region $V$ of $E_{x}$ bounded by $V(x)=C$ is mapped into itself by $T: x(1) \rightarrow x(0)$. It follows from Brouwer's fixed point theorem that there exists in $V$ at least one periodic solution of period 1 . This periodic solution is shown to be asymptotically stable in Liapounoff's sense. (Received July 21, 1952.)

\section{Nachman Aronszajn and A. N. Milgram: Differential oper- ators on Riemannian manifolds.}

The consideration of differential problems with general boundary conditions in a Euclidean domain requires the study of intrinsic differential operators on the boundary. The authors attack this question by considering differential operators on a general Riemannian manifold $\mathfrak{m}_{n}$. The notions of differential operators and $o$-forms 
(i.e. exterior differential forms with bilinear differential operators for coefficients) and their restriction to submanifolds are defined. The following results, among others, are obtained: (1) a closed $o$-form is an exact differential of an $o$-form; (2) the representation of an operator and its adjoint in terms of covariant derivatives; (3) analysis of boundary operators on an $(n-1)$-dimensional submanifold $\mathfrak{m}_{n-1}$ in connection with a differential operator defined in a neighborhood of $\mathfrak{m}_{n-1}$, in particular a complete description of all self-adjoint or positive systems among all normal systems of boundary operators. (Received July $14,1952$. )

\section{9t. I. E. Block: On Hilbert transforms.}

Let $\Lambda^{*}(-\infty,+\infty)$ be the class of continuous functions $f(x)$ such that $\mid f(x+h)$ $+f(x-h)-2 f(x) \mid \leqq A h$ uniformly in $x$ as $h \rightarrow 0$. Such a class was defined by Zygmund (Duke Math. J. vol. 12 (1945) pp. 47-76) to replace the class Lip 1 of functions. The following theorem is proved: Let $f(x)$ belong to $L^{p}(-\infty,+\infty), p>1$, and $\Lambda^{*}(-\infty,+\infty)$. Then the Hilbert transform of $f(x)$ belongs to $L^{p}(-\infty,+\infty)$ and $\Lambda^{*}(-\infty,+\infty)$. The method of proof follows the proof of Zygmund for a similar theorem for functions in $\Lambda^{*}$ on the unit circle. (Received June $\left.9,1952.\right)$

600. R. P. Boas: Functions of bounded characteristic and functions of exponential type.

The author coordinates a number of recent results concerning functions of bounded characteristic and functions of exponential type. Considerable simplification is achieved in the discussion of functions of exponential type with zeros $z_{n}$ such that $\sum\left|I\left(1 / z_{n}\right)\right|$ converges. Systematic use is made of a theorem of Ahlfors and Heins [Ann. of Math. (2) vol. 50 (1949) pp. 341-346] on the existence of $\lim r^{-1} \log \left|f\left(r e^{i \theta}\right)\right|$ for a function of exponential type which is bounded on the real axis. This theorem is extended to functions satisfying less restrictive conditions on the real axis; thus results of B. Levin [Doklady Akad. Nauk SSSR. N.S. vol. 71 (1950) pp. 601-604] are made sharper. The author does not consider Levin's proofs completely convincing. (Received July 16, 1952.)

\section{1t. Joseph Bram: Normality of rings of operators of type I.}

A ring $A$ of operators on a Hilbert space $H$ is called normal if for every subring $B$ containing the center $Z$ of $A$, we have $\left(B^{\prime} \cap A\right)^{\prime} \cap A=B$, where $E^{\prime}$ denotes the set of all operators on $H$ which commute with every operator in $E$. von Neumann showed that every factor of type $I$ is normal. To show that every ring of type $I$ is also normal, it suffices to prove the result for the case in which $A$ is, for some cardinal $n$, a "full matrix ring of order $n$ " over a commutative ring, since every ring of type $\mathrm{I}$ is a direct sum of such rings. The space $H$ is replaced by a smaller space $H_{1}$ on which $A$ is represented faithfully and on which $A^{\prime}$ is exactly the center of $A$. It is known that every subring of $A$ is weakly closed even when acting on $H_{1}$. The desired result is seen to be true on $H_{1}$, hence also on $H$. (Received June 1, 1952.)

602. George Brauer: Sets of convergence of ordinary Dirichlet series. Preliminary report.

It is shown that for any real number $\alpha$, each set of type $F_{\sigma}$ on the line $\sigma=\alpha$ is the set of convergence of an ordinary Dirichlet series of the form $\sum_{n=1}^{\infty} a_{n} n^{-s}, s=\sigma+i \tau$. Each denumerable set on the line $\sigma=\alpha$ is the set of divergence of an ordinary Dirichlet series. (Received July 15, 1952.) 
603. F. E. Browder: Assumption of boundary values in the Dirichlet problem for the general linear elliptic differential equation.

Let $K$ be a suitably differentiable linear elliptic differential operator of order $2 m$ on the bounded domain $D$ of $E^{n}$. Suppose that $u$ is a solution of the Dirichlet problem for the equation $K u=h$ with boundary value function $g, P$ a point of the boundary of $D$. It is shown that if $g$ and $h$ are sufficiently differentiable in the neighborhood of $P$ and if the boundary of $D$ is sufficiently smooth at $P$, then $u$ assumes its full set of boundary data at $P$. For $n=2$, a sufficient smoothness condition is that the boundary of $D$ should be locally connected at $P$ while $P$ is not an isolated point of the boundary. (Received July 21, 1952.)

\section{4t. F. E. Browder: Higher order boundary value problems for linear elliptic differential equations.}

Let $K$ be a suitably differentiable, strongly elliptic, linear differential operator of order $2 m$ on the bounded domain $D$ of $E^{n}, K=L+A$ where $L$ is self-adjoint and $A$ of less order than $2 m$. Let $\gamma$ be a set of boundary conditions and $\Gamma$ the subset of $C^{2 m}(\bar{D})$ satisfying the boundary conditions $\gamma$. Suppose that on $\Gamma L$ is self-adjoint with respect to the inner product of $L^{2}(D)$ and $(u, v \in \Gamma)$, further that $(-1)^{m} \int_{D} u \cdot \bar{K} v d x=$ the sum of an $m$-inner product on $D$, a positive boundary integral, and a boundary integral of lower order. The semi-boundedness for $K$ on $\Gamma$ is established together with existence and differentiability theorems, for solutions of $K u=h$ with boundary conditions $\gamma$, which are analogous to the author's previous results on the Dirichlet problem. (Received July $21,1952$. )

605t. F. E. Browder: Linear parabolic differential equations of arbitrary order.

If $K$ is a suitably differentiable linear elliptic differential operator of order $2 m$ on the bounded domain $D$ of $E^{n}$, the solution $u(x, t)[x D, 0<t<\infty]$ of the equation $(-1)^{m+1} K u=\partial u / \partial t$ is said to be a solution of the initial value problem with data $g$ and $f$ if for each $t, u$ satisfies the Dirichlet boundary data of $g$ and as $t$ goes to zero $u(x, t)$ approaches $f(x)$ in $L^{2}(D)$. The existence of a solution to the initial value problem is established if $K$ is self-adjoint and it is shown that the solution is $2 m$ times differentiable in $x$ and infinitely differentiable in $t$. The Green's function is constructed and applied to other boundary value problems. The methods used are based upon the author's results on linear elliptic equations. (Received July 21,1952.)

\section{R. C. Buck: Essentially admissible sequences.}

Let $S$ be the space of complex sequences $\alpha=\left\{a_{n}\right\}$ for which $\|\alpha\|=\sup \left|a_{n}\right|^{1 /(n+1)}$ $<\infty$. Let $A$ be the set of those $\alpha$ which are essentially admissible in the sense that there exists an entire function $f(z)$ of exponential type obeying the growth restriction $f(i y)=O(\exp c|y|)$ for some $c<\pi$, and such that $f(n)=a_{n}$ for all large $n$. Necessary and sufficient conditions are obtained that a sequence $\alpha$ belong to the closure of $A$. It is also shown that $A$ has no interior points, and that there are arbitrarily large spheres in $S$, disjoint from $A$. (Received July 21, 1952.)

607. C. W. Burrill: Characterizing systems of continuous functions. Preliminary report.

Several writers have studied the problem of characterizing a topological space by 
means of the continuous functions on the space. The principal contributions of this paper are the following. (1) A completely regular (Hausdorff) space $X$ is characterized by the lattice of continuous real-valued functions together with the sublattice of bounded continuous real-valued functions in case the space satisfies the following condition: for every $b \in \beta(X)-X$ there exists a continuous real-valued function $g$ such that for every real number $\alpha, b € e[x \in X ; g(x) \leqq \alpha]$. Here $\beta(X)$ is the Cech $\beta$-compactification of $X$ and $e$ is the closure operation in $\beta(X)$. The proof closely follows that of I. Kaplansky in Bull. Amer. Math. Soc. vol. 53 (1947) pp. 617-623. (2) If $Z$ is a completely regular (Hausdorff) space satisfying condition $\mathrm{C}$, then certain ordered sets of equivalence classes of continuous real-valued functions will characterize $Z$. Here $\mathrm{C}$ is a condition of $\mathrm{E}$. W. Chittenden: if $a \in Z$ then there exists a continuous realvalued function $f$ such that $f(x)=0$ if and only if $x=a$. (Trans. Amer. Math. Soc. vol. 31 (1929) p. 317.) Property $C$ holds if $Z$ satisfies the first axiom of countability. (Received July $21,1952$.

608t. P. L. Butzer: Linear combinations of Bernstein polynomials.

Denote $\mathfrak{B}_{n}^{[0]}=\left[\mathfrak{B}_{n}^{f}(x)\right]^{[0]}=B_{n}^{f}(x)$ and $\left(2^{k}-1\right) \mathfrak{B}_{n}^{[2 k]}=2^{k \mathfrak{B}_{2 n}^{[2 k-2]}}-\mathfrak{B}_{n}^{[2 k-2]}, k=1,2,3, \cdots$, where $B_{n}(x)=B_{n}^{f}(x)=\sum_{\nu=0}^{n} f(\nu \mid n) C_{n, \nu} x^{\nu}(1-x)^{n-\nu}$ is the Bernstein polynomial corresponding to the function $f(x)$ defined on $[0,1]$. The polynomial $\mathfrak{B}_{n}^{[2 k]}(x)$ can be rewritten in the form $\alpha_{k} B_{2} k_{n}(x)+\alpha_{k-1} B_{2 k-1}(x)+\cdots+\alpha_{0} B_{n}(x)$ with constants $\alpha_{i}=\alpha_{i}(k)$ satisfying $\sum_{i=0}^{k} \alpha_{i}=1$. The linearcombination $\mathfrak{B}_{n}^{[2 k]}(x)$ is shown under certain conditions to approach $f(x)$ more closely than the Bernstein polynomials. For example, if $f(x)$ is defined on $[0,1]$ with $|f(x)| \leqq M$, then at points where the $2 k$ th derivative $f^{(2 k)}(x)$ exists, it is shown that $\left|\mathfrak{B}_{2 n}^{[2 k-2]}(x)-f(x)\right|=O\left(n^{-k}\right)$ and $\left|\mathfrak{B}_{n}^{[2 k]}(x)-f(x)\right|=o\left(n^{-k}\right), n \rightarrow \infty$, $k=1,2,3, \cdots$. Correspondingly, for the Bernstein polynomials, at points where $f^{\prime \prime}(x)$ exists, the order of approximation to $f(x)$ is only $O\left(n^{-1}\right)$, and the existence of derivatives of higher order of $f(x)$ cannot improve this order (Voronowskaja, S. Bernstein, C. R. (Doklady) Acad. Sci. URSS. (1932) pp. 79-92). Now if $f^{(2 k)}(x)$ is continuous with modulus of continuity $\omega_{2 k}(\delta)$ on $[0,1]$, it is shown that $\left|\mathfrak{B}_{n}^{[2 k]}(x)-f(x)\right|$ $\leqq \max \cdot\left\{C_{n}^{-k} \omega_{2 k}\left(n^{-1 / 2}\right), C^{\prime} n^{-k-1}\right\}$ where $C=C(k), C^{\prime}=C^{\prime}(k ; f)$. If $f(x)$ satisfies only a Lipschitz condition of order $\alpha, 0<\alpha \leqq 1$, then, in general, $\mathfrak{B}_{n}^{[2 h]}$ does not approximate $f(x)$ more closely than $B_{2} k_{n}(x)$ for which $\left|f(x)-B_{2} k_{n}(x)\right|=O\left(n^{-\alpha / 2}\right)$, (Popoviciu, Mathematica Cluj vol. 10 (1935) pp. 49-54). (Received June 25, 1952.)

609. Joshua Chover: On locally-homogeneous measures and Hilbert space decompositions. Preliminary report.

A condition of "local-homogeneity," which is a weakening of the condition of translation invariance, and an algebraic concept of support are introduced for measures on a group, and examples are discussed. Secondly, an abstract equivalence relation, which is coarser than the usual one, is defined for nonzero measures, and in particular for those with supports. The local-homogeneity condition serves to define a special instance of this relation which is useful in application to operator theory. By means of this relation, and using the analogy between ideals of measures and vector subspaces, it is shown that any self-adjoint operator on Hilbert space whose weighted spectrum is "absolutely continuous" with respect to the domain of the relation induces a unique decomposition of the space into invariant subspaces which have certain measure theoretical properties. This decomposition permits the rigorous definition of a concept of generalized eigenvalue expansion such as is required for various operators in the Dirac model for quantum mechanics. (Received July 7, 1952.) 
610. R. V. Churchill and C. L. Dolph: Inverse transforms of products of Legendre integral transforms.

Let $f(n)(n=0,1,2, \cdots)$ denote the Legendre integral transform of a function $F(x)$ on the interval $(-1,1), f(n)=\int_{-1}^{1} F(x) P_{n}(x) d x$. If $g(n)$ is the transform of a function $G(x)$, then the product $f(n) g(n)$ is the transform of the function $H(x)$ where $H(\cos \theta)$ is represented by the integral $\pi^{-1} \int_{0}^{\pi / 2} \sin \alpha d \alpha \int_{0}^{2 \pi} F[\sin \alpha \cos (\beta+\theta / 2)]$ - $G[\sin \alpha \cos (\beta-\theta / 2)] d \beta$. The integral $H$ corresponds to the convolution integrals for Fourier and Laplace transforms. Sufficient conditions for the above inverse transform of $f(n) g(n)$ are that $F(x)$ and $G(x)$ be continuous $(|x| \leqq 1)$. The methods and results of J. P. Vinti [Proceedings of the American Mathematical Society vol. 2 (1951) pp. 19-23] played an essential role in deriving the above formula. This convolution property not only makes it possible to extend tables of Legendre transforms but it also leads to closed forms of solutions of boundary value problems. (Received July $21,1952$.

\section{E. G. H. Comfort: $A$ new inequality.}

The inequality $\left(x^{r}+y^{r}\right)(x+y)^{r}-(x+y)^{2 r}-\left(2^{r+1}-2^{2 r}\right) x^{r} y^{r} \geqq 0$ is proved for $x \geqq 0$, $y \geqq 0,0 \leqq r \leqq 1$. If $x y>0$, a rearrangement of the inequality shows that the difference $\left(x^{r}+y^{r}\right)-(x+y)^{r}$ is not less than a positive constant, which is independent of $x$ and $y$, multiplied by the $r$ th power of the harmonic mean of $x$ and $y$. (Received July 14, 1952.)

\section{2t. John DeCicco: The theory of residues of polygenic functions.}

For a polygenic function $F(z)$ of class $C^{(m)}$ in the region $0<\left|z-z_{0}\right|<\rho$, the residue function is $R\left(z_{0}, r\right)=(1 / 2 \pi i) \int_{a} f(z) d z$, where $C$ is a positively sensed circle with center at $z_{0}$ and positive radius $r<\rho$. If $f(z)$ is $q$ harmonic so that $\nabla^{2 a} f(z)=0$ in the region, then $R\left(z_{0}, r\right)$ is an even polynomial in $r$ of degree $2 q-2$ at most. The residue of order $q$ of a polygenic function $f(z)$ at a point $z_{0}$ is the limit (if it exists) of the derivative of order $q$ of $R\left(z_{0}, r\right)$ with respect to $r^{2}$ as $r$ approaches zero. If $f(z)$ is polygenic of class $C^{(m)}$ at a point $z_{0}$ and if $1<n+2 \leqq m$, then $f(z) /\left(z-z_{0}\right)^{n+1}$ possesses residues at $z_{0}$ of orders 0 to $[(m-n) / 2]$ inclusive. If $0 \leqq q \leqq[(m-n) / 2]$, the residue of order $q$ is $(1 /(n+q) !)\left(\nabla^{2 q} / 2^{2 q}\right)\left(\partial^{n} f / \partial z_{0}^{n}\right)$. The concepts can be extended to the residues of a polygenic function for a finite set of distinct points. If $F(z)=f(z) /\left(z-z_{1}\right) \cdots\left(z-z_{n}\right)$, where $f(z)$ is polygenic of class $C^{(2)}$ for the set $S$ of distinct points $z_{1}, z_{2}, \cdots, z_{n}$, the residue of order zero of $F(z)$ is the divided difference $\left[z_{1} z_{2} \cdots z_{n}\right]$ of $f(z)$, and the residue of order one of $F(z)$ is the divided difference $\left[z_{1} z_{2} \cdots z_{n}\right]$ of $\partial^{2} f / \partial z \partial \bar{z}$ minus the sum of the divided differences $\left[z_{1} \cdots z_{k-1} z_{k+1} \cdots z_{n}\right]$ of the function $(\partial f / \partial \bar{z}) /\left(z-z_{k}\right)^{2}$ for $k=1, \cdots, n$. These results are extended to confluent divided differences. (Received June $18,1952$.

\section{R. J. Duffin: Discrete potential theory.}

A study is made of analogy and counter-analogy between the Laplacian operator and the corresponding difference operator relative to the regular rectangular lattice in space. Courant has shown that the analogue of the potential of a point charge may be represented as a triple Fourier transform. This function is investigated here in detail and its complete asymptotic expansion at infinity is determined. To do this it is first of all necessary to develop a theory of the asymptotic expansions of multiple Fourier transforms. In the two-dimensional case, it is found possible to evaluate explicitly the potential of a point charge. As an analogue of Gauss's mean value theo- 
rem, it is shown that the value of a discrete harmonic function is to a certain approximation the mean of its values at the lattice points in a concentric sphere. An analogue of Harnack's inequality for positive harmonic functions is found. The following corollary corresponds to Liouville's theorem: A positive function which satisfies the Laplace difference equation at all lattice points is a constant. (Received July 18, 1952.)

614t. R. S. Finn: On the solutions of elliptic systems of first order equations.

Solutions of elliptic systems of the form (1) $\alpha \phi_{x}+\beta \phi_{y}=\psi_{y}, \gamma \phi_{x}+\delta \phi_{y}=-\psi_{x}$ are considered. At each point $(x+i y)$ an upper bound for the eccentricity $e$ of the mapping $(x+i y) \rightarrow(\phi+i \psi)$ is determined by the magnitudes of the coefficients $\alpha, \beta, \gamma, \delta$. It is shown that if $e<K \log \left(1+x^{2}+y^{2}\right)$, then a nonconstant entire solution omits at most one finite value. A similar theorem has been proved by $\mathrm{H}$. Grötzsch (Leipziger Berichte vol. 80 (1928)) using other methods, under the assumption of uniformly bounded eccentricity. More generally, the domain of values achieved by any solution in a neighborhood of an isolated point is exactly that of an analytic function, provided that $e<K \log \left(1 / x^{2}+y^{2}\right)$ near the point. Theorems of similar type hold for the complex gradient $\phi_{x}-i \phi_{y}$ of a solution of an elliptic equation (2) $A \phi_{x x}+2 B \phi_{x y}$ $+C \phi_{y y}=0$. If (2) is nonlinear, and if $e<K\left(1+\phi_{x}^{2}+\phi_{y}^{2}\right)^{i} \log \left(1+x^{2}+y^{2}\right)$ for some integer $n$, then an entire solution for which $\phi_{x}-i \phi_{y}$ omits a nondegenerate continuum is a linear function $\phi(x, y)=a x+b y+c$. Applications are made to solutions of elliptic variational problems $\delta \iint F(p, q) d x d y=0$. It is shown that if $F_{p p}+F_{q q}<K$, the condition $|\phi|<K\left(\log 1 / x^{2}+y^{2}\right)^{1 / 2}$ is sufficient for removability of an isolated singularity. Estimates are obtained for the rate of growth of entire solutions and various generalizations of Liouville's theorem for harmonic functions are given. The proofs depend on a conformally invariant formulation of the equations and on a technique due to M. Shiffman for estimating growth of double-integral quadratic functionals. (Received July 18, 1952.)

\section{W. H. Fleming and L. C. Young: A notion of boundary for generalized parametric surfaces.}

A linear functional $L$ on the space of continuous parametric integrands $f(x, J)$ defined by $L(f)=\iint f[x(u, v), J(u, v)] d u d v$, where $x(u, v)=\left[x^{1}(u, v), \cdots, x^{m}(u, v)\right]$ is an "elementary" (e.g., Lipschitzian) mapping of $[0 \leqq u \leqq 1 ; 0 \leqq v \leqq 1]$ into $m$-space and $J(u, v)=\left[\partial\left(x^{r}, x^{s}\right) / \partial(u, v)\right]$, is called an elementary (parametric) surface. Weak limits of sequences of elementary surfaces are called generalized (parametric) surfaces [L. C. Young, Bull. Soc. Math. France vol. 79 (1951) pp. 59-85]. The g-boundary $\lambda_{L}$ of a generalized surface $L$ is obtained by restricting the domain of $L$ to the space of exact integrands. If $\lambda_{L}=0, L$ is termed closed; $L$ is basic closed if $L=L_{1}+L_{2}$ with $L_{1}, L_{2}$ closed implies $L_{1}=k L$. Every generalized surface whose $g$-boundary $\lambda$ bounds some elementary surface is weak limit of positive linear combinations of elementary surfaces bounded by integral multiples of $\lambda$. Given $b>0$, a sufficient condition that an exact $\phi(x, J)$ exists such that $f_{0}(x, J)+\phi(x, J)$ is positive definite for $|x| \leqq b$ is that $L\left(f_{0}\right)>0$ for all basic closed $L$. For $m=3$, the latter inequality holds if $f_{0}$ is semidefinite, semiregular, and satisfies a certain condition involving its zeros. (Received July 14, 1952.)

616t. K. O. Friedrichs: On the differentiability of solutions of elliptic differential equations. 
Let the vector-valued function $u(x)$, defined in an open set $\mathfrak{D}$ of $m$-dimensional space $x=\left\{x_{1}, \cdots, x_{m}\right\}$, be a solution of the differential equation $L u(x)$ $=\sum_{\rho, \sigma=0}^{r} \tilde{D}^{\rho} a_{\rho \sigma}(x) D^{\sigma} u(x)=f(x)$ of order $2 r$, in which $D$ is the gradient and $\widetilde{D}$ the negative divergence, so that $D^{\sigma} u$ stands for all $m^{\sigma}$ derivatives of $u$ of order $\sigma$ while $\tilde{D}^{\rho}$ transforms systems of $m^{\rho}$ functions into one function. Furthermore let $a^{\rho \sigma}(x)$ be an $m^{\rho}$ by $m^{\sigma}$ matrix, and $f(x)$ a given function. Assume that $\int u^{r}(x) a^{r r}(x) u^{r}(x) d x \geqq a \int u^{r} u^{r} d x$ for every system $u^{r}(x)$ of $m^{r}$ functions which vanish outside a compact subset of $\mathfrak{D}$, so that $L$ is elliptic in the restricted sense. With reference to a Hilbert norm, we assume that $u$ admits the operator $D$ in the "strong sense" $r$ times and that $a^{\rho \sigma}(x) D^{\sigma} u(x)$ admits the operator $\widetilde{D}$ in the "weak sense" $\rho$ times. Theorem 1 . If $f(x)$ admits $D$ in the strong sense $s$ times, the solution $u(x)$ admits $D$ in the strong sense $r+s$ times provided $a^{\rho \sigma}$ is in $\mathfrak{\mho}_{s}$. Corollary. $u(x)$ is in $\mathfrak{夭}_{k}$ if $s+r>k+m / 2$. The main tools are integral inequalities and approximation with the aid of mollifiers. (Received June 16, 1952.)

\section{W. B. Fulks: The Neumann problem for the heat equation.}

The author considers the solutions of the following boundary value problem. Let $U(P)=U(x, t)$ be a solution of (1) $U_{x x}=U_{t}$ in $R:\{0<x<1 ; 0<t<T \leqq \infty\}$ which takes on prescribed initial values $U(x, 0)$ and whose normal derivative takes on prescribed boundary values $U_{x}(0, t)$ and $U_{x}(1, t)$. He is particularly concerned with generalizations of this problem. Let $Q=(y, s)$ be a point on the boundary $B$ of $R$, and let $F(P, Q)$ be the (explicitly known) Green's function associated with the problem. He then obtains necessary and sufficient conditions on $U(P)$ in order that a solution of (1) can be represented in the form (2) $\int_{B} F(P, Q) d_{\mu}(Q)$. He further shows that $U(x, 0+)$ $=\mu^{\prime}(x)$ and $U_{x}(0+, t)=\mu^{\prime}(t-0)$ wherever these derivatives exist. He then gives necessary and sufficient conditions on $U(P)$ for (3) $U(P)=\int_{B} F(P, Q) f(Q) d Q$ and from (2) and (3) he extracts conditions which uniquely determine the solution of this boundary value problem. (Received July 10, 1952.)

\section{I. S. Gál: On the principle of condensation of singularities.}

In a recent paper [Ann. Institut Fourier, vol. 3, p. 24] the author introduced the concept of "asymptotically subadditive" sequences of homogeneous and bounded operations mapping a normed vector space into another one. As was shown there the principle of uniform boundedness remains valid for these operations. It is now proved that the principle of condensation of singularities can also be extended in the same generality: Let $\left\{u_{m n}(x)\right\}, m, n=1,2, \cdots$, be a double sequence of homogeneous and bounded operations mapping a complete vector space $E$ into a normed vector space $E^{\prime}$. Suppose that every single sequence $\left\{u_{m n}(x)\right\}, n=1,2, \cdots(m$ fixed $)$, is asymptotically subadditive, that is to say $\left\|u_{m n}(x+y)\right\| \leqq\left\|u_{m n}(x)\right\|+o\left(\left|u_{m n}\right| \cdot\|y\|\right)$ uniformly in $x, y \in E$ and $\inf _{y}\left[\left\|u_{m n}(x+y)\right\|+\left\|u_{m n}(x)\right\|-\left\|u_{m n}(y)\right\|\right] \geqq o\left(\left|u_{m n}\right|\right)$ as $n \rightarrow \infty$ where the infimum is taken for every $y \in E$ with $\|y\| \leqq 1$. The generalized principle of condensation is as follows: If, for every $m=1,2, \cdots$, there is an $x_{m} \in E$ such that $\lim$ sup $\left\|u_{m n}\left(x_{m}\right)\right\|=+\infty$ as $n \rightarrow \infty$, then there is a common element $x \in E$ having the property that lim sup $\left\|u_{m n}(x)\right\|=+\infty$ as $n \rightarrow \infty$ for every $m=1,2, \cdots$. (Received July 23, 1952.)

619t. Seymour Ginsburg: $A$ cardinal number associated with $a$ family of sets.

For each ordinal number $a<\alpha$, let $U_{a}$ be a family of subsets of a set $R_{a}$, partially ordered by set inclusion. Let $m d\left(U_{a}\right)$ be the smallest cardinal number which is the 
power of the union of a maximal family of incomparable elements of $U_{a}$. Let $c m d\left(U_{a}\right)$ be the smallest cardinal number which is the $m d(V)$ of some coinitial subfamily $V$ of $U_{a}$. The following two results are shown: (1) $\prod_{a<\alpha} c m d\left(U_{a}\right)=c m d\left(\prod_{a<\alpha} U_{a}\right)$, where by $\Pi_{a<\alpha} U_{a}$ is meant the cartesian product. (2) If $U_{a}$ is a ramified family of sets, then $m d\left(U_{a}\right)=c m d\left(U_{a}\right)$. (Received July 15,1952 .)

\section{0t. Seymour Ginsburg: A class of everywhere branching sets.}

Let $P$ be an everywhere branching partially ordered set (see Day, Oriented systems, Duke Math. J. vol. 11 (1944) p. 201 ff.). Let $A(x)=\{y \mid y \leqq x, y \in P\} . P$ is said to have sufficiently many non-coinitial subsets (smns) if, for each pair of elements $p$ and $q$, in $P$ at least one of the following two conditions is false: (1) $A(p)$ is coinitial in $A(q)$, and (2) $A(q)$ is coinitial in $A(p)$. If $P$ is the dual of a ramified system, then $P$ is shown to contain a coinitial subset $S$ which has smns. A residual subset $Q$ of $P$ is called maximal residual if $Q$ is no proper coinitial subset of any residual subset of $P$. Let $F(P)$ be the family of maximal residual subsets of $P$, partially ordered by set inclusion. $F(P)$ has smns. If $P$ has smns, then $P$ is coinitially similar to $F(P)$. If two everywhere branching partially ordered sets, $P_{1}$ and $P_{2}$, are coinitially similar, then $F\left(P_{1}\right)$ is isomorphic to $F\left(P_{2}\right)$. (Received July 15,1952 .)

\section{1t. Seymour Ginsburg: $A$ theorem on similarity transformations.}

Each set considered is simply ordered, and each function a similarity transformation. The product of two sets is to be ordered by first differences. Theorem: Let $g$ map $C \times D$ into $E \times F$ and have the property that for each element $y$ in $C$, there are at least two elements in $E, u(y)$ and $v(y)$, where $u(y)<v(y)$, such that the set intersection of $g(y \times D)$ and $(u(y) \times F)$ is nonempty, and the set intersection of $g(y \times D)$ and $(v(y) \times F)$ is nonempty. Then (a) for a given element $p$ in $C$ and $q$ in $E$, where $u(p) \leqq q \leqq v(p)$, a function $h$, of $C$ into $E$, can be found so that $h(p) \neq q$, and for each element $y$ in $C$, either $h(y)=u(y)$ or $h(y)=v(y)$; and (b) if $C=E$, then a function $h$ of $E$ into $E$ can be found so that for each element $y$ in $E, h(y) \neq y$ and $u(y) \leqq h(y) \leqq v(y)$. Applications are the following: (1) Let $E$ have the property that for each function $f$ of $E$ into $E$, a point $p$ can be found so that $f(p)=p$. Then $(E, E)$ is an $A$-pair (see Arens: Ordered sequence spaces; Portugalia Math. vol. 10 (1951) pp. 25-28). (2) Let $p$ be a fixed point of $E$, and $F$ a set in which each point is fixed. Then for each element $y$ in $F,(p, y)$ is fixed in $E \times F$. (Received July 15,1952 .)

\section{2t. Seymour Ginsburg: Sets of incomparable elements in coinitial} subsets of a partially ordered set.

For each ordinal number $a<\alpha$, let $P^{a}$ be a partially ordered set with no minimal element. By $\operatorname{cs}\left(P^{a}\right)$ is meant the smallest cardinal number $m$ which has the following property: For some coinitial subset $Q^{a}$ of $P^{a}, m$ is the smallest cardinal number which is greater than the power of each family of incomparable elements in $Q^{a}$. If $\operatorname{cs}\left(P^{0}\right)$ $=n+1, n$ being finite, then it is shown that $P^{0}$ contains a coinitial subset $Q^{0}$ which is a degenerate ramified system with at most $n$ incomparable elements. Using combinatorial methods, a study is made of conditions under which $\operatorname{cs}\left(\prod_{a<\alpha} P^{a}\right) \leqq \aleph_{0}$, where $\Pi P^{a}$ is the cardinal product of the $P^{a}$, and $\operatorname{cs}\left(P^{a}\right) \leqq N_{0}$ for each $a$. For example, if $\alpha=2$, then $\operatorname{cs}\left(\prod_{a<\alpha} P^{a}\right) \leqq N_{0}$. Particular attention is focused on the case where each set $P^{a}$ is simply ordered. (Received July 15, 1952.)

623t. Casper Goffman: Lower semicontinuity of area functionals. I.

Frechet showed a number of years ago that a lower semicontinuous functional 
$u(x)$ on a metric space $S$, satisfying a certain condition $C$, has a unique extension to a lower semicontinuous $U(x)$ on the completion $\bar{S}$ of $S$, also satisfying $C$. If $S$ is the space of polyhedral mappings, properly metrized, and $u(x)$ is elementary area, then $\bar{S}$ consists of all continuous mappings and $U(x)$ is Lebesgue area. Now, elementary area is lower semicontinuous (with condition $C$ ) relative to other metrizations of the polyhedral mappings, and the Fréchet process may be applied to each of them. The long range objective is to make a thorough investigation of the possibilities. This first paper considers the nonparametric case. The elementary area $u(p)$ of a polyhedral function $p(x, y)$ is lower semicontinuous (with condition $C$ ) if the distance $d(p, q)$ is taken as $\iint|p(x, y)-q(x, y)| d x d y$. The functional $U(f)$, assured by Fréchet's Theorem, now has all summable functions as domain. It agrees with Lebesgue area for continuous functions. Much of the Rado-Tonelli theory for continuous functions is extended to this case, the salient ideas such as expressions of Goëcze, bounded variation, and absolute continuity in sense of Tonelli being appropriately modified. (Received July 11, 1952.)

\section{4t. Casper Goffman: One-one measurable transformations.}

This paper is a continuation of one given at the International Congress of Mathematicians (Cambridge, 1950) entitled Lusin's theorem for one-one measurable transformations. It is shown, for example, that if $I_{n}, I_{m}$ are $n$ - and $m$-dimensional unit cubes, $n<m$, and if $\left(f(x), f^{-1}(y)\right)$ is a one-one measurable transformation between them, then, for every $\epsilon>0$, there is a homeomorphism $\left(g(x), g^{-1}(y)\right)$ between $I_{n}$ and a subset of $I_{m}$ such that $f(x)=g(x)$ and $f^{-1}(y)=g^{-1}(y)$ on sets whose $n$-dimensional and $m$-dimensional measures, respectively, exceed $1-\epsilon$. The method of proof combines constructions similar to those used by Nöbeling and Besicovitch in surface area problems and theorems on the extension of homeomorphisms from given homeomorphisms on special zero-dimensional sets. The results of both papers will appear in Acta Mathematica. (Received July 11, 1952.)

\section{5t. A. W. Goodman: The rotation theorem for starlike univalent functions.}

Stroganoff [Trudy Mat. Inst. Steklova vol. 5 (1934) pp. 247-258] proved that among the functions $f(z), f(0)=0, f^{\prime}(0)=1$, regular, univalent and starlike in $|z|<1$, the extreme value of $\arg f^{\prime}\left(r e^{i \theta}\right)$ for fixed $r$ is assumed by $z /(1-z)^{2}$ at an appropriate point $z$. In this paper a short and elementary proof of the same result is obtained. The analogous problem for functions starlike in $|z|>1$ is discussed. (Received July 16, 1952.)

\section{P. R. Halmos, Günter Lumer, and J. J. Schäffer: Square roots of operators.}

The main purpose of this paper is to answer (negatively) the question: does every (bounded) invertible operator on a (complex) Hilbert space have a square root? Let $H$ be the Hilbert space of all square-integrable analytic functions on a bounded domain $D$, and let $A$ be the operator on $H$ defined by $(A x)(t)=t x(t)$ for all $t$ in $D$. It is shown that the spectrum of $A$ is the closure of $D$ and, more particularly, that if $\lambda \in D$, then $\lambda$ is not an approximate proper value of $A$ but $\bar{\lambda}$ is a simple proper value of $A^{*}$. From this in turn it is deduced that $A$ has a square root if and only if $D$ surrounds the origin, in any one of several, rigorously definable, senses of that phrase. It follows that if, for instance, $D$ is an annulus with center at the origin, then $A$ is invertible, 
but $A$ has no square root. The same methods solve the problem of $n$th roots for all $n \geqq 2$, and it turns out, in fact, that $A$ has an $n$th root if and only if it has a square root. (Received April 9, 1952.)

\section{J. M. Hammersley: Nelder's conjecture.}

Let $F(x)$ be a nondecreasing function satisfying $0 \leqq F(x) \leqq 1$. Then Nelder conjectured that $\int_{0}^{\infty} \int_{0}^{\infty}(x-y)^{2} e^{-(x+y)} d F(x) d F(y) \leqq 2 / e^{2}$, and that equality is attained only in the case $F(x)=0,1 / 2$, or 1 according as $x<0,0 \leqq x<2$, or $x \geqq 2$. The first part of this conjecture is proved by approximating the integral by a function of the saltuses of $F$, in case $F$ is a step function, and obtaining a contradiction on the magnitude of any saltus at zero if $F$ does not have the special form given above. The question of uniqueness remains open, although it is proved that the above $F$ is unique among the class of $F$ which have saltuses of commensurable magnitudes. Nelder's conjecture is an inequality in $[1,1]$ space, and has application to the theory of moment generating functions. (Received July 21, 1952.)

628. Fritz Herzog and George Piranian: Sets of radial continuity of analytic functions.

A point set $E$ on the unit circle $|z|=1$ is defined to be a set of radial continuity, provided there exists a function $f(z)$, regular in $|z|<1$, such that $\lim _{r \rightarrow 1} f\left(r e^{i \theta}\right)$ exists if and only if $e^{i \theta}$ is a point of $E$. It is proved that every set of type $F_{\sigma}$ is a set of radial continuity. The result is not the best possible. (Received July 1, 1952.)

\section{Fritz Herzog and George Piranian: Sets of convergence of} Taylor series. II.

The authors describe two types of constructions of Taylor series. The first type, essentially geometric in nature, is used to prove the following: (1) every denumerable set on the unit circle $C$ is the set of divergence of the Taylor series of a function which is schlicht and bounded in $|z|<1 ;(2)$ there exists a function, schlicht and bounded in $|z| \leqq 1$, whose Taylor series converges everywhere on $C$, but not uniformly on any arc of $C$; (3) if the set $M$ on $C$ is closed and nowhere dense, there exists a Taylor series which converges everywhere on $|z| \leqq 1$ to a function which is schlicht in $|z| \leqq 1$, regular on every arc of $C-M$, and unbounded on every open arc of $C$ that meets the set $M$. The second type of construction, of more arithmetic character, yields analogues to results (1) and (2), but with the condition of univalence replaced by continuity in $|z| \leqq 1$. (Received July 1,1952 .)

\section{0t. Edwin Hewitt: Linear functionals on almost periodic func- tions.}

Bochner's theorem on positive definite functions is generalized in the following way. Let $p(t)$ be an arbitrary complex-valued function defined for all real $t(t \in R)$ such that $\sum_{j, k=1}^{N} p\left(t_{j}-t_{k}\right) \xi_{j} \xi_{k} \geqq 0$ for all $t_{1}, \cdots, t_{N} \in R$ and all complex $\xi_{1}, \cdots, \xi_{N}$. Continuity or measurability of $p(t)$ is not assumed. For a bounded measurable set $T \subset R$, and any $\alpha \geqq \sup T-\inf T$, let $T_{\alpha}=\bigcup_{m=-\infty}^{+\infty}\{T+n \alpha\}$. Let $S$ be the smallest algebra of sets containing all $T_{\alpha}$. Then there exists a finitely additive, non-negative, bounded measure $\phi$ defined for all sets in $S$ such that $p(t)=\int_{-\infty}^{+\infty} e^{i t x} d \phi(x)$ for all $t \in R$. Subject to certain regularity conditions, $\phi$ is uniquely determined by this representation. The proof is obtained by considering the general form of a bounded linear functional $L$ on the space $\mathfrak{A}$ of continuous almost periodic functions on $R$, regarding $\mathfrak{A}$ as the space 
of all complex continuous functions on a certain compact group $b R$, applying the generalized Bochner theorem to $b R$, and finally representing $L$ as a finitely additive integral on $R$. As a corollary, it is proved that every positive definite function $p$ can be uniquely written as $p_{c}+p_{d}$, where $p_{c}$ and $p_{d}$ are positive definite, $p_{c}$ is continuous, and $p_{d}$ cannot be written as $q_{1}+q_{2}$ with $q_{1}, q_{2}$ positive definite and $q_{1}$ continuous and $\neq 0$. The relations between measures on $R$ and $b R$ are also studied. (Received April 9, 1952.)

\section{R. D. James: Generalized nth primitives.}

In a previous paper (Canadian Journal of Mathematics vol. 2 (1950) pp. 297-306) the author considered some of the properties of a Perron second integral ( $P^{2}$-integral), which, starting with a function, goes directly to its second primitive. The purpose of this paper is to define a $P^{2 m}$-integral for $m>1$. The definition is made directly and not in terms of $P^{2 r}$-integrals with $r<m$. It is shown that the $P^{2 m+2}$-integral includes and is more general than the $P^{2 m}$-integral. In a similar way a $P^{2 m+1}$-integral is defined with the same property of consistency as the $P^{2 m}$-integral. Moreover, the two scales fit together. The $P^{r+1}$-integral includes and is more general than the $P^{r}$-integral for $r>3$. It is also shown that the $P^{r+1}$-integral includes and is more general than the $C_{r} P$-integral defined by Burkill (Proc. London Math. Soc. (2) vol. 39 (1935) pp. 541552). (Received July 18, 1952.)

\section{2t. Fritz John: Elementary expressions for the derivatives of weak solutions of elliptic differential equations.}

Let $L$ be a linear elliptic differential operator of order $2 m$ with continuous coefficients defined in a domain $D$ in $E^{n}$. Let $\bar{L}$ be the adjoint operator of $L$. A function $u$ is said to be a weak solution of $\bar{L}(u)=B(x)$ if $\int[u L(v)-v B(x)] d x=0$ for every $v$ of class $C^{\infty}$ vanishing outside a compact subset of $D$. An explicit expression for the derivatives of a function agreeing almost everywhere with $u$ is given, using the method of spherical means (see Proceedings of the Symposium on Spectral Theory, Oklahoma, 1951, pp. 162 et seq.). This expression involves weighted integrals of $u$ over solid spheres with kernels formed from $B$ and the coefficients of $L$ by elementary operations. [Existence theorems for derivatives of weak solutions have been proved by F. Browder by a different method (Bull. Amer. Math. Soc. Abstract 58-2-162).] The construction given here yields estimates for the derivatives of $u$ in terms of $u$, which are related to but weaker than those given by K. O. Friedrichs. (Received June 26, 1952.)

633t. W. E. Johnson: Asymptotic solutions of a linear second order differential equation with two turning points.

The solutions of the differential equation $d^{2} y / d x^{2}-\left[\lambda^{2} \phi^{2}(x)-\chi(x)\right] y=0$ have been shown to be expressible asymptotically with regard to the parameter $\lambda$ in terms of Bessel functions throughout an interval containing at most one zero of $\phi^{2}(x)$. If a representation with elementary functions is to be used, the Stokes' phenomenon is encountered. Connection formulas must then be employed. When the interval considered contains two distinct zeros of $\phi^{2}(x)$, Bessel functions are no longer adequate for the representation of a solution by means of a single functional form. If Bessel functions are to be used, connection formulas must again be employed. In this paper it is shown however that with the use of Weber functions, a representation can be achieved for any solution of the differential equation over a real finite interval con- 
taining two simple zeros of $\phi^{2}(x)$. The forms are asymptotic with respect to the real positive parameter $\lambda$. (Received July 14, 1952.)

634t. Edward Kasner and John DeCicco: The Newtonian potential of a sphere of a Euclidean space $E_{N}$ embedded in a Euclidean universe $E_{n}$.

The two forces of attraction $F_{S}$ and $F_{V}$ exerted by a homogeneous material $(N-1)$ dimensional surface $S$ and $N$-dimensional volume $V$ of a sphere of radius $r$ and mass $M$ of a Euclidean space $E_{N}$ upon a particle $P$ of mass $m$ in $E_{N}$ at a distance $a$ from the center of the sphere, such that $E_{N}$ is embedded in a Euclidean universe $E_{n}$ of $n$ dimensions where $2 \leqq N \leqq n$, are studied. Either one of these two forces is directed along the line joining the position of the particle $P$ to the center of the sphere. If $N=n$, the results are extensions of the corresponding well known formulas for $n=2$ and 3 . If $2 \leqq N<n$, both $F_{S}$ and $F_{V}$ can be expressed in terms of hypergeometric functions. The corresponding fields of force are conservative and solenoidal. The Newtonian potential functions $U_{S}$ and $U_{V}$ are expressed in terms of hypergeometric functions. As a corollary, certain integrals of hypergeometric functions are evaluated. Finally some theorems of Gauss concerning the potential function due to the attraction of a circumference are extended. (Received June 19, 1952.)

635. W. S. Kimball: The uniqueness requirement of Jacobi's condition in the calculus of variations.

The integral $J=(1 / 2) \int y^{\prime 2} d x / a \cos a x$ whose extremals are $y=c \sin a x$ and $y=b$, between $0 \leqq a x \leqq \pi$ for a fixed $c>0$, has for its first family of extremals above an envelope, $y=c$, under variations in the family parameter $a$, where $c a$ is seen as the slope at the origin of the sinusoidal extremal arches. The two extremals $y=c \sin a^{\prime} x$, and $y=c \sin a^{\prime \prime} x$ that cross at each point $(x, y)$ under the envelope must have arguments that are supplementary angles $a^{\prime} x=(\pi / 2-r)$ and $a^{\prime \prime} x=(\pi / 2+r)$, where $a^{\prime \prime}$ $=$ constant gives the steeper sinusoidal extremal that touches the envelope $y=c$ before it reaches the end point $(x, y)$ where it meets the extremal $a^{\prime}=$ constant, which has not yet touched the envelope because $a^{\prime} x=\pi / 2-r$ is less than $\pi / 2$ of the argument $a x_{c}=\pi / 2$, that determines the conjugate points $x_{c}$ of contact with the envelope. For both extrema, $J_{\min }$ (contacting envelope) $=\left(c^{2} / 2\right) \sin a^{\prime \prime} x=J_{\min }$ (not touching envelope) $=\left(c^{2} / 2\right) \sin a^{\prime} x=c y / 2$. Thus $J_{\min }$ (contacting envelope) is not $J_{\min }$, unique although equal to the smallest values of $J$. But still Jacobi's condition is satisfied provided it is taken to prescribe that unique minima of $J$ cannot be provided along extremals having contact points with the envelope. (Received August 6, 1952.)

\section{6t. W. J. Klimczak: Differential operators of infinite order.}

Let $G(w)=\sum_{k=0}^{\infty} g_{k} w^{k}$ be an entire function of order less than or equal to $\sigma$ and type at most $\beta$ if the order equals $\sigma$. Let $D_{z}=\sum_{j=0}^{n} P_{j}(z) d^{n-i} / d z^{n-i}$. Then a necessary and sufficient condition that the operator $G\left(D_{s}\right)$ preserve holomorphism in any domain where the $P_{j}(z)(j=0,1, \cdots, n)$ are holomorphic is that $\sigma=1 / n$ and $\beta=0$. In a certain sense this result is the best possible. If the $P_{j}(z)(j=0,1, \cdots, n)$ are polynomials and if the degree of $P_{0}(z)$ is at most $n-1$, then $G\left(D_{z}\right) f(z)$ is an entire function provided $f(z)$ is an entire function and the order of $f(z)$ and $\sigma$ satisfy a certain inequality determined by an order relation depending upon the degrees of the polynomial coefficients. The emphasis is on the case $n=2$ and the extension to larger values of $n$ is indicated. The results generalize previous results of $\mathrm{H}$. Muggli, E. Hille, and M. K. Peabody and are sharp at least for $n=2$. (Received July 25,1952 .) 
637. E. G. Kundert: $A$ relation between poles and zeros of a simple meromorphic differential form.

It is well known that on a Riemann surface the degree of the divisor of a differential is always $=-\mathrm{X}$ (Euler's characteristic). For a simple differential form $\alpha$ in a complex manifold $M^{n}$ the following theorem holds, denoting by $C(\alpha)$ the zero-cohomology-class and by $S(\alpha)$ the polar-cohomology-class: $C(\alpha)$ can be expressed as a polynomial of degree $n$ of $S(\alpha)$ in the cohomology ring of $M^{n}: C(\alpha)=S^{n}(\alpha)$ $+\sum_{k=1}^{n} \Gamma_{n-k+1} \cdot S^{n-k}(\alpha)$; where the $\Gamma_{k}$ are the covariant Chern classes of $M^{n}$. The proof uses an association of a field of complex line elements to $\alpha$ and then one can use the theory developed in the author's paper in Ann. of Math. vol. 54 (1951). (Received August 11, 1952.)

638t. E. G. Kundert: $A$ simple method to calculate Chern's classes of an algebraic variety.

For various purposes it is important to have a method to calculate Chern's classes. The following formulas in the cohomology ring of an algebraic variety $V^{n}$ serve this purpose: $\Gamma_{1}=\Pi_{r}^{n}+\sum_{k=1}^{n} \Pi_{n-k+1} \Pi_{1}^{n-k} ; \Gamma_{k}=(1 /(k-1) !) \partial^{(k-1)} \Gamma_{1}\left(\Pi_{n}\right) / \partial \Pi_{n}, \Gamma_{k}$ being the contravariant Chern classes, $\Pi_{k}$ denoting the cohomology classes dual to the intersection cycle of a $(k+m-n-1)$-dimensional plane in $P^{m}$ with $V^{n}$, where $P^{m}$ is the lowestdimensional space in which $V^{n}$ can be imbedded without singularities. The proof of this formula consists in constructing a field of line elements on $V^{n}$ having $\Pi_{k}$ as secondary obstructions and using it as reference field for the Chern field in formula (19) and (20) in the Author's paper in Ann. of Math. vol. 54. (Received August 11, 1952.)

\section{9t. A. E. Livingston: A note on products of summability methods.}

Let $A$ and $B$ be regular summability methods for sequences, and define the method $A B$ by the $A$ transforms of the $B$ transforms of a given sequence. If, then, $s_{n} \rightarrow s(A)$ implies that $s_{n} \rightarrow s(A B)$, this will be indicated symbolically as $[A, B]$ is true. Let $A, B$, $(C, \alpha),(E, \gamma)$, and $(H, \mu)$ denote, respectively, the Abel, Borel, Cesaro (with parameter $\alpha>0$ ), Euler (with parameter $\gamma, 0<\gamma \leqq 1$ ), and Hausdorff (with kernel $\mu(x)$ ) summability methods. Otto Szász (On products of summability methods, Proceedings of the American Mathematical Society vol. 3 (1952) pp. 257-263) has shown that $[A,(C, \alpha)],[B,(C, \alpha)]$, and $[B,(E, \gamma)]$ are true. The author of this note shows that $[A,(H, \mu)]$ and $[B,(H, \mu)]$ are true, and these results include the results quoted above. Since $(H, \mu)(H, \sigma)=(H, \sigma)(H, \mu)$, it is trivial that $[(H, \mu)(H, \sigma)]$ is true. (Received July 24, 1952.)

\section{0t. A. E. Livingston: The space $H^{p}, 0<p<1$, is not normable.}

It has been conjectured that the space $H^{p}, 0<p<1$, is not normable (S. S. Walters, Remarks on the space $H^{p}$, Pacific Journal of Mathematics vol. 1 (1951) pp. 455-471). The author of this note shows that this conjecture is correct by proving the equivalent result: The space $H^{p}, 0<p<1$, contains no bounded convex neighborhood of the origin. (Received July 24, 1952.)

\section{1t. Dorothy Maharam: The structure of abstract integrals.}

In this paper an "abstract integral" is a linear, countably additive, order-preserving mapping $\phi$ of one function space $F$ in another, $F^{\prime}$. One example of such an "integral" is as follows. Let $X, Y$ be spaces in each of which a Borel field of "measurable" subsets and a $\sigma$-ideal of "null" sets are given, and suppose that in $X$ these are in 
fact given by a measure, $m$. Let $F$ be a space of (suitably restricted) "measurable" functions on $X \times Y$, and $F^{\prime}$ be the space of "measurable" functions on $Y$ (functions differing only on "null" sets being identified throughout); these functions need not be finite. Take $\phi(f)=g$ where $g(y)=\int f(x, y) d m(x)$. It is proved (under mild assumptions, mostly of countability, and with no use of norms) that every "abstract integral" $\phi$ is "isomorphic" to one of this type. Conditions are given under which $F$ will consist of (essentially) all "measurable" functions on some subset of $X \times Y$. (Received May 28, 1952.)

\section{Hazleton Mirkil: Translation-invariant function algebras over} compact groups. I.

All the major theorems of G. E. Silov's paper Homogeneous rings of functions (see Mathematical Reviews vol. 13 (1952) p. 139) are here extended to arbitrary compact groups; Silov did only the compact abelian case. Let $A=\{x\}$ be a commutative semi-simple symmetric banach algebra whose maximal-ideal-space is homeomorphic to a compact group $G=\{s\}$. (Multiplication is pointwise.) Call $A$ "left-homogeneous over $G^{n}$ if $A$ contains with every $x$ all the left-translates $s x$, in which case the left-translations $\{x \rightarrow s x\}$ are automatically continuous $A \rightarrow A$. Write $Q(G)$ for the characteristic algebra of $G, Q(G)=$ linear combinations of matrix coefficients of irreducible left representations of $G$. Theorem: a necessary and sufficient condition that all the mappings $\{s \rightarrow s x\}$ be continuous $G \rightarrow A$ is that $A$ contain $Q(G)$ and that $Q(G)$ be everywhere dense in $A$. The classical Peter-Weyl approximation theorem for continuous functions, clearly included as a special case, is used in the proof. Since the right-representations of $G$ define exactly the same $Q(G)$ as the leftrepresentations, it follows that for a left- and right-homogeneous $A$ the continuity of all $\{s \rightarrow s x\}$ implies the continuity of all $\{s \rightarrow x s\}$. (Received July 21,1952.)

643t. L. J. Mordell: The minima of some nonhomogeneous functions of two variables.

This paper contains a proof and some applications of the following: Theorem. Let $x_{0}, y_{0}$ be any real numbers. Then for certain functions, $f(x, y)$, there exist numbers $x, y$ such that $x \equiv x_{0}(\bmod 1), y \equiv y_{0}(\bmod 1)$, and $|f(x, y)| \leqq k \max (|f(1,0)|,|f(0,1)|$, $f(1, \pm 1)$, where $k$ is a number independent of $x_{0}, y_{0}$, depending only upon $f(x, y)$, and is really a constant defined by the existence of the inequality $|f(x, y)|$ $\leqq k|f(2 x, 2 y)|$ for all real $x, y$. The function $f(x, y)$ is supposed to be such that inter alia, the region defined by $|f(x, y)| \leqq 1$ is in general appearance similar to that defined by $\left|x^{8}+y^{8}\right| \leqq 1$ The paper has been accepted for publication in the Duke Mathematical Journal. (Received July 15, 1952.)

\section{4t. Zeev Nehari: Some inequalities in the theory of functions.}

Many of the inequalities of function theory and potential theory may be reduced to statements regarding the properties of harmonic domain functions with vanishing or constant boundary values. For the derivation of these inequalities a large number of different techniques and procedures have been used. It is the aim of this paper to show that many of the known inequalities of this type, and also others which are new, can be obtained as simple consequences of the classical minimum property of the Dirichlet integral. In addition to the resulting simplification, this method has the further advantage of being capable of generalization to a wide class of partial differential equations of elliptic type in two or more variables. While the ideal of using the 
positive-definite character of an integral as the source of function-theoretic inequalities is certainly not new, the present paper attempts to give a more or less systematic survey of the type of inequality obtainable in this way. (Received August $4,1952$.

645t. W. V. Parker and B. Evans Mitchell: Elementary divisors of certain matrices.

If $f(x)$ and $g(x)$ are polynomials and $P$ and $Q$ are matrices such that $(P-Q) f(P)$ $=(P-Q) g(Q)=0$, then $P$ and $Q$ have identical elementary divisors except for those associated with characteristic roots which are roots of either $f(x)=0$ or $g(x)=0$. It is shown that two theorems of Flanders (Proceedings of the American Mathematical Society vol. 2 (1951) pp. 871-874) follow as a consequence of this. (Received July 14, 1952.)

\section{6t. A. M. Peiser: An addition formula for spherical harmonics.}

Let $r$ denote a three-dimensional vector having magnitude $r$, polar angle $\theta$, and azimuth angle $\phi$. A spherical harmonic of $r$ is a function of the form $F_{n}^{m}(\boldsymbol{r})$ $=r^{n} P_{n}^{m}(\cos \theta) \exp (i m \phi),|m| \leqq n$. It is shown that if $r_{1}=r_{2}+r_{3}$, then $F_{n}^{m}\left(r_{1}\right)$ $=\sum_{k=0}^{n} \sum_{j-k}^{k} C_{n+m, j+k} F_{k}^{i}\left(\boldsymbol{r}_{2}\right) F_{n-k}^{m-i}\left(\boldsymbol{r}_{3}\right)$. Addition formulas of this type are useful in the evaluation of integrals involving products of spherical harmonics of different vectors. Such integrals have been encountered by the author (with S. Golden) in the quantum theory of chemical kinetics. (Received May 16, 1952.)

647t. L. L. Pennisi: A class of linear integro-differential boundary value problems.

This paper concerns a system of linear integro-differential equations of the first order (1) $\mathcal{L}_{i}(y) \equiv y_{i}^{\prime}(x)-\sum_{j=1}^{n} A_{i j}(x) y_{i}(x)-\int_{a}^{b} \sum_{j=1}^{n} C_{i j}(x, t) y_{j}(t) d t=0$, together with linear homogeneous boundary conditions of the form $(2) s_{i}(y)=\sum_{i=1}^{n}\left[M_{i j} y_{j}(a)+N_{i j} y_{j}(b)\right]$ $=0(i, j=1, \cdots, n)$. We shall give a new construction of the Green's matrix for the incompatible system (1), (2) which is an improvement over the method of Jonah (Bull. Amer. Math. Soc. Abstract 36-3-54) in that it is not necessary to introduce an arbitrary matrix. We also study a special class of integro-differential systems which is linear in a characteristic parameter, and termed definitely self-conjugate adjoint. For this system all the characteristic values are real, and the characteristic solutions may be chosen normed and orthogonal. Also, certain expansion theorems of this system, in terms of the characteristic solutions, are established by showing that our system is equivalent to an integral equation of the type to which the results of Reid (Trans. Amer. Math. Soc. vol. 36 (1931) pp. 475-485) are readily extensible. (Received July 2, 1952.)

\section{L. L. Pennisi: Construction of the Green's matrix for an in-} compatible integro-differential system.

This paper is concerned with the construction of the Green's matrix for an incompatible system of linear integro-differential equations of the first order (1) $\mathcal{L}_{i}(y) \equiv y_{i}^{\prime}(x)-\sum_{j=1}^{n} A_{i j}(x) y_{i}(x)-\int_{a}^{b} \sum_{j=1}^{n} C_{i j}(x, t) y_{j}(t) d t=0$, together with linear homogeneous boundary conditions of the form (2) $s_{i}(y)=\sum_{j=1}^{n}\left[M_{i j} y_{j}(a)+N_{i j} y_{j}(b)\right]$ $=0(i, j=1, \cdots, n)$. The system (1), (2) is called incompatible when it admits only the trivial solution $y(x) \equiv 0$. In an unpublished dissertation Jonah (Bull. Amer. Math. Soc. Abstract 36-3-54) has proved the existence of a Green's matrix for a boundary 
value problem associated with a system of integro-differential equations of the first order. We shall give a new construction of the Green's matrix for the incompatible system (1), (2) which is an improvement over the method given by Jonah in the sense that it is not necessary to introduce a matrix $B(x)$ which is to a large extent arbitrary and on which the construction depends. Our new construction is based upon the following result. Let $D$ be a matrix whose first row is $V_{\alpha \beta}, V_{\alpha \beta}$ and whose second row is $Z_{q p}, W_{q \beta}, p, q=1, \cdots, r ; \alpha=1, \cdots, r$. The homogeneous system (1), (2) is compatible if and only if $D$ is singular, where the elements of $D$ are given by welldefined integral functions. In fact, the number of linear independent solutions of (1) and (2) is precisely $r+R-\rho$, where $\rho$ is the rank of $D$. (Received July 11,1952.)

649. Pasquale Porcelli: Concerning uniform completeness of sums of reciprocals of linear functions.

Suppose $F[a, b], 0 \leqq a<b$, is the normed linear space of continuous (complexvalued) functions on $[a, b]$ with $\|f\|=\max _{x} \in[a, b]|f(x)|,\left\{k_{p}\right\}_{p=0}^{\infty}$ numbers distinct from one another and from 0 , and, if $S=\left\{\left(1+k_{p} x\right)^{-1}\right\}_{p-0}^{\infty}$ and $S \subset F[a, b], M[a, b]$ the closed linear manifold in $F[a, b]$ generated by $S$. If $k_{p} \notin[-\infty,-1]$, then each of the conditions (1) $1 \in M[0,1]$ and (2) $\sum\left\{1-\left|k_{p}^{-1}\left[2+k_{p}-2\left(1+k_{p}\right)^{1 / 2}\right]\right|\right\}=\infty$ is necessary and sufficient for $M[0,1]=F[0,1]$ (van Herk, Compositio Mathematica vol. 9 (1951) pp. 1-79, proved (2) sufficient if $\left\{k_{p}\right\}_{p=0}^{\infty}$ is an unbounded increasing sequence of positive numbers). If $R\left(k_{p}\right) \geqq 0$, then (i) $a>0$ implies $M[a, b]=F[a, b]$, (ii) $\epsilon>0$ and $M[0, \epsilon]=F[0, \epsilon]$ imply $M[0, b]=F[0, b]$ for $b>0$, and (iii) $\epsilon>0$ and $M[0,1]=F[0,1]$ imply there exist $\left\{t_{s}^{(n)}\right\}_{s=0}^{n}$ in $[0, \epsilon]$ and $f_{n}(x)=\sum_{p=0}^{n} c_{p}^{(n)}\left(1+k_{p} x\right)^{-1}$, such that $f_{n}\left(t_{s}^{(n)}\right)=1,\left\|f_{n}(x)-1\right\| \rightarrow 0$ as $n \rightarrow \infty$, in $[0, b], b>0$. If $f \in F[a, b], f$ real, and $\epsilon>0$, then there exist Stieltjes continued fractions $F, G$ with all poles in $[-\infty,-1]$ such that $\|f-(F-G)\|<\epsilon$. (Received July 9, 1952.)

\section{0t. M. H. Protter: An existence theorem for the Tricomi problem.}

Consider the equation $K(y) u_{x x}+u_{y y}=0$ where $K(y)$ is a monotone increasing function of $y$ with a continuous third derivative. Suppose $K(0)=0$ and $K^{\prime}(0) \neq 0$. Let $\Gamma$ be an arc in the upper half-plane with end points on the $x$-axis, and let $\gamma_{1}$ and $\gamma_{2}$ be the characteristics emanating from these end points which intersect; let $D$ denote the domain bounded by $\Gamma, \gamma_{1}$, and $\gamma_{2}$. The boundary value problem in which values are assigned on $\Gamma$ and $\gamma_{1}$ is solved for the domain $D$ with certain restrictions on the nature of $\Gamma$. The method of proof makes use of the fundamental solution of the equation $y u_{x x}+u_{y y}=0$ and a modified form of the results of Gellerstedt. A uniqueness theorem previously established by the author is essential. Since the Chaplygin equation is of the above form this answers in part a question raised by Frankl concerning the transonic flow out of a jet. (Received May 16, 1952.)

651. Gustave Rabson: $A$ note on the convergence of fourier series on compact groups.

Theorem: On any compact group not of dimension zero there is a continuous function with a non-absolutely convergent fourier series. It is a consequence of a formula of André Weil (L'intégration dans les groupes topologiques et ses applications, 1940, p. 87) that if a continuous central function $F$ on a compact group $G$ has a fourier series which converges to it absolutely at the identity, then the restriction of $F$ to any closed subgroup will also have this property. On any compact Lie group, not of dimension zero, there is a circular subgroup on which may be chosen a continuous 
even function whose fourier series diverges at the identity. This function may then be extended to a function on the group with the desired properties. If $G$ is not a $\mathrm{Lie}$ group it may be assumed to be connected and one may consider any nontrivial unitary representation with kernel $K$. Then $G / K$ is a Lie group of dimension at least one. We may then construct a function with the required properties on $G / K$. This induces a function on $G$, in the natural way, which also has the required properties. (Received June 9, 1952.)

\section{H. J. Reiter: On a property of Fourier transforms.}

Let $G$ be a locally compact abelian group. The following theorem is proved (for the notation, cf. Bull. Amer. Math. Soc. Abstracts 58-3-139, 140,141): if $\widehat{Z}_{I}$ is denumerable and the elements of $\widehat{Z}_{1}$ are independent, then the quotient-algebra $L^{1 / I}$ is isomorphic and isometric with the Banach algebra of all complex-valued functions $c(\widehat{x})$ defined and continuous on $\widehat{Z}_{I}$ and vanishing at infinity in case $\widehat{Z}_{I}$ is not compact, with norm $\|c(\widehat{x})\|=\max |c(\widehat{x})|, \widehat{x} \in \widehat{Z}_{1}$. In particular: for any such $c(\widehat{x})$ there is an $f(x) \in L^{1}$ such that the Fourier transform $\hat{f}(\hat{\bar{x}})=c(\widehat{x})$ for all $\widehat{x} \in \widehat{Z}_{I}$. The proof is based on the results announced in the abstracts mentioned above, and on the following lemma: under the conditions of the theorem, if $\phi$ is orthogonal to $I$, then $\phi$ has an absolutely convergent Fourier series. (Received July 21, 1951.)

\section{P. C. Rosenbloom: Linear partial differential equations of} parabolic type with constant coefficients.

Equations of the form $u_{t}=L u+f$ are studied, where $L$ is a linear differential operator of elliptic type of arbitrary even order with constant coefficients. By the use of the method of Ladyzhenskaya, Mat. Sbornik (1950), for estimating the fundamental solution, her uniqueness theorem is sharpened, and various properties of the Poisson integral representation of the solution are obtained. The approach to the solution of the equation $L u+f=0$ as $t \rightarrow+\infty$ is also studied, and some uniqueness theorems for such equations of elliptic type are obtained. (Received June 9, 1952.)

\section{4t. H. L. Royden: Some counterexamples in the classification of open Riemann surfaces.}

Recently Ahlfors and the author constructed a Riemann surface of hyperbolic type which possessed no nonconstant harmonic functions with a finite Dirichlet integral. In this paper we explore some of the consequences of this example and construct a Riemann surface on which the spaces $H D$ and $H B D$ have dimension $n$. A bounded Riemann surface is given which has no $H D$ functions on it which vanish on the relative boundary while it has a nonconstant $H D$ function on it whose normal derivative vanishes on the relative boundary. Finally, a Riemann surface is constructed which admits of a nonconstant bounded harmonic function, but no nonconstant harmonic functions with a finite Dirichlet integral. This shows that the classes $O_{H D}$ and $O_{H D}$ are distinct. (Received June 27, 1952.)

\footnotetext{
655. Walter Rudin: Analyticity, and the maximum modulus principle.

The author investigates the extent to which the maximum modulus principle characterizes the class of analytic functions. Let $K$ be a compact subset of the plane, bounded by a simple closed curve $C$, whose interior is $D$. An algebra $A$ of complexvalued functions continuous on $K$ is said to be a maximum modulus algebra on $K$ if
} 
for every $f \in A$ there is a point $z_{0} \in C$ such that $|f(z)| \leqq\left|f\left(z_{0}\right)\right|$ for every $z \in K$. The principal result of the paper is the following theorem: Suppose (1) $A$ is a maximum modulus algebra on $K$, (2) $A$ contains a nonconstant function $f$ which is analytic in $D$, and (3) $A$ contains a function $g$ which is schlicht on $K$ ( $g$ may or may not coincide with $f$ ); then every member of $A$ is analytic in $D$. The question whether (3) may be omitted from the hypotheses is not settled. However, if (1) is replaced by the stronger requirement that every $f \in A$ satisfy the maximum modulus principle with respect to every subdomain of $D$, then (3) may be omitted. (Received July 9,1952.)

656. James Sanders: The behaviour, near the boundary, of the Green's function of second-order partial differential equations. Preliminary report.

Let $P(x, y)$ be a point in a domain $D$ bounded by a contour $C$ with outer normal $n$. Let $G(P, Q)$ be the Green's function for $\Delta_{p} U \equiv U_{x x}+U_{y y}=0$, in $D$, with singularity $-\log r$ at $P=Q . r$ is the distance from $P$ to $Q$. Then, when $P=p$ on $C$, (1) $\partial G(p, Q) / \partial n_{p}=-2 \partial \log r / \partial n_{p}+F_{1}(p, Q)$. P. Lévy's result (Acta Math. vol. 42 (1920)) that $F_{1}(p, Q)$ is analytic for all $p \in C, Q \in C+D$, provided $C$ is analytic, is proved by a new direct method. It is further shown that if $C$ has continuous derivatives of order $m, F_{1}(p, Q)$ has continuous derivatives of order $(m-4)$ with respect to $p$ and of order $(m-3)$ with respect to $Q$ for $p \in C, Q \in D+C$. Next let $G(P, Q)$ be the Green's function, in $D$, of $U_{x x}+U_{y y}+a(x, y) U_{x}+b(x, y) U_{y}+c(x, y) U=0$ which has a fundamental solution $L(P, Q)=A(x, y) \log r+B(p, Q)$ : then, (2) $\partial G(p, Q) / \partial n_{p}$ $=-2 \partial L(p, Q) / \partial n_{p}-(\boldsymbol{\alpha} \cdot \boldsymbol{n})_{p} L(p, Q)+F_{2}(p, Q)$ where $\alpha$ is the vector with components $(a, b)$. If $C$ is three times continuously differentiable, $F_{2}(p, Q)$ is continuous for $p \in C$, $Q \in E+C$. The coefficients $a, b, c$ have been assumed analytic in $D+C$, for the sake of simplicity. (Received July 21, 1952.)

\section{J. A. Schatz: Representation of algebras with an involution.}

$A$ *-algebra $A$ is a complex Banach algebra with an identity element and a normpreserving conjugate linear anti-automorphism $a \rightarrow a^{*}$ of period two. A BIP-space is a complex Banach space with an inner product satisfying $(x+\lambda y, z)=(x, z)+\lambda(y, z)$, $(x, y)=(\overline{y, x}),(x, y)=0$ for all $y$ only if $x=0$, and $|(x, y)| \leqq\|x\|\|y\|$. A real functional on $A$ is a linear functional satisfying $f\left(a^{*}\right)=\overline{f(a)}$ for all $a \in A$. Using methods similar to those of Gelfand and Neumark for $C^{*}$-algebras [Matematiceskir Sbornik N.S. vol. 12 (1943) pp. 197-217], the following results can be obtained. Let $S$ be a set of selfadjoint elements of $A$ such that for each $s \in S$ there is a real functional $f_{c}$ with $\left\|f_{0}\right\|=1$ and $f_{s}(s)=\|s\|$. Then there is a BIP-space $X$ and a norm-reducing ${ }^{*}$-homomorphism $a \rightarrow T_{a}$ of $A$ onto an algebra of bounded operators on $X$ such that $\left\|T_{\diamond}\right\|=\|s\|$ for each $s \in S$. By choosing $S$ as the set of all self-adjoint elements of $A$, we obtain a *-isomorphism. If $A$ is a $B^{*}$-algebra, it is an immediate consequence that $a \rightarrow T_{a}$ is normpreserving. The Gelfand-Neumark result can be obtained by choosing $S$ appropriately and using results of Lax and Kaplansky. (Received June 26, 1952.)

658. Augusta L. Schurrer: On the location of the zeros of the derivative of rational functions of distance polynomials.

Let $R(x, y, z)=\prod_{k=0}^{a} F_{k}(x, y, z) / \prod_{k=q+1}^{p} F_{k}(x, y, z), 0 \leqq q \leqq p$, represent a rational function of the distance polynomials $F_{k}(x, y, z)=c_{k} \prod_{j-1}^{p h}\left[\left(x-x_{j k}\right)^{2}+\left(y-y_{j k}\right)^{2}\right.$ $\left.+\left(z-z_{j k}\right)^{2}\right]^{m j k}, m_{j k}>0$, where $\sum_{j=1}^{p} m_{j k}=n_{k}$, the "degree" of $F_{k}$. Let $R^{\prime}(x, y, z)$ $=|\nabla R|^{2} / 4 R$ be the "derivative" of $R$ in the sense of Nagy [Bull. Amer. Math. Soc. 
vol. 55 (1949) pp. 329-342]. It is shown that the following theorem due to Marden [Bull. Amer. Math. Soc. vol. 42 (1936) pp. 400-405] can be extended to the class of distance polynomials: If $F_{j}(x, y, z)$ has all of its zeros in the spherical region $\sigma_{j} S_{j}(v)$ $\leqq 0$ where $\sigma_{j}^{2} \equiv 1, S_{j}(v) \equiv\left(v-c_{j}\right)^{2}-r_{j}{ }^{2}, r_{j}>0$, and $v$ is the position vector of the point $P(x, y, z)$, it follows that every finite zero of $R^{\prime}$ either lies in one of the given spherical regions or in the region $\left(E(v) / \prod_{j=0}^{p} S_{j}(v)\right) \equiv \sum_{j=0}^{p} N N_{j} / S_{j}(v)$ $-\sum_{j=0, k=i+1}^{p} N_{j} N_{k} T_{j k} / S_{j}(v) S_{k}(v) \leqq 0$ bounded by the $p$-spherical $2 p$-ic surface $E(v)=0$. Using the preceding theorem it is possible to extend to the class of distance polynomials some well known results, due to Bôcher and Walsh, concerning the location of the zeros of the derivative of a rational function. A Gauss-Lucas, a Laguerre, and a Walsh cross-ratio type theorem are also developed. (Received June 20,1952.)

\section{9t. Harold Shniad: Convexity properties of integral means of} analytic functions.

For the class of functions analytic in an open circle $|z|<R$, the convexity properties of the circumferential means of order $t$ over circles $|z|=r<R$ are studied as functions of $r$. It is known that these means of order $t$ are convex for every analytic function providing $t$ is zero or of the form $2 / k$, where $k$ is a positive integer. The following results are obtained. The mean of order four of all analytic functions is convex. If $t$ is a number greater than 5.66, then there is an analytic function whose mean of order $t$ is not convex. (Received July 21, 1952.)

\section{Edward Silverman: An intrinsic inequality for Lebesgue area.}

The measure of a parallelogram is not less than the product of the distances between the two pairs of opposite sides. A corresponding inequality for Lebesgue area permits the generalization to area of certain properties of arc length. In particular if one considers only functions of finite Lebesgue area, then (i) Lebesgue area is lower semi-continuous with respect to point-wise convergence, and (ii) if one considers quasi-harmonic functions in a square (continuous and harmonic on each of a finite number of Jordan regions into which the square is decomposed) to be generalizations of quasilinear functions on an interval, then the connection between arc length and inscribed polygons carries through to Lebesgue area and inscribed quasi-harmonic functions. The basic inequality, suggested by Choquet, is another approach to a problem considered by Cesari and Fullerton. (Received July 21, 1952.)

661t. C. T. Taam: On linear differential equations with small perturbations.

Let $y(x)$ be any solution of $(1)\left(p(x) y^{\prime}\right)^{\prime}+q(x) y=0$, where $p(x)$ and $q(x)$ are complex-valued, defined for $x \geqq 1$, and $p(x)^{-1}$ and $q(x)$ belong to $L(1, R)$ for every large $R$. Let $g(x)$ be real-valued having a positive lower bound in $1 \leqq x<\infty$. If $\int_{1}^{x}|p(t)|^{-1} d t$ $=O(g(x))$ and if $g(x) q(x)$ belongs to $L(1, \infty)$, then it is proved in this paper that $y(x)=O(g(x))$ as $x \rightarrow \infty$. From this we prove that for some constants $A$ and $B, y(x)$ $-A x-B$ tends to zero as $x \rightarrow \infty$ provided there exists a constant $a$ such that $\left(p(x)^{-1}-a\right)$ and $x^{2} q(x)$ belong to $L(1, \infty)$. Given the weaker conditions $\left(p(x)^{-1}-a\right)$ and $x q(x)$ belong to $L(1, \infty)$ for some $a$, then for any constant $C$ there exists a solution $y^{*}(x)$ of (1) which tends to a limit $C$ as $x \rightarrow \infty$. On the other hand if $p(x)^{-1}$ and $q(x)$ belong to $L(1, \infty)$, then $y(x)$ tends to a limit as $x \rightarrow \infty$. These results are then extended to the general linear differential equations of the second order, of the third order, and with the help of the Phragmen-Lindelöf Theorems, to the complex domain. The main 
tool we use is an inequality due to R. Bellman (Duke Math. J. vol. 10 (1943) pp. 643647). (Received July 10, 1952.)

662. W. J. Thron: A class of meromorphic functions having the unit circle as a natural boundary.

In the continued fraction (1) $1+K\left(d_{n} z^{\alpha_{n}} / 1\right)$, where the $\alpha_{n}$ are positive integers, let (2) $\lim \left|d_{n}\right| 1 / \alpha_{n}=1$ and $\lim \alpha_{n}=\infty$. Then (1) converges to a meromorphic function $f(z)$ for all $|z|<1$. Set $h_{n}=\sum_{k=1}^{n+1} \alpha_{k}$. It is shown in this paper that if in addition to conditions (2) the continued fraction (1) satisfies $\lim \sup h_{n} / h_{n-1}=\infty$, then the function $f(z)$ to which (1) converges for $|z|<1$ has the unit circle as a natural boundary. The methods employed in the proof of this result are elaborations of procedures used by the author in a recent paper (Proc. Nat. Acad. Sci. U.S.A. vol. 36 (1950) pp. 51-54). (Received July 18, 1952.)

663. E. W. Titt, Roger Osborn, A. J. Kainen, W. C. Long, L. G. Worthington, and W. S. McCulley: On a theory of the linear second order partial differential equation with $n$ independent variables.

In contrast to the methods of Hadamard and M. Riesz, the method developed here is more closely allied to that of Volterra. This paper treats the normal hyperbolic interior, although the method has been applied to other problems in lower dimensional cases. Whereas Volterra integrates a potential along a line, here a potential is integrated over a hypersurface. For $n \geqq 4$ the initial potential is not the Green potential but an extension of a quantity used by Volterra outside the cone in $n=3$. In $n=2,3$, the initial potential is sometimes of the latter type and sometimes Green's. The $(n-1)$-tuple integral when reduced to a single integral is in the nature of a transform of the initial potential, the kernel of which apparently varies only with the dimension. It turns out that this kernel is related to Legendre and Tschebyscheff polynomials in the even and odd dimensions, respectively. This paper concludes with a discussion of the integrating factor which is obtained by applying a certain number of differential operators to the above mentioned transform. With further differential operations to be performed after the Green's theorem stage, the final formula may involve a potential distinct from the initial one. (Received July 21, 1952.)

\section{C. J. Titus and G. S. Young: An extension theorem. I.}

Let $P(x)$ and $Q(x)$ be real polynomials of degree $n$ and $n-1$ respectively where (a) the first coefficients are positive, (b) the roots are real and simple, and (c) the roots of $Q$ separate the roots of $P$. Let $f(t)$ be an $n+1$ times differentiable function of period $2 \pi$ such that $\left(f^{\prime}\right)^{2}+(f)^{2}>0$. Corresponding to the polynomials $P$ and $Q$ are polynomic differential operators. With these operators define $u=P[f], v=Q[f]$. There exists then a mapping $I: D \rightarrow E^{2}$, where $D$ is the closed unit disc, where $I$ is a light interior on Int $D$, where Bdy $D=\{(x, y) \mid x=\cos t, y=\sin t\}$ and where $I(x(t), y(t))$ $=(u, v), 0 \leqq t \leqq 2 \pi$; i.e., every curve of the form $(u, v)$ is the point-wise image of the boundary of a light interior mapping of the unit disc. (Received July 21,1952.)

\section{5t. C. J. Titus: An extension theorem. II.}

A class of matric differential operators is defined which yields an extension theorem similar to that in the previous abstract. The domain of definition in this case is not simply connected. (Received July $21,1952$. ) 


\section{6t. Leonard Tornheim: Derivatives of functions convex with respect to $n$-parameter families.}

Let $F$ be an $n$-parameter family of functions on the interval $a \leqq x \leqq b$; i.e., through every $n$ points, no two of which have the same abscissa, there is a unique member of $F$. Let $g$ be a function convex with respect to $F$; i.e., each member of $F$ intersects $g$ in at most $n$ points. If all functions of $F$ have $n-1$ continuous derivatives on $a<x<b$, then $g$ has $n-2$ derivatives. This generalizes an earlier result for $n=3$. (Received July 21, 1952.)

\section{7t. J. L. Ullman: The eigenvalues of a Toeplitz form.}

Let $f(\theta)$ be a Lebesgue integrable function satisfying $0<m \leqq f(\theta) \leqq M$, and having Fourier series $\sum_{-\infty}^{\infty} c_{n} z^{n}, z=\exp (i \theta), c_{n}=(1 / 2 \pi) \int_{0}^{2 \pi} z^{-n} f(\theta) d \theta$. Let $\lambda_{0}^{n}, \lambda_{1}^{n}, \cdots, \lambda_{n}^{n}$ be the eigenvalues arranged in ascending order of magnitude associated with the Toeplitz matrix $T_{n}(f)=\left|c_{q-p}\right|_{0}^{n}$, whose determinant is $D_{n}(f)$. Caratheodory (Rendiconti del Circolo Matematico di Palermo vol. 32 (1911)) proved that $\lim _{n \rightarrow \infty} \lambda_{n}^{n}=M$ and $\lim _{n \rightarrow \infty} \lambda_{0}^{n}=m$. His result was essentially broadened by Szegö (Math. Zeit. vol. 76 (1920)) who showed that the eigenvalues had the same distribution as the equally spaced ordinates of $f(\theta)$. This result was obtained by first determining the limit of $\left(D_{n}(f)\right)^{1 / n}$. The purpose of this paper is to obtain the distribution directly. Formal manipulations with infinite Toeplitz matrices $T(f)$ yield $(T(f))^{k}=T\left(f^{k}\right)$. It is shown that the following exact relationship holds, namely $\lim _{n \rightarrow \infty} \operatorname{Tr} .\left(T_{n}(f)\right)^{k} /(n+1)$ $=\lim _{n \rightarrow \infty} \operatorname{Tr} .\left(T_{n}\left(f^{k}\right)\right) /(n+1)=(1 / 2 \pi) \int_{0}^{2 \pi} f(\theta)^{k} d \theta$. Since Tr. $\left(T_{n}(f)\right)^{k}=\sum_{i}^{n}\left(\lambda_{0}^{n}\right)^{k}$, this implies that for any continuous $F(\lambda), \lim _{n \rightarrow \infty} \sum_{0}^{n}\left(F\left(\lambda_{i}^{n}\right)\right) /(n+1)=1 / 2 \pi \int_{0}^{2 \pi} F(f(\theta)) d \theta$, which is the mathematical statement of Szegö's result on distributions quoted above. (Received July 14, 1952.)

\section{8t. H. S. Wall: Concerning continuous continued fractions and} certain systems of Stieltjes integral equations.

The idea of continuous continued fraction (P. Adam, Revista Matematica vol. 11 (1951) pp. 180-190) is developed and use to prove (1) if each of $f$ and $g$ is a continuous fraction in an interval $[a, b]$ and $g$ of bounded variation in $[a, b],{ }_{0} I_{x}^{t}=\int_{x}^{t} f d g,{ }_{n} I_{x}^{t}$ $=\int_{x-1}^{t} I_{x}^{u} d g(u), n=1,2,3, \cdots$, then the series $\sum_{p-0}^{\infty} p I_{x}^{t}$ converges absolutely and uniformly for $a \leqq t, x \leqq b,(2)$ if each of $f_{1}, \cdots, f_{n}$ is a function which is continuous and of bounded variation in $[a, b]$, then there exists only one $n \times n$ matrix $A$ of functions such that $A_{i j}(x, t)=\delta_{i j}+\int_{x}^{i} A_{i+1, j}(s, t) d f_{i}(s), i=1,2, \cdots, n, a \leqq x, t \leqq b$, where $A_{n+1, j}$ $=A_{1, j}$. The matrix $A$ has the properties: $A(x, x)=I, A(x, t)=A(x, r) A(r, t)$. If $n$ is $2,\left[A_{11}(x, t) z+A_{12}(x, t)\right] /\left[A_{21}(x, t) z+A_{22}(x, t)\right]$ is the value of the continuous continued fraction $\phi_{x}^{2}\left(f_{1}, f_{2}, z\right)=\lim _{\delta \rightarrow 0}\left[b_{0}+K_{p=1}^{2 n+2} 1 / b_{p}\right]$, where $\delta$ is the norm of an ordered subdivision $\left\{x_{i}\right\}_{i=0}^{n+1}$ of $[x, t], b_{2 p}=f_{1}\left(x_{p+1}\right)-f_{1}\left(x_{p}\right), b_{2 p+1}=f_{2}\left(x_{p+1}\right)-f_{2}\left(x_{p}\right), p=0,1, \cdots$, $n, b_{2 n+2}=z$. (Received June 10,1952.)

669t. Alexander Weinstein: A new solution of Cauchy's problem for the wave equation and the equation of Poisson.

Let $k$ be a real parameter, $k \neq \neq-1,-3,-5, \cdots$, and let $x$ denote a system of $m$ variables $x_{1}, \cdots, x_{m}$. Let $u^{k}(x, t)$ satisfy the equation (1) $\Delta u^{k}=u_{t t}+k t^{-1} u_{t}^{k}$. For $k \geqq m-1$, the Cauchy problem for (1) with given $u^{k}(x, 0)$ and $u_{t}^{k}(x, 0)=0$ is solved by Poisson's method of mean values. For $k<m-1$, the Cauchy problem is reduced to the previous case by a repeated application of the recursive formulas $u_{t}^{h}=t u^{k+2}$, $(k+1) u^{k+2}(x, 0)=\Delta u^{k}(x, 0), u_{t}^{h+2}(x \cdot 0)=0$. The case $k=0$ corresponds to the wave 
equation which is solved by the new and simple formula $u^{0}(x, t)=2^{1-n} \Gamma^{-1}(n)$ - $\int_{0}^{t}\left(t^{2}-r^{2}\right)^{n-1} r u^{2 n}(x, r) d r+R_{n-1}$, where the integer $n$ satisfies the inequality $2 n \geqq m-1$. $u^{2 n}$ is the Poisson mean value of $\Delta^{n} u(x, 0)$ divided by $1 \cdot 3 \cdot 5 \cdots(2 n-1)$. The term $R_{n-1}$ is given by the formula $R_{n-1}=\Gamma^{-1}(2 n-1) t^{2 n-2} \Delta^{n-1} u^{0}(x, 0)+\cdots+u^{0}(x, 0)$. In the exceptional cases $k=-1,-3,-5, \cdots$, Cauchy's problem can be solved if and only if $u^{k}(x, 0)$ is a polyharmonic function. (Received June $\left.3,1952.\right)$

\section{John Wermer: On ideals in a class of commutative Banach algebras.}

It is known that the group algebra $L$ of a locally compact abelian group has the following properties: (1) $L$ is regular, (2) the elements of $L$ which lie in all maximal ideals except for a compact set are dense, (3) there exists a set of linear isometric operators $T$ on $L$ commuting with multiplication such that any hyperplane of $L$ invariant under all $T$ in the set is an ideal, (4) given $f \in L$ and $\epsilon>0$ there is some $g$ with $\|f g-f\|<\epsilon$. The author shows that in any commutative semi-simple Banach algebra satisfying (1), (2), (3), and (4), a closed ideal the boundary of whose hull has no nonempty perfect subsets equals the intersection of maximal ideals containing it. (The hull of an ideal is the set of maximal ideals containing it.) For group algebras similar results are known. (Received July 21, 1952.)

\section{L. C. Young: Discontinuous parametric surfaces and their $B L$ representations.}

Dirichlet integral methods [Young, Trans. Amer. Math. Soc. vol. 64 (1948) pp. 317-335] lead to admitting $B L$ representations [Nikodým, Fund. Math. vol. 21 (1933) pp. 129-150]. Closure as well as compactness are then attained. An essential tool is again harmonic interpolation (which does not increase the Dirichlet integral of a $B L$ function: this is little known). There is a corresponding representation theorem for generalized surfaces [Young, Bull. Soc. Math. France vol. 79 (1951) pp. 59-84]. (Received July 21, 1952.)

\section{Applied Mathematics}

\section{2t. H. W. Becker: On Richardson's compensation theorem.}

In the classic componsation theorem, the compensating EMF is $-I \Delta v$, where $I$ is the current originally in the variable branch $v$, acting in the circuit of present impedance. Expressing this abstractly, in the case of one source, if $\Delta_{v} Y_{z z}$ is the change in the circuit transfer admittance from source $s$ to receiver $z$ while the branch $v$ impedance increases by $\Delta v, f_{s v}$ and $f_{v s}$ are the transfer factors from $s$ to $v$ and $v$ to $z$, and $\theta$ and $\theta^{\prime}$ are the network determinants before and after the change, then $\Delta_{v} Y_{s s}$ $=\mp \Delta v \cdot f_{s v} f_{v z} / \theta \theta^{\prime}=\mp \Delta v \cdot Y_{s v} Y_{v^{\prime} z}=\mp \Delta v \cdot Y_{s v^{\prime}} Y_{v z}$, where the sign is - or + according as $v$ is effectively in series with $s$ and $z$ or in shunt with them. This shows that the theorem could also be phrased in terms of present current flowing in original circuit. Why not work with currents and admittances both taken at the same epoch? This was done, by D. E. Richardson, Electrical network calculations, New York, 1946, p. 150, in his "Equivalent EMF of Resistance Change Theorem." Succinctly, this is $\Delta_{v} Y_{\text {sa }}$ $=\mp\left\{Z_{v} \Delta v /\left(Z_{v}+\Delta v\right)\right\} f_{s v} f_{v z} / \theta^{2}=\mp\left(Z_{v} \| \Delta v\right) \cdot Y_{s v} Y_{v z}$, where $Z_{v}$ is the circuit impedance as originally seen from a point in $v$. Analogously, referring all data to the present epoch, $\Delta_{v} Y_{s z}=\mp\left\{Z_{v^{\prime}} \Delta v /\left(Z_{v^{\prime}}-\Delta v\right)\right\}_{s v} f_{v z} / \theta^{\prime 2}=\mp\left(Z_{v^{\prime}} \|^{\prime} \Delta v\right) Y_{s v^{\prime}} Y_{v^{\prime} z}$. Thus the price paid for simultaneity is the additional factor $\left(1 \pm \Delta v / Z_{v} \text { or } v^{\prime}\right)^{-1}$. (Received July 21,1952 .) 


\section{3t. John DeCicco: Magnetic fields of force in Euclidean space of $n$ dimensions.}

To extend the concept of magnetic fields of force to Euclidean space $E_{n}$ of $n$ dimensions, the following form of Coulomb's law is adopted. The force between two magnetic poles is directly proportional to their strengths and inversely proportional to the $(n-1)$ power of the distance between them. If a magnetized body is considered to be a Riemannian manifold $V_{N}$ of $N$ dimensions where $1 \leqq N \leqq n$ embedded in the Euclidean space $E_{n}$ together with an intensity of magnetization vector field defined in $V_{N}$, then the magnetic attraction produced by this body defines a conservative and solenoidal field of force. The corresponding potential function $U(x)$ is harmonic. The potential $U(x)$ due to a normally magnetized shell consisting of a $V_{n-1}$ together with an intensity of magnetization vector field normal to $V_{n-1}$ is proportional to the mean value of the intensity of magnetization relative to the solid angle subtended by $V_{n-1}$ at the given point $x$. Also considered are the relations between the potentials $U(x)$ and $V(x)$ due to a uniformly magnetized body $V_{N}$ and to a uniform charge of electricity throughout the region occupied by $V_{N}$. Finally applications to magnetized spheres in $E_{n}$ are given. (Received June 18, 1952.)

674. R. J. Duffin and D. H. Shaffer: On the modes of vibration of $a$ ring-shaped plate.

As is well known nodal lines cannot occur in the gravest mode of vibration of a membrane. The question has been raised by Weinstein whether the same statement is true for the vibrations of a thin, flat plate clamped on its boundary. A counterexample is considered here consisting of a plate clamped on two concentric circles whose radii are in the ratio $K$. For $K$ sufficiently large it is found that the mode of vibration having a single nodal diameter and no nodal circles has the lowest frequency. The critical value for $K$ is approximately 715 where it is found that the frequencies of the one nodal line mode and the mode with no nodal lines are equal. In the limit as $K$ becomes large the frequency of the mode with no nodal lines approaches a value which is approximately $7 \%$ greater than that of the limiting fundamental frequency. Received July 18, 1952.)

\section{5t. F. D. Faulkner: The problem of Oberth.}

The "Problem of Oberth" is the problem of choosing a thrust function in order to attain maximum altitude with a rocket. It was shown by B. Hamel (Zeitschrift fir Angewandte Mathematik und Mechanik vol. 7 (1927) pp. 451-452) that the problem has a solution. By changing the independent variables from the time to the distance along the path, and by a second elementary substitution, the proof of the existence of a solution is made simpler. It is shown that a single function is maximized at the upper end of the curve by the extremals. Also that extrema of point functions of distance along the path, velocity, and fuel consumption at the upper end of the curve can generally be obtained as the consequence of the maximization of that one function. (Received July 21, 1952.)

\section{Bernard Friedman: Characteristic roots of compound matrices.}

The use of direct products enables us to find the characteristic roots of certain matrices whose elements are also matrices. The following result is used: Let $A_{1}, A_{2}, \cdots, A_{k}$ be $n \times n$ matrices, $B_{1}, B_{2}, \cdots, B_{k}$ be $m \times m$ matrices, and let $C$ $=A_{1} \times B_{1}+\cdots+A_{k} \times B_{k}, A_{1} \times B_{1}$ representing the direct product of $A_{1}$ and $B_{1}$. If 
$A_{1}, \cdots, A_{h}$ have a common eigenvector $z$ such that $A_{1} z=\lambda_{1} z, \cdots, A_{k} z=\lambda_{k} z$, then every eigenvalue of $\lambda_{1} B_{1}+\cdots+\lambda_{k} B_{k}$ is an eigenvalue of $C$. We discuss also the characteristic roots of the $k n \times k n$ matrix which has the matrices $A_{1}, A_{2}, \cdots, A_{k}$ as the elements of its first row while in the $j$ th row the same matrices are repeated in cyclic order but shifted $j r$ places to the right, $r$ being a fixed integer. (Received July 18, 1952.)

\section{R. M. Hayes: Iterative methods of solving linear problems on Hilbert space.}

Many iterative procedures for solving linear problems, such as the Rayleigh-Ritz method, are allied to the method of "expanding subspaces." Thus, given a Hilbert space $A$ and a linear equation on that space, the equation is successively solved on each of a sequence $\left\{A_{k}\right\}$ of subspaces of $A$ satisfying the conditions (1) $A_{k}$ is con-

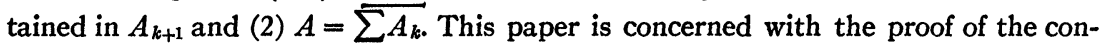
vergence of such iterative procedures for operators expressible as the sum of a positive definite operator and a completely continuous operator. In particular, one such procedure is the so-called "conjugate-gradient method," wherein the subspaces are successively determined by a sequence of conjugate vectors. Pertinent properties of the successive approximations in this method are considered; estimates on rates of convergence are given; and applications are shown. (Received July 15, 1952.)

678t. Stanley Katz and A. M. Peiser: A system of nonlinear partial differential equations arising in heat transfer.

Consider the differential equations $f(u) \partial u / \partial x=u-v=g(v) \partial v / \partial y$ in $x>0, y>0$, with boundary conditions $u(0+, y)=\phi(y), v(x, 0+)=\psi(x)$, where $\phi, \psi, f, g$ are continuous and $|f| \geqq a>0,|g| \geqq a>0$. It is shown that there exist unique solutions, continuous in $x \geqq 0, y \geqq 0$. For the corresponding difference equations $f\left(U_{m, n}\right)\left(U_{m+1, n}\right.$ $\left.-U_{m, n}\right) / h=U_{m, n}-V_{m, n}=g\left(V_{m, n}\right) \cdot\left(V_{m, n+1}-V_{m, n}\right) / k$ with boundary conditions $U_{0, n}$ $=\phi(n k), V_{m, 0}=\psi(m h)$, it is proved that $U_{m, n}$ and $V_{m, n}$ converge to $u(x, y)$ and $v(x, y)$ respectively as the interval lengths $h, k$ approach zero. In the linear case, $-f=g \equiv 1$, $\phi \equiv 1, \psi \equiv 0$, error estimates are obtained for $u(x, y)-U_{m, n}$ and for $v(x, y)-V_{m, n}$. (Received May 16, 1952.)

679. Franco Modigliani and F. E. Hohn: Production planning over time: An example of nonlinear programming.

This paper considers the problem of scheduling production of a given commodity over a sequence of equal, successive periods of time in such a way as to meet initially known demands in these periods while incurring the lowest possible total cost. The problem is solved for the case in which marginal cost in each period is the same, nonnegative, strictly increasing, differentiable function of production in that period only. Economic implications of the results are discussed. (Received July 21, 1952.)

680t. T. S. Motzkin and I. J. Schoenberg: On the relaxation method for linear inequalities.

Let $H_{i}: \sum_{i=1}^{n} a_{i j} x_{j}+b_{i} \geqq 0(i=1, \cdots, m)$ be a set of closed half-spaces of $E_{n}$ such that $A=\widehat{\bigcap} H_{i}$ is nonvoid. Let $p_{0} \notin A$, and let $H_{j}$ be a half-space such that dist $\left(p_{0}, H_{j}\right)=\max _{i} \operatorname{dist}\left(p_{0}, H_{i}\right)$. Let $\left|p_{0}-q\right|=\operatorname{dist}\left(p_{0}, H_{j}\right), q \in H_{j}$. Let $0<\lambda \leqq 2$ and let $p_{1}=p_{0}+\lambda\left(q-p_{0}\right)$. If $p_{1} \notin A$ we can repeat the process on $p_{1}$, with the same $\lambda$, obtaining the point $p_{2}$. If $p_{2} \notin A$ we obtain $p_{3}$ and so forth. S. Agmon has shown in a 
paper to appear in Ann. of Math. that if $0<\lambda<2$, then this process either terminates with a $p_{k} \in A$, or else it results in an infinite sequence $[p n]$ which converges to a point on the boundary of $A$. A new geometric approach to this problem is given which also includes the case of $\lambda=2$. In particular it is shown that if $\lambda=2$ and $A$ is $n$-dimensional, then the process always terminates. (Received July 21, 1952.)

\section{1t. L. E. Payne: Axially symmetric problems in elasticity. I. The punch problem.}

The usual procedure for solving the axially-symmetric punch problem amounts to finding a biharmonic function which satisfies a pair of dual integral equations. By formulating the problem in a manner analogous to that introduced by A. E. Green [On Boussinesq's problem and penny-shaped cracks, Proc. Cambridge Philos. Soc. vol. 45 (1947) pp. 252-257] one can reduce the problem to a determination of an axiallysymmetric potential function satisfying certain boundary conditions. By formulating the problem in this way rather than in terms of a biharmonic function, curvilinear coordinates may be more conveniently introduced and consequently the problem is in many cases greatly simplified. It is found that in most cases the necessity of solving a pair of dual integral equations can be eliminated. In particular, the solution to the spherical punch problem, which was approximated by $\mathrm{F}$. Chong [Indentation of a semi-infinite medium by an axially symmetric rigid punch, Iowa State College Journal of Science vol. 26 (1952) pp. 1-15], can now be obtained in closed form with little difficulty. (Received July 21, 1952.)

\section{Mary H. Payne: A generalization of Laplace's equation using spinors.}

The solutions of Laplacers equation in spherical coordinates are compared with the solutions of its analogue in spinor coordinates. All solutions of Laplace's equation are solutions of the spinor equation. There are, however, solutions of the spinor equation which are not solutions of Laplace's equation. Among these additional solutions are found functions which might be called "harmonic functions of half integral order," as well as some extra "harmonic" functions of integral order. The spinor coordinates are $S_{1}=(2 r)^{1 / 2} \cos (\theta / 2) e^{i(\phi+\psi) / 2}, S_{2}=(2 r)^{1 / 2} \sin (\theta / 2) e^{i(\psi-\phi) / 2}$, and their complex conjugates. (Received July 18,1952 .)

\section{Charles Saltzer: The second fundamental theorem of electrical networks.}

If a lumped-parameter linear network is represented by branches consisting of admittances in parallel with constant current sources, the mesh method can be replaced by the nodal method. The matrix equations of Kron are established rigorously by topological or graph-theoretical considerations, and conditions for the existence and uniqueness of the solution of the nodal equations are given. (Received July 23, 1952.)

\section{4t. P. A. Samuelson: The exact order of convergence of an ac- celerated iteration.}

For any sufficiently differentiable iteration, $X_{n+1}=g\left(X_{n}\right)$, with an isolated stationary point $A=g(A)$, the newly-defined accelerated iteration, $X_{n+1}=F\left(X_{n}\right)$, where $F(X)=\left[X g(g(X))-g(X)^{2}\right] /[X+g(g(X))-2 g(X)]$ has the same stationary point $A$ and has local derivatives there with the following properties: (a) for $g^{\prime}(A) \neq 1$, the 
first $r-1$ derivatives vanish and $F^{(r)}(A)=g^{(r)}(A) g^{\prime}(A)\left[g^{\prime}(A)^{r-1}-1\right] /\left[g^{\prime}(A)-1\right]^{2}$; (b) for $g^{\prime}(A)=1, F^{\prime}(A)=1-r^{-1}$; and (c) for $g^{\prime}(A)=0$, the first $2 r-2$ derivatives of $F$ vanish and $F^{(2 r-1)}(A)=-C_{2 r, r} g^{(r)}(A)^{2} / 2 r$-where in every case $r$ represents the first nonvanishing higher derivative of $g$ at $A$. Hence, it follows that $F$ is always a locally stable iteration and if, as in case (b), it does not already converge more rapidly than any locally linear iteration, the accelerated iteration, $F_{2}\left(X_{n}\right)$ $=\left[X_{n} F\left(F\left(X_{n}\right)\right)-F\left(X_{n}\right)^{2}\right] /\left[X_{n}+F\left(F\left(X_{n}\right)\right)-2 F\left(X_{n}\right)\right]$, will surely do so. Unless $g^{\prime}(A)$ $=1$, it will usually not pay to compute $F_{2}$, just as it will usually not pay to compute $F$ in the case where $g^{\prime}(A)=0$, since in this case $g$ already has convergence of higher than the first order and since $g\left(g\left(X_{n}\right)\right)$, representing two whirls of $g$, will have more vanishing derivatives than will $F$, their number being $r^{2}-1>2 r-2$. (Received May 16, 1952.)

\section{Domina E. Spencer: A Riemannian space for astronomy.}

The interpretation of astronomical data depends on the geometry that is postulated for cosmic space. All the data are obtained at essentially one point in the universe and give no sure indication of relationships at stellar distances. The practical interpretation of the data has ordinarily been made on the assumption of a euclidean metric, though general relativity employs a Riemannian metric with $R$ of the order of $10^{10}$ lightyears. A study is made of the possibilities of a non-euclidean world, limited by the assumption that space is locally euclidean and isotropic. Masses, densities, and luminous outputs of stars remain unchanged. An investigation of data on binary stars and cepheids shows that if the space constant $R$ of a Riemannian universe is 5 lightyears, Einstein's relativity is unnecessary. Time and space may be regarded as separate and distinct, a universal time scale can be established, velocity of light is constant with respect to the source, and the "expanding" universe ceases to expand. (Received July 9, 1952.)

\section{6t. M. L. Stein: A Rayleigh-Ritz-like procedure for minimizing} integrals. Preliminary report.

In this paper a procedure is presented for constructing the minimum of an integral $I(y)=\int_{a}^{b} f\left(x, y, y^{\prime}\right) d x$ among the arcs $y \equiv\left(y_{1}, \cdots, y_{n}\right)$ such that each $y(x)$ $(i=1,2, \cdots, n)$ is of class $c^{1}$ on $a \leqq x \leqq b$ and satisfies certain fixed end conditions. In the algorithm it is assumed that the $i$ th estimate of the minimum $y^{i}$ has been found. The integral $I$ is then replaced by a suitable quadratic integral $I^{*}\left(y^{i}\right)$ and the Rayleigh-Ritz method is applied to $I^{*}$ in order to obtain a variation $\eta^{i}$. The succeeding approximation is defined to be $y^{i+1}=y^{i}+\eta^{i}$ and the process is repeated. Conditions are given under which the sequence $\left[y^{i}\right]$ converges uniformly to a strong relative minimum. The minimum is expressed in the form $y_{0}+\sum_{i} a_{i} z^{i}$ where $y^{0}$ is an initial estimate satisfying the end conditions, $\left[z^{i}\right]$ is a set of basic functions in a suitable Hilbert space, and $a_{i}$ are scalars. (Received June 9, 1952.)

\section{Geometry}

\section{7t. Eugenio Calabi and M. A. Rosenlicht: Complex analytic manifolds without countable base.}

Any Riemann surface is known to have a countable base for its open sets. In this paper we verify a conjecture of Bochner that there exist complex manifolds of complex dimension $>1$ that are non second-countable by explicitly constructing a large 
class of such manifolds. Some basic topological properties of these manifolds are developed. (Received June 23, 1952.)

\section{8t. R. M. Conkling and D. O. Ellis: Metric $\Delta$-lattices.}

A lattice $L$ with a third operation which commutes and distributes over join and meet is called a $\Delta$-lattice. If $L$ is normed, and $\Delta$-translations are isometries, $L$ is called a metric $\Delta$-lattice. It is shown in this paper that a normed lattice admits a third operation to form an associative metric $\Delta$-lattice if and only if it is isometrically lattice isomorphic to the metric lattice of a certain type of lattice of isometric endomorphisms on itself. It is also shown that the class of reduced Gaussian semigroups is algebraically equivalent to a class of suitably restricted associative metric $\Delta$-lattices. (Received May 26, 1952.)

689t. David Gale: On inscribing $n$-dimensional sets in a regular $n$ simplex.

It is proved that every set of diameter 1 in $n$-space can be inscribed in a regular $n$-simplex of side $s<(n(n+1) / 2)^{1 / 2}$ but in no smaller simplex. Among the consequences of this result is the fact that every plane set of diameter 1 is the union of 3 sets each of diameter not greater than $3^{1 / 2} / 2$. (Received June 13,1952.)

\section{0t. C. C. Hsiung: Some curves in Riemannian space.}

In a Riemannian space $R_{n}$ of $n$ dimensions, let $\bar{C}$ be a neighboring curve corresponding to a given curve $C$ such that the corresponding point $\bar{P}$ on $\bar{C}$ of any point $P$ on $C$ is on the unit 2-normal vector $\xi_{(2)}$ of $C$ at $P$ with an infinitesimal distance $\epsilon$ from $P$. Let $\bar{\xi}_{(2)}^{\prime}$ be the vector obtained by displacing the unit 2-normal vector $\bar{\xi}_{(2)}$ of $\bar{C}$ at $\bar{P}$ parallelly back to $P$ by the infinitesimal displacement along $\xi_{(2)} . n-2$ necessary and sufficient conditions for $\bar{\xi}_{(2)}^{\prime}$ to coincide with $\xi_{(2)}$ in the first order of $\epsilon$ are obtained in terms of the $n$ curvatures of $C$ at $P$ and some other invariants associated with $C$ and $R_{n}$ at $P$. This result can be considered as an extension of the following one: In a Euclidean three-dimensional space if there exists a curve $\bar{C}$ such that its binormals are also the binormals of a given curve $C$, then $C$ is a plane curve. (Received July 22, 1952.)

691. W. R. Hutcherson and S. T. Gormsen: Maps of certain algebraic curves invariant under cyclic involutions of periods three, five, and seven.

The authors contrast the mapping of general plane algebraic curves of orders 3, 5, 7 [Mlle J. Dessart, Sur les surfaces representant l'involution engendree par une homographie de periode cinq du plan, Mem. de Liege, vol. 16, no. 2] invariant under transformation $T, x_{1}^{\prime}: x_{2}^{\prime}: x_{3}^{\prime}=x_{1}: \epsilon x_{2}: \epsilon^{2} x_{3}$ into spaces $3,4,5$ getting surfaces of orders $3,5,7$, where $p=3,5,7$ respectively, and $\epsilon^{p}=1$. Certain general invariant space curves in $S_{3}$ and supported by given algebraic surfaces of order $7,3,3$ are mapped into spaces of orders $7,11,17$. The involution of period three on the seventh degree surface has two isolated points of coincidence as well as a line of invariant points. The isolated points are found to be perfect. The imperfect point $P_{3}$ of $I_{5}$ contained on the cubic surface [W. R. Hutcherson, Maps of certain cyclic involutions on two dimensional carriers, Bull. Amer. Math. Soc. vol. 37 (1931) pp. 759-765] is more accurately treated and found to have a perfect point in the second order neighborhood instead of the third. The neighborhood of each of the three branch points (images of points of 
coincidence in the plane containing $I_{7}$ ) on the surface in $S_{5}$ are investigated. (Received July 11, 1952.)

692. W. R. Hutcherson and J. C. Morelock: Invariants with respect to special projective transformations.

A method for finding homogeneous polynomials invariant under an involutory transformation [W. R. Hutcherson, Fifth order neighborhood of an involution of period thirteen, Bull. Amer. Math. Soc. vol. 57 (1951) p. 484] is discovered and exhibited. The total number of terms in the general polynomial is synthetically built up by using the $p$ (prime number) classifications as component parts [J. C. Morelock, $A n$ algebraic formula, Amer. Math. Monthly vol. 57 (1950) p. 703]. The I.B.M. machine was used to obtain data for classifying perfect points in the infinitesimal neighborhoods of an imperfect point, and along the two invariant directions from this point, which is located on a certain fourth degree algebraic surface. (Received July 11, 1952.)

\section{3t. Edward Kasner and John DeCicco: The Fourier heat equa- tion in Riemannian space.}

If a body which occupies an $n$-dimensional region of Riemannian space $V_{n}$ and which is capable of absorbing heat is heated by conduction in any manner, the resulting situation may be visualized as a flow of heat. It is deduced by the divergence theorem in $V_{n}$ that the temperature $U$ at a position $x$ within the body and at a time $t$ obeys the Fourier heat equation: $\operatorname{div}(k \partial u / \partial x)=c \rho \partial u / \partial t$, where $k$ is the conductivity, $c$ the specific heat, and $\rho$ the density of the body. If $k, c, \rho$ are all constant so that the diffusivity $a^{2}=k / c \rho$ is constant, the Fourier heat equation is: $g^{-1} \partial\left(g^{1 / 2} g^{i j} \partial U / \partial x^{j}\right) / \partial x^{i}$ $=\left(1 / \alpha^{2}\right) \partial U / \partial t$. If $\nu(x ; t)$ obeys this latter equation, the heat hypersurfaces are defined by $U=\nu(x ; t)$, where $U$ and $t$ are constants. By means of homothetic representations of Riemannian manifolds, it is found that the only heat families which consist of exactly $\infty^{1}$ hypersurfaces are given by $f(x)=$ const., where $f$ is a solution of either the Laplace equation, or a special form of the Poisson equation, or the HelmholtzPockels equation. (Received June 19, 1952.)

694. L. M. Kelly and Leo Lapidus: The geometry of L-metrized spaces.

If with each two elements of a set $S$ is associated an element $\rho(a, b)$ of a lattice $L$ with an $O$ such that (1) $\rho(a, b)=\rho(b, a) ;(2) \rho(a, b)=0$ iff $a=b$; (3) $\rho(a, b) \cup_{\rho}(b, c)$ $\supset \rho(a, c)$, then $\rho(a, b)$ is called the distance from $a$ to $b$ and $S$ an $L$-metrized space. When $S \equiv L, \rho(a, b)$ is written $a \# b$, \# is called a metric operation and $L$ autometrized. The case in which $S \equiv L \equiv$ a Boolean algebra with $a \# b=a b^{\prime} \cup_{a^{\prime} b}$ has been called a Boolean geometry and studied by Ellis, Blumenthal, and Elliott. This paper is a study of the more general situation described above together with the special case of autometrized Brouwerian algebras (logics) with $a \# b=(a-b) \cup(b-a)$. Typical theorems: (1) A Brouwerian algebra is a Boolean algebra if and only if it admits a metric group operation; (2) A finite distributive lattice is a Boolean algebra iff it admits a metric operation such that the resulting space contains no isosceles triangles. (3) The congruence order of a Brouwerian chain with respect to the class of $L$-metrized spaces is 4. (4) The congruence order of a Brouwerian space with respect to the class of $L$-metrized spaces is three iff it is a Boolean geometry. (5) If \# is a metric group operation in a lattice $L$ and metric and lattice betweenness coincide, then $L$ is a Boolean geometry. (Received July 21, 1952.) 


\section{T. Minagawa and Tibor Radó: Infinitesimal rigidity of surfaces.}

Let $S$ denote a regular piece of surface whose boundary consists of a finite number of simple closed curves. Let $b$ be a portion of the boundary, where $b$ may be a subarc of a boundary curve or it may consist of one or more of the boundary curves. Then $S$ is termed IRb (infinitesimally rigid for $b$ kept fixed point-wise) if the identity is the only infinitesimal deformation of $S$ which leaves $b$ fixed point-wise. The purpose of this study is to establish the IR $b$ property for various categories of surfaces whose Gauss curvature has constant sign (positive, negative, or zero), including cases where the surface has corners. This study is being carried out under a research grant from the Office of Ordnance Research, U. S. Army. (Received July 17, 1952.)

\section{Ernest Mitchell: On Kasner's Neo-Pythagorean triangles.}

Kasner has called Neo-Pythagorean that class of triangles whose defining property is that the sum of the squares of the sides shall be zero. Such a triangle must of necessity lie in the complex plane, being in toto or at least partim imaginary. The object of this paper is to give this class of triangles a real representation, carrying along with it for the sake of analogy the Pythagorean class immersed in the complex plane. The method of representation is that of Laguerre. The two classes of triangles assume a quasi-conjugate relationship as exhibited by their "pictures" (Study). (Received July 10, 1952.)

\section{Don Mittleman: Trajectories of trihedral elements.}

Starting with the newtonian equations of translation of the center of mass and rotation about the center of mass of a rigid body of finite dimensions in a positional field of force, approximations to the equations are obtained when the maximum diameter of the body is small. These equations are called the equations of motion of a microscopic body. Also, limiting forms of the original equations are obtained when the maximum diameter approaches zero. These equations are called the equations of motion of a trihedral-particle. This latter result is dependent on the limits of the ratios of the principal moments of inertia. A trihedral element consists of a point (the center of mass of the body) and the three mutually orthogonal directions through the point (the principal axes of inertia). A trajectorial series is $\infty^{\prime}$ trihedral elements associated with the motion of a body for a given set of initial conditions. The differential equations for the trihedral series associated with all motions of a rigid body, all motions of a microscopic body, and all motions of a trihedral-particle are obtained. (Received June 12, 1952.)

598. T. S. Motzkin and I. J. Schoenberg: On Fejér sets in linear and spherical spaces.

Let $M$ be a metric space, $A \subset M$. We denote by $F(A)$, called the Fejer set of $A$, the set of those points $p$ with the property that there is no point $p^{\prime}$ such that $p x>p^{\prime} x$ for all $x \in A$. If $M$ is a real, inner-product space it is shown that $F(A)=K(A)$, where $K(A)$ is the intersection of all closed half-spaces containing $A$. In particular for three points $p_{1}, p_{2}, p_{3}$ we have (1) $F\left(p_{1}, p_{2}, p_{3}\right)=\Delta\left(p_{1}, p_{2}, p_{3}\right)$, where $\Delta\left(p_{1}, p_{2}, p_{3}\right)$ is the triangle of vertices $p_{1}, p_{2}, p_{3}$. Let now $M$ be a real Banach space $B$ which may also be finite-dimensional. It is shown: 1 . If $B$ is 2-dimensional, then (1) holds for any three points if and only if its metric is strictly convex. 2. If the dimension of $B$ 
is $\geqq 3$, then (1) holds if and only if $B$ is an inner product space. Also Fejer sets in spherical spaces are determined. (Received July 21, 1952.)

\section{9t. T. K. Pan: Relative first curvature and relative parallelism.}

Let a Riemannian space $V_{n}$ with positive definite first fundamental form $g_{i j} d x^{i} d x^{i}$ be immersed in a Riemannian space $V_{n+1}$. Let $\lambda$ be unit vectors in $V_{n+1}$, which are not in $V_{n}$ except in its asymptotic directions and whose contravariant components $\lambda^{d}$ at a point $P$ in $V_{n}$ are analytic functions of $x^{i}$ and $d x^{i}$ at $P$. The totality of these vectors is called a $\lambda$-congruence. Let $t$ be the unit tangent vector at a point $P$ of a curve $C$ in $V_{n}$. Let $N$ be a unit vector at $P$ satisfying the conditions: (1) linearly dependent on $t$ and $\lambda$ at $P,(2)$ orthogonal to $t$. When the first curvature vector at $P$ of $C$ in $V_{n}$ is decomposed along $N$ and a direction in $V_{n}$, the magnitude of the tangential component is called the first curvature at $P$ of $C$ relative to the $\lambda$-congruence. A curve, at each point of which the relative first curvature is zero, is called a pseudogeodesic in $V_{n}$. Pseudo-geodesics in $V_{n}$ are relative auto-parallel curves in $V_{n}$ according to a definition of relative parallelism, which is a generalization of the parallelism of Levi-Civita. Geometric interpretations of the relative curvature of a curve and properties of relative parallel displacement of vectors with respect to a curve in $V_{n}$ are studied. (Received July 21, 1952.)

\section{LOGIC AND Foundations}

\section{R. L. Davis: Structures of finite relations.}

Carnap pointed out recently that the number of structures (isomorphism classes) of a dyadic relation on a finite set was not known, adding that such information would have important applications in deductive and inductive logic and certain branches of science (Logical foundations of probability, Chicago, 1950, p. 124). If $R_{n}$ is the set of all relations of some particular kind defined on a set of $n$ elements, there is a group of permutations of $R_{n}$ which is isomorphic to the symmetric group, $\mathfrak{S}_{n}$. The orbits (sets of transitivity) under this group are just the isomorphism classes in $R_{n}$. Hence, by a well known proposition of group theory, the number of structures (i.e., of orbits) is $(1 / n !) \sum_{\pi \epsilon} f\left(t_{\pi}\right)$, where $f\left(t_{\pi}\right)$ is the number of relations fixed under the transformation $t_{\pi}$ corresponding to an element $\pi$ of $\mathfrak{S}_{n}$. This reduces the problem to that of counting the number of relations so fixed. Employing various combinatorial arguments, general formulas for the number of structures are derived in each of the following kinds of relations (all but (i) dyadic): (i) arbitrary $m$-adic; (ii) reflexive, or irreflexive; (iii) symmetric; (iv) irreflexive-symmetric; (v) asymmetric, and (vi) functions. (Received June 4, 1952.)

701. J. C. C. McKinsey and Patrick Suppes: Transformations of systems of classical particle mechanics.

This paper is a continuation of the investigation of the foundations of particle mechanics begun by McKinsey, Sugar, and Suppes (Bull. Amer. Math. Soc. Abstract 57-6-524). It is devoted to a determination of the set $S$ of transformations which always carry systems of particle mechanics into systems of particle mechanics. It is shown that every Galilean transformation belongs to $S$, as does every change of units (of time, mass, distance, and force). Moreover, every member of $S$ which satisfies the following weak hypothesis is expressible as a product of Galilean transformations and changes of units: the transformation of time is a one-to-one function mapping $R$ (the set of real numbers) onto itself; the transformation of mass is a function mapping the 
set of positive real numbers into itself; the transformation of position is a function mapping $E_{n} \times R$ (where $E_{n}$ is the set of all $n$-dimensional vectors) into $E_{n}$; and the transformation of force is a one-to-one function mapping $E_{n}$ onto itself. In general the units of distance and force can be transformed differently along the different axes; this is not the case, however, if the system is ultra-classical: i.e., if the forces satisfy Newton's Third Law and are expressible as functions only of the distances between the particles. (Received July 14, 1952.)

\section{Statistics and Probability}

\section{2t. K. L. Chung: On the renewal theorem in higher dimensions.}

Let $V_{1}, V_{2}, \cdots$ be independent $r$-dimensional random vectors with the common distribution $F\left(x_{1}, \cdots, x_{r}\right)$ which does not degenerate into a one-dimensional distribution. Let $m_{j}, j=1, \cdots, r$, be the mean of the $j$ th component. Let $S_{n}=\sum_{k=1}^{n} V_{k}$ and let $E\left(n \mid S_{n} \in B\right)$ denote the mathematical expectation of the number of $n$ 's such that $S_{n}$ assumes a value in the Borel set $B$ in $R^{r}$. Let $C$ be a compact set in $R^{r}$ and if $U$ is a vector in $R^{r}$ let $C(+) U$ denote the set obtained by a translation of $C$ through $U$. Let $|U|$ denote the length of $U$. Then, under the hypothesis that not all $m_{j}$ are zero, we have $\lim E\left(n \mid S_{n} \in C(+) U\right)=0$ as $|U| \rightarrow \infty$. (Received June 23, 1952.)

703. J. L. Hodges and Murray Rosenblatt: Recurrence-time moments in a Markov chain.

Consider an irreducible time-homogeneous Markov chain with discrete time. It is shown that if the recurrence time of one state has its first $k$ moments finite, then the recurrence times of all the other states have their first $k$ moments finite. The recurrence time moments of a class of random walks are examined. For $k=0,1,2, \cdots$ a random walk is exhibited whose first $k-1$ recurrence time moments exist and whose higher moments are infinite. A comparison theorem then permits the moment properties of other random walks to be determined. (Received April 10, 1952.)

704t. Miriam A. Lipschutz: Strong laws for sums of independent random variables with stable distributions.

Consider a sequence of identically distributed independent positive random variables such that $P(X \geqq x)=h(x) / x^{\alpha}(0<\alpha<2)$ and $\lim _{(x \rightarrow \infty)} h(c x) / h(x)=1$, for every positive constant $c$. Let $S_{n}=\sum_{k=1}^{n} X_{k}$. It is known that with $b_{n}$ properly chosen $P\left(S_{n} \leqq b_{n} x\right) \rightarrow G_{\alpha}(x)$ and $P\left(S_{n}-n p \leqq b_{n} x\right) \rightarrow G_{\alpha}(x)$ for $0<\alpha<1$ and $1<\alpha<2$ respectively, where $p$ denotes the first moment, and $G_{\alpha}(x)$ is the stable distribution function of index $\left(\alpha_{1}-1\right)$. Under those conditions where a satisfactory estimate of the error, in the above limit theorem, has been obtained, one gets the following strong lower bounds for $S_{n}$. Let $\psi(n) \uparrow \infty, \psi(n) \leqq 1 g n^{1 / 1-\alpha \mid}$, then $P\left(S_{n} \leqq b_{n} / \psi(n)^{1 / \alpha} \alpha_{\text {i.o. }}\right)$ and $P\left(S_{n}-n p \leqq-b_{n} \psi(n)^{1 / \alpha}\right.$ i.o. $)$ equal 0 or 1 as $\int\left[\psi(n)^{(1 / 2)(1 /(1-\alpha))} / n\right]$ exp $\left(-k_{\alpha} \psi(n)^{1 /(1-\alpha)}\right) d n$ and $\int \psi(n)^{(1 / 2)(1 /(\alpha-1))}\left(\exp \left(-k_{1 / \alpha} \psi(n)^{1 /(\alpha-1))}\right)\right.$ converge or diverge. $k_{\alpha}$ is a positive constant less than one. A recent lemma of Chung and Erdös (Trans. Amer. Math. Soc. vol. 72 (1952) p. 179) was used in the proof, which also allows one to obtain in a simple way the strong upper bounds previously discovered by Feller. (Trans. Amer. Math. Soc. vol. 67 (1949) p. 115). (Received May 22, 1952.)

705. Shu-Teh C. Moy: Characterizations of conditional expectation as a transformation on function spaces.

Let $T$ be a transformation on the space $S$ of all real non-negative measurable 
functions on a probability space $(\Omega, F, \mu)$ satisfying the following conditions: T1. $T(\alpha x+\beta y)=\alpha T x+\beta T y$, T2. If $x$ is bounded then $T x$ is bounded, T3. $T(x T y)$ $=(T x)(T y)$, T4. If $x_{n} \uparrow x$ then $T x_{n} \rightarrow T x$. It is proved that the general form of such transformation is to take $x$ to be the conditional expectation of $x g$ with respect to a $\sigma$-algebra of subsets $F_{T} \subset F$ where $F_{T}$ and $g$ are determined by $T$. With slight modification of the argument the same formulation is obtained for a continuous linear transformation $T$ on $L_{p}$ into $L_{p}$ satisfying conditions that $T$ takes bounded functions to bounded functions and that $T(x T y)=(T x)(T y)$ for bounded $x, y$. In the case of $p=1$, if $T$ satisfies the further conditions of $T 1=1$ and $\|x\|_{1}=\|T x\|_{1}$, where \|\|$_{1}$ denotes the $L_{1}$ norm, then it is proved that $T x$ is the conditional expectation of $x$ with respect to $F_{T}$. (Received July 14,1952 .)

\section{TOPOLOGY}

706. R. D. Anderson: Monotone mappings and monotone interior mappings of manifolds. Preliminary report.

The author shows that if $f$ is a monotone mapping of a compact $n$-manifold $S$ either with or without boundary and with $n>2$, then there exists a monotone interior mapping of $S$ onto $f(S)$. (Received July 21,1952 .)

\section{7t. H. W. Becker: Nonconnective topology. Preliminary report.}

A nonplanar dual has indeterminate connectivity, but has the same branch symmetry partition as the nonplanar itself. Represent such a topological imaginary by its real dual drawn in a color different from that used for reals. Assemble a graphoid (akin to the matroids of Hassler Whitney, Amer. J. Math. vol. 57 (1935) p. 509) from $m$ nonplanar circuits or their imaginary duals alike or different in any series-parallelbridge pattern. Then if $K_{m}$ is the number of combinations of $m$ objects of given specification, the possibilities are one real graph, one pure imaginary, and $K_{m-2}$ imaginary graphoids having imaginary duals, "dually imaginary graphoids," d.i.g. If the $m$ are all different, $K_{m}=2^{m}$; if all alike, $K_{m}=m+1$. Thus the reals are ultimately a drop in the bucket, among the nonconnectives. Using symmetry diversity methods previously applied to the enumeration of real graphs and passive circuits, for $16 \leqq n \leqq 19$ the d.i.g. $\langle a\rangle_{n}=1,18,238,2730$, and the imbedded passive circoids $\langle b\rangle_{n-1}=4,110,1912$, 26586; $\langle a\rangle_{16}$ is self-dual. Tentatively, $\langle b\rangle_{n} \sim 8 \cdot 15^{n-15}$. The natural habitat of Starr's theorem $\partial_{z} Z(z, \cdots)=\square$ is then $s_{n}+|b|_{n}+2(b)_{n}+\langle b\rangle_{n}$, the planar passive circuits, the nonplanar and their imaginary duals, and the d.i.p.c. (for which last, the Kuratowski's theorem analogue is that each contains one or more of the three elementary n.p.c. and one or more of the three duals of same). These are issues raised by R. M. Cohn. (Received July 21, 1952.)

\section{Arthur Bernhart: $A$ triangle inequality in coloring rings.}

The occurrence of a polygon adjacent to three regions of an $n$-ring yields inequalities among three associated frequencies in its 4-color spectrum. These requirements permit arguing from the algebraic spectrum to the geometrical structure. This triangle rule is illustrated on various 6-ring configurations. (Received July 21,1952.)

709. C. E. Burgess: Continua which are the sum of a finite number of indecomposable continua.

A continuum $M$ is said to be the finished sum of the continua of a collection $G$ if 
$M$ is the sum of the continua of $G$ and no continuum of $G$ is a subset of the sum of the others. A continuum $M$ is said to be indecomposable under index $n$ if $M$ is the finished sum of $n$ continua and is not the finished sum of $n+1$ continua. These two definitions were given by Swingle (Generalized indecomposable continua, Amer. J. Math. vol. 52 (1930) pp. 647-658). The following theorems are proved. If $n>1$ and $M$ is a compact continuum in a space satisfying R. L. Moore's Axioms 0 and 1 , then in order that $M$ be indecomposable under index $n$, it is necessary and sufficient that $M$ should be the finished sum of $n$ indecomposable continua and be irreducible about some $n$ points. If the compact continuum $M$ in the plane is the finished sum of two indecomposable continua $H$ and $K$ such that some composant of $H$ does not intersect $K$, then uncountably many composants of $K$ lie in $M-H$. (Received July 16, 1952.)

\section{D. O. Ellis: Lattice theory problem 77.}

Problem 77 of G. Birkhoff's Lattice theory is answered in the affirmative. One first shows by metric methods that it is valid for $2^{B}$ where $B$ is a countable set, by algebraic methods from validity in $2^{B}$ that it is valid in $2^{S}$ where $S$ is any set, again by algebraic methods that it is valid in $2^{S} / J$ where $J$ is a $\sigma$-ideal of $2^{S}$, and an application of the Theorem of Loomis then yields the result for any $\sigma$-complete Boolean algebra. (Received June 30, 1952.)

\section{Mary E. Estill: Problems on indecomposable connexes and related sets in the plane.}

The purpose of this paper is to answer the questions raised by P. M. Swingle in a paper entitled The closure of types of connected sets which appeared in the April, 1951 issue of the Proceedings of the American Mathematical Society. The principal results are the following. If $I$ is an indecomposable connexe in the plane, there does not exist a point $p$ such that $I+p$ is decomposable. If $W$ is a widely connected set in the plane, there always exists a point $q$ such that $W+q$ is widely connected. There is a biconnected set without a dispersion point which is not widely connected and is therefore not contained in a widely connected set. (Received July 7, 1952.)

\section{2t. M. K. Fort: A theorem concerning various types of weak con-} tinuity.

Let $T_{1}$ be a separable metric topology for a set $X$. A second topology $T_{2}$ for $X$ is $\alpha$-related to $T_{1}$ if and only if: there exists a countable collection of ordered pairs $\left(U_{n}, K_{n}\right)$ of subsets of $X$ such that $U_{n} \in T_{1}$ for each $n$, if $p \in U \in T_{1}$ then there exists $n$ such that $p \in U_{n} \subset K_{n} \subset U$, if $q \in U_{n}$ for some $n$ then there exists $V \in T_{2}$ such that $q \in V$ and $V-K_{n} \in T_{2}$. (In most examples $K_{n}=\bar{U}_{n}$.) It is proved that a function on a topological space into $X$ which is $T_{2}$ continuous is also $T_{1}$ continuous except at points of a first category subset of the domain. The following examples are typical: the pointopen topology is $\alpha$-related to the compact-open topology for the function space of continuous functions on a locally compact separable metric space into a separable metric space, the "weak neighborhood topology" in the calculus of variations sense is $\alpha$-related to the "strong neighborhood topology," the weak topology for a separable Banach space is $\alpha$-related to the norm topology. The theory developed has application in various fields, including: topological groups, transformation groups, measure theory, and semi-continuity. (Received April 9, 1952.) 
713. S. I. Goldberg: Extensions of Lie algebras and the third cohomology group. I.

Let $\{W, Q\}$ be a representation module of the Lie algebra $L$ and $U$ an $L$-module extending the $L$-module $W$ by the $L$-module $V$. Hence the extension $R_{x}(x \in L)$ of $Q_{x}$ to $U$ is a linear endomorphism of $U$ and $\{U, R\}$ is a representation module of $L$. The quotient $U / W$ implies $\{V, P\}$. Choose linear representatives $\mu_{v} \in U(v \in V)$ from the residue class corresponding to $v$ by $U / W \cong V$. Then $R_{x} \mu_{v}=\mu_{p_{x} v}+\beta(x, v)$, $\beta(x, v) \in W$. The factor system $\{\beta\}$ completely determines the structure of $U$, and so we write $U=(L, V, W, \beta)$. Given the extension $(L, V, W, \beta)$ there exists an exact relative cohomology sequence of homomorphisms, $\cdots \rightarrow H^{q}(L, V) \rightarrow^{\Delta} H^{q+1}(L, W)$ $\rightarrow{ }^{M} H^{a+1}(L, U) \rightarrow^{N} H^{a+1}(L, V) \rightarrow H^{q+2}(L, W) \rightarrow \cdots$. Let $\psi: U \rightarrow V$ be the given homomorphism of $U$ upon its quotient, and let $g$ be any cocycle in $C q(L, V)$. Pick representatives $\bar{g}\left(x_{1}, \cdots, x_{q}\right)$ at random so that $\bar{g}$ is multilinear and $\psi \bar{g}\left(x_{1}, \cdots, x_{q}\right)$ $=g\left(x_{1}, \ldots, x_{q}\right)$. The map $\Lambda$ is obtained by sending the cohomology class of $g$ in $H^{q}(L, V)$ into that of $\delta \bar{g}$ in $H^{q+1}(L, W)$. The following is established: Given the representation module $\{W, Q\}$ then, for any given extensions $L^{*}=(L, V, g)$ and $U$ $=(L, V, W, \beta)$, a necessary and sufficient condition for the existence of another extension $L^{* *}$ of $L$ by $U$ with $L^{* *} / W \cong L^{*}$ is that $\Lambda(g)$ be a coboundary. (Received July 9, 1952.)

714t. S. I. Goldberg: Extensions of Lie algebras and the third cohomology group. II.

Let $L$ be a Lie algebra over an arbitrary field and $\{X, S\}$ a representation module of $L$. Then for any given extension $U^{*}=(L, U, X, \gamma)$, there exist extensions $W^{*}$ $=(L, W, X, \gamma)$ and $U=(L, V, W, \beta)\left(W^{*}\right.$ is a submodule of $U^{*}$ and $\left.U / W \cong V\right)$. Let $L^{*}=(L, V, g)$ and $L^{* *}=(L, U, \bar{g}+\alpha)$ satisfying $L^{* *} / W \cong L^{*}$. Select linear representatives $\sigma_{x}, \sigma_{y}, \cdots \in L^{* *}$. Hence $\left[\sigma_{x}, \sigma_{y}\right]=\sigma_{[x, y]}+\bar{g}(x, y)+\alpha(x, y), \alpha \in C^{2}(L, W)$. A necessary and sufficient condition for the existence of the extension $L^{* * *}=\left(L, U^{*}\right)$ with $L^{* * *} / X \cong L^{* * *}$ is that the $3-S$-cocycle $\bar{\Lambda}(\bar{g}+\alpha)$ is a coboundary $(\bar{\Lambda}$ is the invariant coboundary, $\left.\bar{\Lambda}: H^{q}(L, U) \rightarrow H^{q+1}(L, X)\right)$. (Received July 9, 1952.)

\section{5t. Mary-Elizabeth Hamstrom: Concerning webs in the plane.}

The terms "web" and "simple web" have been defined by R. L. Moore. (Cf. R. L. Moore, Concerning continua which have dendratomic subsets, Proc. Nat. Acad. Sci. U. S. A. vol. 29 (1943) pp. 384-389, and A characterization of a simple plane web, Proc. Nat. Acad. Sci. U. S. A. vol. 32 (1946) pp. 311-316.) In the present paper the notion of a $W_{n}$ set is introduced. If $n>1$, a $W_{n}$ set is a compact continuum $M$ for which there exists a family $F$ of $n$ elements such that (1) each element $o$ of $F$ is an upper semicontinuous collection of mutually exclusive continua which fills up $M$ and is an arc with respect to its elements and (2) if $G$ is a collection of continua each belonging to some, but no two to the same, collection of the family $F$, then the continua of the collection $G$ have a point in common and their common part is totally disconnected. It is shown that there exists a $W_{2}$ set which is not a $W_{3}$ set and that for each positive integer $n$ a simple closed curve plus its interior is a $W_{n}$ set. (Received June 23, 1952.)

\section{6t. Mary-Elizabeth Hamstrom: Concerning a certain type of web.}

A $W_{n}^{\prime}$ set is a $W_{n}$ set satisfying the conditions obtained by replacing, in the definition of a $W_{n}$ set (see abstract 58-6-715) the phrase "upper semicontinuous collection 
of mutually exclusive continua" by the phrase "continuous collection of mutually exclusive continuous curves." If $M$ is a compact continuum in the plane, $B(M)$ will denote its boundary and $S(M)$ will denote the collection consisting of all boundaries of complementary domains of $M$, all points of $B(M)$ that are not on the boundary of any complementary domain of $M$, and all points of $B(M)$ that are common to the boundaries of two or more complementary domains of $M$. It is shown that the compact continuum $M$ in the plane is a $W_{2}^{\prime}$ set if and only if (1) every boundary of a complementary domain of $M$ is a simple closed curve and (2) the collection $S(M)$ is an upper semicontinuous collection of type 2 (cf. R. L. Moore, Fundamental theorems concerning point sets, The Rice Institute Pamphlet, vol. 23, 1936, pp. 42, 43, and 56) and there exists an upper semicontinuous collection, $S^{\prime}$, of type 2 such that $S(M)$ is a subcollection of $S^{\prime}$, each element of $S^{\prime}-S(M)$ is a point of $M-B(M), S^{\prime}$ is an arc with respect to its elements (cf. R. L. Moore, loc. cit. p. 2) and if $J$ is the boundary of a complementary domain of $M$ there are only two points $P$ of $J$ such that $P$ is a limit point of the sum of the elements of $S^{\prime}$ different from $J$. If $n$ is an integer greater than 2 the theorem remains true if " $W_{2}^{\prime}$ " is replaced by " $W_{n}^{\prime}$." (Received June 23, 1952.)

\section{Frank Harary: The number of graphs and directed graphs.}

The powerful and elegant methods of Pólya, Acta Math. (1937), yield formulas for the number of graphs, directed graphs, and several generalizations of graphs. The generalizations considered are graphs of type $t$, graphs of strength $s$, and multiply rooted graphs. A graph of strength $s$ is one in which there are at most $s$ lines between any pair of points. A graph of type $t$ has $t$ different kinds of lines and at most $t$ lines (each of a different kind) joining any pair of points. A multiply rooted graph is one in which the set of points is partitioned into distinguished classes. The formula for the number of graphs is due to Polya; graphs of strength $s$ were counted by R. Z. Norman. To find the number of graphs of $n$ points and $k$ lines, a polynomial $G_{n}(x)$ of degree $n(n-1) / 2$ is determined in which the coefficient of $x^{k}$ is this number. Directed graphs, graphs of type $t$, and of strength $s$ are counted by suitable changes in the "figure power series" (P6lya's terminology). Multiply rooted graphs of $n$ points are found by using as the appropriate permutation groups the direct product of symmetric groups having $n$ as the sum of their degrees. (Received June 13,1952.)

\section{8t. S. T. Hu: Cohomology relations in spaces with a topological transformation group.}

Let $Q$ be a topological transformation group operating on the left of a topological space $X$. Let us denote by $B$ the orbit space and $p: X \rightarrow B$ the projection. $p$ is a continuous and open map of $X$ onto $B$. For any abelian coefficient group $G$, the continuous map $p$ induces homomorphisms $p^{*}: H^{n}(B, G) \rightarrow H^{n}(X, G)$ of the Alexander-Wallace cohomology groups. These induced homorphisms are, in general, not onto isomorphisms. They depend on the manner in which the topological transformation group $Q$ operates on $X$. To measure the deviation of these induced homomorphisms $p^{*}$ from the onto isomorphisms, the author introduces, in the present paper, the weakly residual cohomology groups $H_{w}^{n}(X, G)(n \geqq 0)$. They are invariants depending on $X, Q, G$ and the operations of $Q$ on $X$. By means of these groups, the author establishes an exact sequence $H^{0}(B, G) \rightarrow p^{*} \ldots \rightarrow H^{n}(B, G) \rightarrow p^{*} H^{n}(X, G) \rightarrow H_{v w}^{n}(X, G)$ $\rightarrow H^{n+1}(B, G) \rightarrow p^{*} \ldots$. This indicates that the weakly residual cohomology groups $H_{v}^{n}(X, G)$ might play an important role in the further studies of the cohomology structures of the orbit space. For each point $x$ of $X$, there is a canonical homomorphism 
$k_{x}^{*}$ of $H_{w}^{n}(X, G)$ into $H^{n}(Q, G)$ for each $n \geqq 0$. It is proved that if $Q$ is compact and if $x$ and $y$ are two points contained in a compact connected subset of $X$ then $k_{x}^{*}=k_{y}^{*}$. (Received July 9, 1952.)

\section{9t. E. G. Kundert: A generalisation of the fundamental group.}

The following generalization of the fundamental group to the second dimension seems to be a natural one: let $\left(T_{h}, f\right)$ be the continuous mapping $f$ of the carrier $\left(T_{h} ; p\right)$ into the topological space $\left(Y ; y_{0}\right)$ such that $p \rightarrow y_{0}, T_{h}$ being an orientable, ordered (handles ordered) 2-dimensional manifold with $h$ handles. By certain continuity preserving, reversible processes $\left(T_{h}, f\right) \leftrightarrow\left(T_{h^{\prime}}, f^{\prime}\right)$ and the usual homotopy relation, one is able to collect the $\left(T_{h}, f\right)$ in equivalence classes, which form a group $\Pi(Y)$. Some properties: $\Pi=\Pi_{1} \times \Pi_{2}$ such that $\Pi_{1} \approx \pi_{1}$ (fundamental group). $\pi_{2}$ (homotopy group) is subgroup of $\Pi_{2} \cdot \Pi_{2} \rightarrow \Gamma H_{2} ; \gamma=\Gamma \bigcap \pi_{2} ; \pi_{2} \rightarrow{ }^{\gamma} S_{2}, \Pi_{2} / \Gamma \cdot \pi_{2} \approx G_{1}^{*}$. If $\pi_{1} \approx 0: \Pi_{2}$ $\approx \pi_{2}$. (For the definitions of $S_{2}$ and $G_{1}^{*}$ see H. Hopf, Fundamental Gruppe und zweite bettische Gruppe, Comment. Math. Helv. vol. 14.) (Received August 11, 1952.)

720t. Deane Montgomery and Leo Zippin: Small subgroups of finite-dimensional groups.

The authors prove the following "reduction-theorem": Theorem A. If $G$ is a separable metric, locally compact, finite-dimensional, connected, and locally connected topological group and if all of the proper subgroups of $G$ are generalized Lie groups (in the sense of Gleason, or of Iwasawa) then $G$ contains an invariant generalized Lie group $H$ such that the factor-group $G / H$ is finite-dimensional and has no small subgroups. This means that there is a neighborhood of the identity of $G / H$ which contains no subgroup of $G / H$ except the trivial one consisting of the identity alone. The subgroup $H$ is determined in a natural way so that the group $G / H$ shall have no center but the identity. A key device in the proof is that of finding a local Lie subgroup $R$ of $G / H$ such that $R$ is equal, in the small, to the identity component of its own normalizor and such that any conjugate subgroup $R^{\prime}$ to $R$ either coincides with $R$, locally, or else intersects $R$ in the identity alone. Such a subgroup, under conjugation by the elements of a neighborhood of $G / H$, sweeps out some open subset of $G / H$. This gives one control of the topology of $G / H$ and also of the topological space which results when $G / H$ is fibered by $R$. (Received March 27, 1952.)

\section{1t. J. C. Moore: On tetrads and triads.}

The triad $(X ; A, B)$ is said to be excisive if and only if $X=A \cup_{B}, A, B$, and $A \cap B$ are arcwise connected, and $H_{q}(X, A)=H_{q}(B, A \cap B)$ for all $q$, the homology theory considered being singular homology. It is proved that if $(X ; A, B, C)$ is a tetrad such that the triads $(X ; A, B),(X ; A, C),(X ; B, C)$, and $(A ; A \cap B, A \cap C)$ are excisive, $X$ is 1-connected, $(X, A),(A, A \cap B)$, and $(A, A \cap C)$ are 2-connected, $R$ is a principal ideal domain, $H_{q}(X, A ; R)=0$ for $q \leqq r, H_{q}(X, B ; R)=0$ for $q \leqq m$, and $H_{q}(X, C ; R)$ $=0$ for $q \leqq n$, then $\pi_{q}(X ; A, B, C) \otimes R=0$ for $q \leqq m+n+r$. The preceding theorem is applied to prove that if $(X ; A, B)$ is an excisive triad, $X$ is 1 -connected, $C^{*}$ is the join of $A \cap B$ and a point, $A^{*}=A \cup C^{*}, B^{*}=B \cup C^{*}$, and $X^{*}=A^{*} \cup B^{*},(X, A)$, $(A, C)$, and $(B, C)$ are 2-connected, $R$ is a principal ideal domain, $H_{q}(C ; R)=0$ for $0<q<r, H_{q}(X, A ; R)=0$ for $q \leqq m$, and $H_{q}(X, B ; R)=0$ for $q \leqq n$, then $\pi_{q}(X ; A, B) \otimes R$ $=\pi_{q}\left(X^{*} ; A^{*}, B^{*}\right) \otimes R$ for $q<m+n+r$, and $\pi_{m+n+r}(X ; A, B) \otimes R \rightarrow \pi_{m+n+r}\left(X^{*} ; A^{*}, B^{*}\right)$ $\otimes R \rightarrow 0$ is exact, where the homomorphisms are those induced by the inclusion map. Since $C^{*}=A^{*} \bigcap B^{*}$ is contractible, $\bigvee \pi_{q}\left(X^{*} ; A^{*}, B^{*}\right)=\pi_{q+1}\left(A^{*} \times B^{*}, A^{*} V B^{*}\right)$ for all $q$. (Received June 6, 1952.) 


\section{J. C. Moore: On the homotopy groups of odd-dimensional} spheres.

For $G$ an abelian group and $p$ a prime let $C(G, p)$ denote the $p$-primary component of $G$. It is then proved that for $p$ an odd prime, $C\left(\pi_{q}\left(S^{3}\right), p\right)$ is a cyclic group of order $p$ for $q=4 p-3,4 p-2$, and is trivial for $4 p-2<q<6 p-6$. Let $F_{2}^{n}$ denote the space of two cells in $S^{n}$. Then in a standard fashion $S^{n}$ may be imbedded in $F_{2}^{n+2}$ so that the inclusion map is equivalent to the double suspension. Let $X_{p}^{*}$ denote the space obtained by adjoining an $r+1$ cell to the $r$ sphere by a map of degree $p$. It is proved that if $n$ is odd and greater than or equal to 3 , then $C\left(\pi_{q}\left(F_{2}^{n+2}, S^{n}\right), p\right)=\pi_{q}\left(X_{p}^{p n+p-2}\right)$ for $q$ less than $(p+1)(n+1)-4$. Moreover $C\left(\pi_{q}\left(F_{6}{ }^{6} S^{3}\right)\right)$ is a cyclic group of order $p$ for $q=4 p-2$, and is trivial otherwise for $q$ less than $6 p-5$. These facts imply that if $n$ is odd and greater than 3 , then $C\left(\pi_{q}\left(S^{n}\right), p\right)$ is nontrivial for $q=n+4 p-5$, and is trivial for $n+4 p-5<q<n+6 p-9$. (Received June 26, 1952.)

\section{P. S. Mostert: On the existence of local cross sections in compact} groups.

Let $G$ be a second countable topological group, $H$ a subgroup of $G$. Theorem. If $G$ has a discrete normal subgroup $N$ such that $G / N$ is compact and of finite dimension, then $H$ has a local cross section. Moreover, if $G$ is 0 -dimensional compact, then $H$ has a full cross section. $G$ (compact) is the projective limit of Lie groups $G_{k}$. Local cross sections are constructed for $H_{k} \subset G_{k}$, where $H_{k}$ is the projection of $H$ on $G_{k}$, which "agree" under the projections. (Received July 21, 1952.)

724. Everett Pitcher: Homology theory of critical levels for a pair of spaces. Preliminary report.

A homology theory of critical levels of a real-valued function $f$ on a space $X$ relative to a subspace $Y$ is constructed. The theory is formulated in terms of group homomorphisms. It connects the Morse critical level theories for $f$ on $X$ and for $f \mid Y$ through interlocking exact sequences. (Received July 21,1952.)

725. L. E. Pursell: Rings associated with topological spaces. Preliminary report.

A ring $R$ is "weakly associated" with a $T_{1}$-space $X$ if, for every $r$ in $R$, there is a closed set $Z(r)$ in $X$ such that: (i) $r=0$ if and only if $Z(r)=X$, (ii) $r s=0$ if and only if $Z(r) \cup Z(s)=X$, and (iii) if the point $x$ is not in a closed set $F$, there is an $r$ such that $F \subset Z(r)$ but $x \notin Z(r)$. The "support" $S(r)$ is the set $\mathrm{Cl}(X-Z(r))$. In this ring $r s=0$ implies $s r=0$. The "annihilator" $A(r)$ is the ideal of all $s$ such that $r s=s r=0$. Elements $r$ and $r^{\prime}$ in $R$ are "disjunct" if, for every $s$ and $s^{\prime}$ in $R, \exists t \in R$ such that $(t-s) \in A(r)$ and $\left(t-s^{\prime}\right) \in A\left(r^{\prime}\right)$. A ring $R$ weakly associated with $X$ is "associated with $X^{\prime}$ " if (iv) $r$ and $r^{\prime}$ in $R$ are disjunct if and only if $S(r)$ and $S\left(r^{\prime}\right)$ are disjoint and (v) $x \notin$ closed set $F$ implies $\exists r \in R$ such that $F \subset S(r)$ and $x \notin S(r)$. If condition (iv) is satisfied, if every $S(r)$ is compact, and if (v) is satisfied for $F$ compact, then $R$ is "compact associated" with $X$. A ring associated with a compact Hausdorff space determines its topology. A ring compact associated with a regular locally-compact Hausdorf space determines its topology. The ring of all continuous functions on a normal $T_{1}$-space is associated with the space. The ring of such functions with compact supports is compact associated with the space. Rings can be constructed of tensors on differentiable manifolds which are associated or compact associated with the manifold. (Received July 18, 1952.) 
726. M. E. Shanks and L. E. Pursell: The Lie algebra of a smooth manifold.

Let $M$ be an infinitely differentiable manifold and $D$ the algebra of real infinitely differentiable functions on $M$. A tangent vector $T$ is an abstract derivative on $D$ and $T \cdot f=\tau^{i} \partial f / \partial x^{i}$, where $\tau^{i}$ are infinitely differentiable. The set $\mathcal{L}$ of all tangent vectors is a Lie algebra over the real field under the composition $\left[T_{1}, T_{2}\right] \cdot f=T_{1} \cdot\left(T_{2} \cdot f\right)$ $-T_{2} \cdot\left(T_{1} \cdot f\right)$. Let $\mathcal{L}_{0}$ be the subalgebra of $\mathcal{L}$ with compact supports. It is shown that two manifolds with isomorphic Lie algebras $\mathcal{L}_{0}$ are homeomorphic. Furthermore if $G$ is the Grassmann algebra of differential forms over $M$ and $G_{p}$ is the subalgebra of $G$ consisting of forms of degree $p$ and higher, $p<n$, then the Lie algebra of $M$ is determined by the algebraic structure of $G_{p}$. (Received July 21, 1952.)

\section{7t. Henry Sharp: Strongly topological imbedding of F-sigma subsets of $E_{n}$.}

Denote by $E_{n}$ Euclidean space of $n$ dimensions, by $M_{n}^{k}$ the set of points in $E_{n}$ having at most $k$ rational coordinates, by $L_{n}^{k}$ the set of points in $E_{n}$ having at least $k$ rational coordinates. It is an open question whether $M_{n}^{k}(n<2 k+1)$ contains a topological image of every $k$-dimensional subset of $E_{n}$. For the special cases $k=n$ and $k=n-1$ the answer to this question is in the affirmative; in fact, for these values of $k$ the set $M_{n}^{k}$ contains a strongly topological image (image under a homeomorphism of the space onto itself) of every $k$-dimensional subset of $E_{n}$. The present paper is concerned with a related problem: does the set $M_{n}^{k}$ contain a strongly topological image of every $j$-dimensional $F_{\sigma}$-subset of $E_{n}$. S. W. Hahn has given the answer to this problem for certain special cases, namely: affirmative for $n=2, j=k=0$, and $n=3$, $j=0, k=1$; negative for $n=3, j=k=0$. The solution of this problem is completed in the present paper in which it is proved that the answer is affirmative for $n \geqq 4, j \leqq k$ $=n-2$, and negative in all other (previously unsolved) cases. (Received June 4, 1952.)

\section{8t. A. H. Stone: $A$ note on infinitely multicoherent spaces.}

Eilenberg (Fund. Math. vol. 27 (1936) pp. 153-190) has defined the "degree of multicoherence," $r(S)$, of a connected topological space $S$ to be sup $b_{0}(A \cap B)$, the supremum being taken over all pairs of closed connected sets $A, B$ such that $A \cup B=S$. Here $b_{0}(X)$ denotes the number of components of $X$ less than one (or $\infty$ if this number is infinite). Assume that $S$ is a Peano space and that $r(S)=\infty$; need $A, B$ exist as above with $b_{0}(A \cap B)=\infty$ ? It is shown that the answer is affirmative if $S$ is 1-dimensional, but negative for some 2-dimensional Peano spaces. (Received May 28, 1952.)

\section{9t. A. H. Stone: On coverings of 2-dimensional spaces.}

The "density" of a normal space $S$ is the least integer $n$ such that $S$ has arbitrarily fine finite open (or, equivalently, closed) coverings in which no set meets more than $n$ others. Boltyanskii (Doklady Akad. Nauk SSSR vol. 75 (1950) pp. 605-608) has shown that if $S$ is a 2-dimensional compactum its density is at least 6 . The present paper shows that if $S$ is 2-dimensional its density is at most 7, and that the value 7 does occurs for suitable $S$. (Received May 28, 1952.)

730t. D. D. Strebe: Certain applications of irreducibility to connected Hausdorff spaces.

A study is made of Hausdorff spaces which are irreducibly connected or irre- 
ducibly closed and connected about arbitrary subsets. The nature of spaces which are irreducibly connected about minimal subsets (basic sets $B$ ) is investigated. If a nondegenerate connected space $M$ has a basic set $B$ consisting of $n$ points, where $n$ is a positive integer, it is proved that $M$ can be expressed as the sum of $n-1$ or less subsets, each of which is irreducibly connected about 2 points of $B$, and such that each point of $B$ is a point of irreducibility of at least one of these subsets. With regard to minimal subsets (basic sets $B^{*}$ ) about which a space $M$ is irreducibly closed and connected, it is proved that $B^{*}$, when it exists, is an isolated, nondense subset of $M$, consisting entirely of non-cut points. The existence of $B^{*}$ implies the existence of a basic set $B$. If a locally compact metric space $M$ has a basic set $B^{*}$, a necessary and sufficient condition that $B^{*}$ be the unique basic set is that $M$ be locally connected at each point of $B^{*}$. (Received July 14, 1952.)

731. Harold Tinnappel: On the topological index. Preliminary report.

Utilizing a theorem of T. Rad6 (Length and area, Amer. Math. Soc. Colloquium Publications, vol. 30, IV.1.71) and certain auxiliary transformations the following theorem is proved. If $T: z=f(w)$ is a continuous transformation from a bounded, simply-connected Jordan region $R$ in the $w$-plane into the $z$-plane and if for a point $z_{0}$ we have the topological index equal to $k \neq 0$, then there exists a $\delta>0$ such that there are at least $|k|$ maximal model continua lying in $D$ for each $z$ for which $0<\left|z-z_{0}\right|<\delta$, where $D$ is the component of $R-f^{-1}\left(z_{0}\right)$ which contains the boundary of $R$. An analogous theorem is proved for the case where $R$ is a bounded, finitely connected Jordan region. (Received July 16, 1952.)

\section{2t. A. D. Wallace: An axiom for cohomology.}

In this note we consider the effect of replacing (in a cohomology theory) the homotopy postulate by the following postulate: If $(Y, B)$ is an adequate pair and $h \in H^{n}(Y, B)$, then there is an open cover $\mathcal{V}$ of $Y$ such that if $f, g:(X, A) \rightarrow(Y, B)$ are adequate maps satisfying $x \in X$ implies the existence of a $V \in \mathcal{V}_{\text {with }} f(x), g(x) \in V$, then $f^{*}(h)=g^{*}(h)$. This is known to hold for the Alexander-Kolmogoroff groups (Spanier, Ann. of Math. (1948)). There are various modifications obtainable by prefixing an adjective to "open cover." This adjective depends on the particular type of "nearness" of the functions $f$ and $g$ that seems suitable for the problem at hand. If the spaces in question are metric then the postulate takes the familiar form: If $h \in H^{n}(Y, B)$, then there is an $\epsilon(h)>0$ such that $\rho(f, g)<\epsilon(h)$ implies $f^{*}(h)=g^{*}(h)$. (Received August 11, 1952.)

\section{3t. A. D. Wallace: $A$ note on mobs. I.}

A mob is such a map (=continuous function) $m: S \times S \rightarrow S$ that $S$ is Hausdorff and $m$ is associative. Write $x y=m(x, y)$ and say that $S$ is a mob. If $S$ has a unit and is compact, then the set $I$ of inverses is a compact (topological) group. If $S$ is also connected, then no element of $I$ is a cutpoint of $S$. If $I$ is compact it is a group, and if also $S$ is connected no point of $I$ cuts $I$ in $S$. (Received July 31, 1952.)

\section{4t. A. D. Wallace: $A$ note on mobs. II.}

Let $S$ be a mob (preceding abstract), let $E$ be its idempotents and $K$ its (unique) minimal closed ideal if such exists. Each algebraic subgroup is contained in a maximal such and these are pairwise disjoint. If $S$ is compact, each such is a compact (topo- 
logical) group. If $S$ is compact, then $K$ exists. If $e \in E$, let $H(e)$ be the maximal subgroup containing $e$. Let $S$ be compact. Then $H(e)=e S e$ if and only if $e \in K \cap E$ and $K=\bigcup\{H(e) \mid e \in K \cap E\}$. This topologizes algebraic results of Clifford-Miller, Green, Rees, Schwarz, and Suschkewitsch. As corollaries: (i) If $S$ has a unit and no other idempotent, it is a group; (ii) if $K$ has a unit, it is a group; (iii) If $S$ is simple and connected, then $S$ has no zero and $K=S$. If also $S$ has a unit, then $S$ is a group. (Received July $31,1952$.

\section{5t. G. T. Whyburn: $A$ unified space for mappings.}

In this paper it is shown that any mapping from one separable metric space to another is topologically equivalent to a partial mapping of a compact mapping (actually a retraction). This is accomplished by constructing a space unifying both the domain and the range spaces and showing that a compact mapping can be defined on this space which is topologically equivalent to the given one on the prototype of the original domain space. The unified space is separable and metrizable when the given spaces are locally compact, and it is in this setting that the new viewpoint and result seem to be most useful. (Received May 26, 1952.)

E. G. Begle, Secretary 